\title{
Cocaine-induced synaptic plasticity in the nucleus accumbens and drug-associated behavior - An unexpected dissociation
}

\author{
Dissertation \\ for the award of the degree \\ "Doctor of Philosophy (PhD)" \\ Division of Mathematics and Natural Sciences \\ of the Georg-August-Universität Göttingen \\ within the doctoral program IMPRS Molecular Biology \\ of the Georg-August University School of Science (GAUSS)
}

\author{
submitted by \\ Avani Shukla \\ from New Delhi, India
}

Göttingen, 2016 


\section{Thesis Committee}

Dr. Dr. Oliver Schlüter

Molecular Neurobiology, European Neuroscience Institute, Göttingen

Prof. Dr. Tobias Moser

Department of Otolaryngology, University Medical Center, Göttingen

Prof. Dr. Silvio Rizzoli

Department of Neuro- and Sensory Physiology, University Medical Center, Göttingen

\section{Members of the Examination Board}

Referee: Dr. Dr. Oliver Schlüter

Molecular Neurobiology, European Neuroscience Institute, Göttingen

$2^{\text {nd }}$ Referee: Prof. Dr. Tobias Moser

Department of Otolaryngology, University Medical Center, Göttingen

\section{Further members of the Examination Board}

Prof. Dr. Silvio Rizzoli

Department of Neuro- and Sensory Physiology, University Medical Center, Göttingen

Prof. Dr. Klaus-Armin Nave

Department of Neurogenetics, Max Planck Institute for Experimental Medicine, Göttingen

Prof. Dr. Ahmed Mansouri

Department of Clinical Neurophysiology, University of Göttingen

Prof. Dr. Mikael Simons

Department of Neurology, University of Göttingen

Date of oral examination: $10^{\text {th }}$ May 2016 
Herewith I declare that I prepared the PhD Thesis "Cocaine-induced synaptic plasticity in the nucleus accumbens and drug-associated behavior - An unexpected dissociation" on my own and with no other sources and aids than quoted.

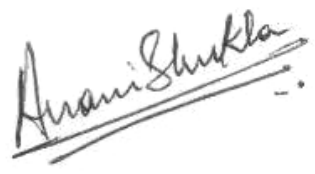

Göttingen, $21^{\text {st }}$ March 2016 
"Nothing in the world is worth having or worth doing unless it means effort, pain, difficulty........" 


\section{Table of contents}

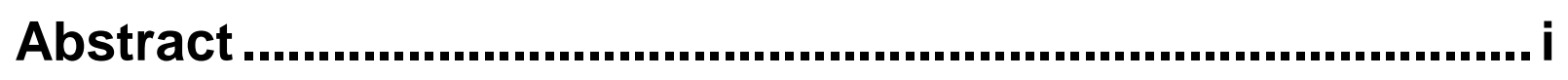

List of Figures ............................................................................ ii

\section{List of abbreviations}

\section{Introduction}

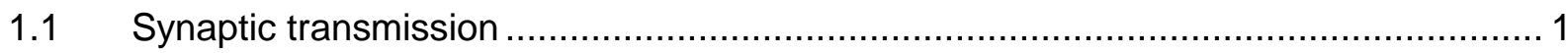

1.1.1 Presynaptic neurotransmitter release ....................................................... 2

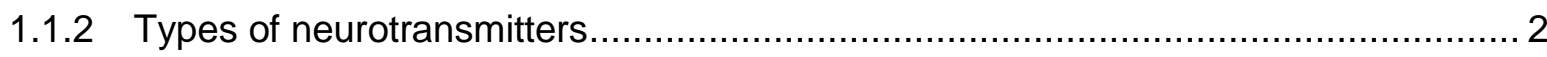



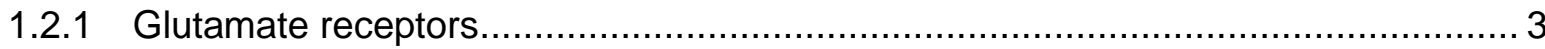

a) Ionotropic glutamate receptors - AMPARs, NMDARs and KARs.......................... 4

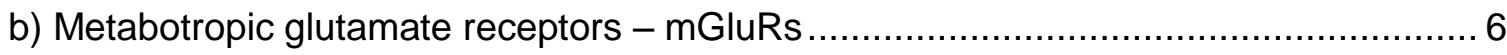

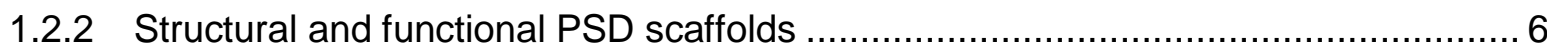

1.2.3 Synaptic plasticity - cellular basis of learning and memory …………………..... 7

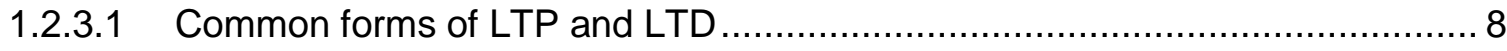

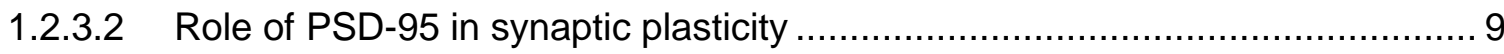

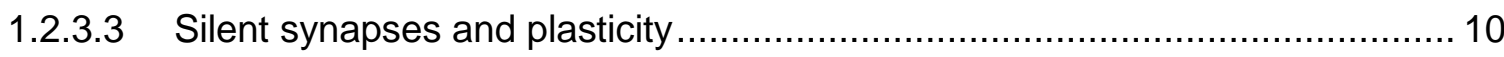



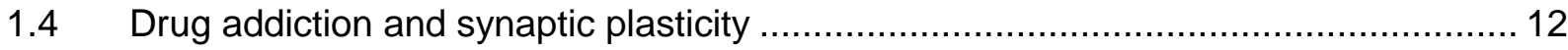

1.4.1 Behavioral tests for drug reinforcement in rodents .......................................... 13

1.4.2 Hijacking of the reward circuits of the brain ……........................................... 14

1.4.3 Cocaine-induced silent synapses in the adult brain ......................................... 16

1.4.4 CP-AMPAR expression and drug-related behavior......................................... 17

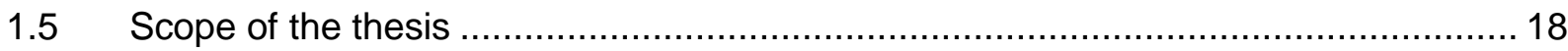




\section{Materials and Methods}

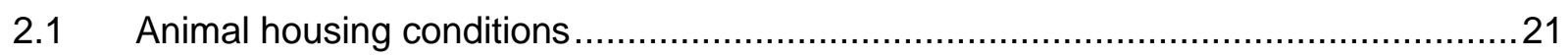

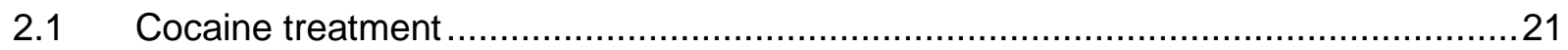

2.1.1 Passive injection of cocaine in mice .................................................. 21

2.1.2 Cocaine - Conditioned place preference (CPP) in mice ...................................21

2.1.3 Intravenous Self-Administration (SA) of cocaine in rats ...................................23

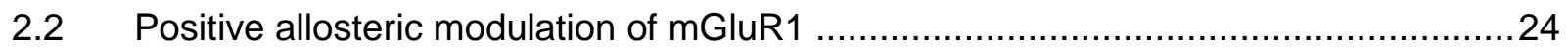

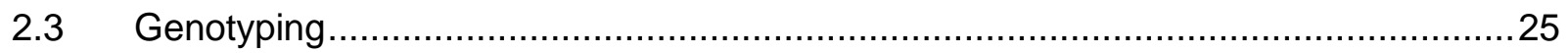

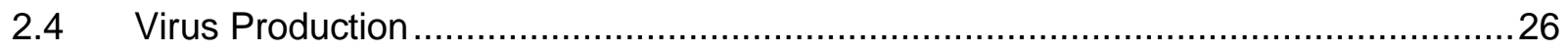



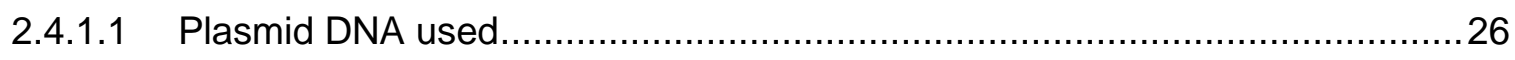

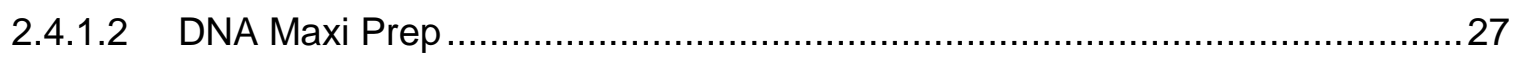

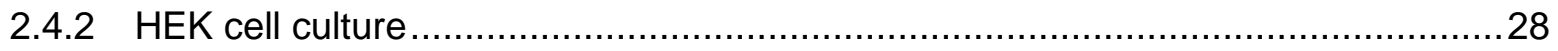

2.4.3 Adeno Associated Virus (AAV) production .................................................... 28

2.4.3.1 HEK 293T cell transfection for AAV8 preparation.......................................28

2.4.3.2 Harvesting and purification of AAV particles ............................................ 28

2.4.4 Extra large scale lentivirus preparation ....................................................29

2.4.4.1 HEK 293T cell transfection for lentivirus preparation ..................................29

2.4.4.2 Harvesting and purification of lentiviral particles..................................... 30

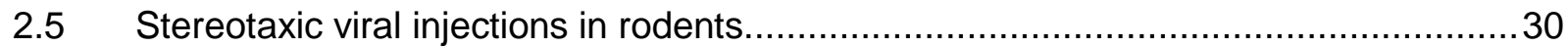

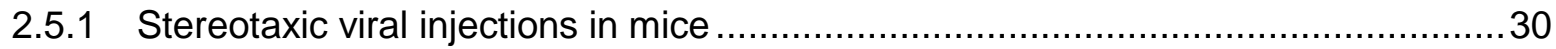

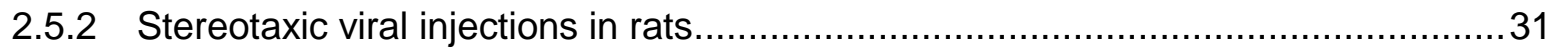

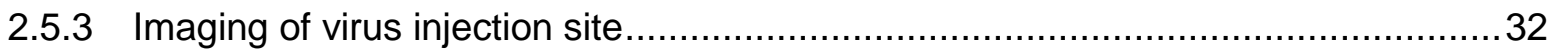

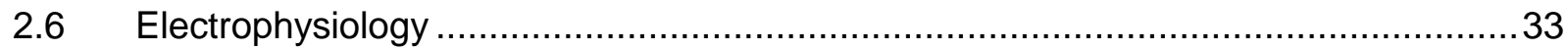

2.6.1 Preparation of acute brain slices of the NAc .................................................... 33

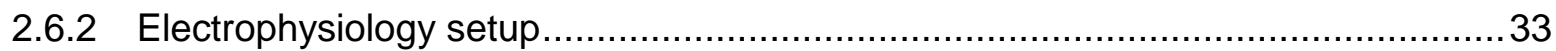

2.6.3 Electrophysiological recordings from the NAc shell.....................................35

2.6.3.1 Failure Analysis - Quantification of proportion of silent synapses ...................35

2.6.3.2 AMPAR Rectification Index - Quantification of proportion of CP-AMPARs ....36 


\section{Results}

3.1 Cocaine-conditioned place preference in wild type mice and long-term retention of drug-related memory

3.2 Increase in number of silent synapses in the nucleus accumbens shell upon cocaine exposure and accumulation of calcium permeable AMPARs during withdrawal from cocaine 42

3.2.1 The positive association learnt during cocaine-CPP and withdrawal from cocaine is necessary for accumulation of CP-AMPARs into the NAcSh synapses 45

3.3 mGluR1 activation re-silences NAcSh synapses by removal of CP-AMPARs..... 47

3.4 PSD-95 KO mice learn the cocaine-CPP association but do NOT retain it after prolonged withdrawal. The NAcSh has a high proportion of silent synapses that do NOT mature to CP-AMPAR containing synapses

3.5 SAP102 KO mice learn and remember the cocaine-CPP association even though the NAcSh has a high number of silent synapses lacking CP-AMPARs 53

3.6 NAcSh-restricted knock-down of PSD-95 does not impair retention of drug-associated memory in WT or SAP102 KO mice 57

3.7 PSD-95 and/or SAP102 are required in the NAcSh MSNs for maturation of silent synapses

3.8 NAcSh-restricted knock down of PSD-95 in rats, does not affect cocaine selfadministration 63

3.8.1 NAcSh-restricted knock down of PSD-95 in rats, does not impair incubation of cocaine craving. 66

3.9 Cocaine induces silent synapses via the thrombospondin receptor $\alpha 2 \delta 1$ 69 


\section{Discussion}

4.1 The association learnt by the mice in the cocaine-CPP paradigm becomes a robust long-term memory

4.2 Cocaine induces silent synapses in the NAcSh which mature during withdrawal from cocaine

4.3 Silent synapses of the NAcSh mature into CP-AMPAR containing synapses, when animals learns to associate environmental cues to the rewarding experience of cocaine......75

4.4 MAGUKs are essential for maturation of silent synapses but not for retention of drugassociated memory.

4.5 PSD-95 in the NAcSh is unlikely to be required for incubation of cocaine craving .....80

4.6 Long-term retention of drug-associated memory may be coded by brain regions other than the nucleus accumbens

4.7 Role of the thrombospondin receptor $\alpha 2 \delta 1$ in generation of cocaine induced silent synapses : A new direction to explore thrombospondin-mediated adult synaptogenesis.

4.8 Conclusions and outlook

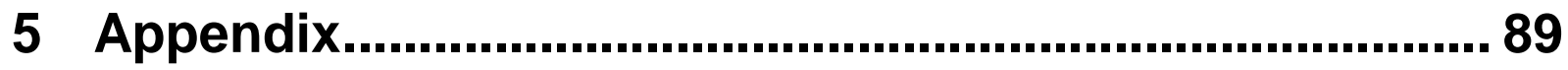

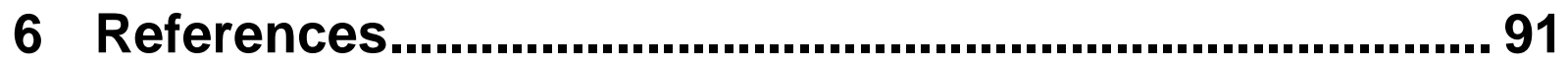

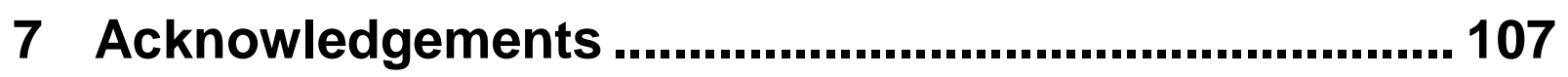

8 Curriculum Vitae .......................................................... 111 


\section{Abstract}

Drugs of abuse such as cocaine are known to hijack synaptic plasticity mechanisms of brain circuits underlying motivated behavior. A common mechanism of drugs of abuse is to elevate extracellular dopamine levels in the nucleus accumbens (NAc), a brain region of the ventral striatum that is a central part of the brain's reward circuit, the mesocorticolimbic dopamine system. NAc synapses are considered as major substrates for neural adaptions underlying drug reward. It has been shown that in vivo cocaine exposure rejuvenates the NAc neural network by generating new 'silent synapses'. These synapses lack stably integrated AMPA receptors and are hence 'silent' or non-transmitting at resting potentials. However, they bear the potential to be integrated into the neural circuit by switching to transmitting synapses, through means of AMPA receptor incorporation. So far, the mechanism with which cocaine exposure induces silent synapses in the NAc remains unknown. My results show that the function of $\alpha 2 \delta 1$, the receptor for synaptogenic astrocyte-secreted proteins called thrombospondins, in the NAc, is essential for the generation of silent synapses in adult mice following in vivo cocaine exposure. This brings to light the potential role of a pathway involving astrocyte-mediated synaptogenesis after cocaine exposure.

In this study, I used cocaine-conditioned place preference (cocaine-CPP), a behavioral assay to test the positive association of drug experience in mice. The association of cocaineinduced pleasure with environmental cues, during passive administration of cocaine, was measured as a preference of the mice to stay longer in the cocaine-paired compartment of the CPP apparatus. The long-term retention of this association, after a period of prolonged withdrawal from the drug, was used as a measure of long-term drug-associated memory. This memory was robust and long-lasting as mice retained the preference at least till 45 days of withdrawal from cocaine-CPP. To assess the changes in synaptic plasticity at cellular level, ex vivo electrophysiological measurements from NAc slices of mice were performed at defined time points of withdrawal from cocaine-CPP. My findings indicate that silent synapses are induced in the NAc by cocaine-CPP and mature during long-term withdrawal from cocaine-CPP by gradual incorporation of GluA2-lacking calcium-permeable AMPA receptors (CP-AMPARs). The association learnt by the mice during the cocaine-CPP paradigm, was required for the accumulation of CP-AMPARs in the NAc synapses. On a molecular level, the function of the DLG-MAGUK proteins - PSD-95 and SAP102 was required for the same. PSD-95 KO mice have a high proportion of immature silent synapses in the NAc, which do 
NOT accumulate CP-AMPARs during withdrawal from cocaine-CPP. These mice also lack long-term retention of the association learnt from the CPP paradigm. However, the loss of PSD-95 and also SAP102, only from NAc synapses, despite blocking CP-AMPAR accumulation, does NOT impair the long-term retention of drug-associated memory. Therefore contrary to the current understanding, the expression and accumulation of $\mathrm{CP}$ AMAPARs in the NAc synapses was NOT essential for long-term retention of this drugassociated memory. Furthermore, after prolonged withdrawal from cocaine selfadministration, the increase in cue-induced cocaine seeking, known as incubation of craving was NOT affected by NAc-restricted loss of PSD-95. Thus accumulation of CP-AMPARs in NAc synapses is a signature of associative memories of drug experience however, it is NOT essential for long-term retention of memory. These results draw attention to the fact that cellular correlates of memory traces pertaining to drug-associated cues may be stored in brain regions of the reward circuit other than the NAc. 


\section{List of Figures}

Figure 1.1 Simplified representation of the mesocorticolimbic dopaminergic system with specific pathways highlighted. 15

Figure 3.1 Wild type mice learn and remember the association with the cocaine-paired chamber in the CPP paradigm, up till 45 days after last cocaine injection.

Figure 3.2 Electrophysiological assays performed in the cells of the nucleus accumbens shell (NAcSh) show that withdrawal from cocaine-CPP is accompanied by a decrease in number of silent synapses and accumulation of calcium-permeable rectifying AMPARs.

Figure 3.3 Calcium-permeable AMPARs accumulate during withdrawal from cocaine only when the association is learnt in the cocaine-CPP assay.

Figure 3.4 Positive allosteric modulation of mGluR1 through systemic SYN119 injection resilences the NAc synapses after long-term withdrawal from cocaine by removal of CP. AMPARs. .50

Figure 3.5 PSD-95 KO mice learn well the association of the cocaine-paired chamber in the CPP paradigm but not retain this memory after 20 days. They have a high baseline number of silent synapses in the NAcSh which increases upon cocaine-CPP but is NOT converted to rectifying CP-AMPAR-containing synapses upon long-term withdrawal from cocaine. 52

Figure 3.6 SAP102 KO mice learn and remember the association of the cocaine-paired chamber in the CPP paradigm. Silent synapses in these mice increase in number after cocaine-CPP but are NOT converted to rectifying CP-AMPAR-containing synapses upon long-term withdrawal from cocaine.

Figure 3.7 Summarized results of CPP behavior assay (A) and electrophysiological assays (B) performed on NAcSh cells of WT, PSD-95KO and SAP102 KO mice. 56

Figure 3.8 NAcSh-restricted PSD-95 knock down does not impair long-term retention of CPP memory in wild type mice and SAP102KO mice. .59 
Figure 3.9 NAc restricted PSD-95 knock down has a cellular phenotype in wild type mice and SAP102KO mice. 62

Figure 3.10 NAcSh-restricted knockdown of PSD-95 does not impair self-administration of

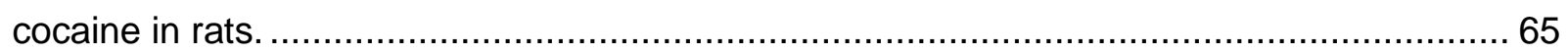

Figure 3.11 NAc-restricted knockdown of PSD-95 does not impair incubation of cocaine craving. 68

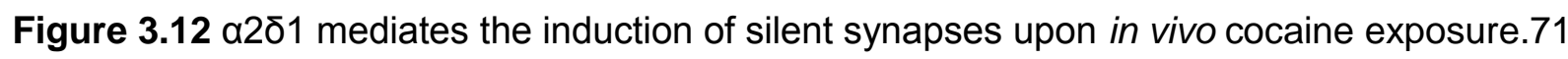

Figure 4.1 The dissociation between cocaine induced synaptic plasticity in the NAc (green box) and drug-associated long-term memory retention (red box) ..................................... 86 


\title{
List of abbreviations
}

\author{
AMPAR \\ a-amino-3-hydroxy-5methyl-4-isoxazolepropionic acid receptor \\ BLA \\ Basolateral amygdala \\ CP-AMPARs \\ Calcium permeable - AMPA receptors \\ CPP \\ Conditioned place preference \\ CS \\ Conditioned stimulus \\ DAT \\ Dopamine transporter \\ DLG-MAGUKs \\ DMEM \\ Disc large (DLG) protein family of membrane associated guanylate \\ kinases (MAGUKs) \\ EPSC \\ Dubelco's Modified Eagle Medium \\ FBS \\ Evoked excitatory postsynaptic current \\ GABA \\ Fetal bovine serum \\ GPCR \\ $\mathrm{Y}$-aminobutyric acid \\ i.p \\ G protein-coupled receptors \\ i.v \\ Intra-peritoneal \\ Intra-venous \\ KAR \\ Kainic acid receptors \\ KO \\ Knock-out \\ LB \\ Luria-Bertani or lysogeny broth \\ LTD \\ Long term depression \\ LTP \\ Long term potentiation \\ mGluR \\ Metabotropic glutamate receptor \\ MSNs \\ Medium spiny neurons \\ NAC \\ Nucleus accumbens \\ NAcSh \\ Nucleus accumbens shell \\ NMDAR \\ $\mathrm{N}$-methyl-D-aspartic acid receptor \\ PBS \\ Phosphate buffer saline \\ PSD \\ Postsynaptic density \\ PSD-95 \\ Postsynaptic density protein 95 \\ RT \\ Room temperature \\ SA \\ Self-administration \\ SH3 domain \\ SRC homology 3 domain
}




$\begin{array}{ll}\text { TARP } & \text { Transmembrane AMPAR regulatory domain } \\ \text { TSP } & \text { Thrombospondin } \\ \text { US } & \text { Unconditioned stimulus } \\ \text { vHipp } & \text { Ventral Hippocampus } \\ \text { VTA } & \text { Ventral tegmental area } \\ \text { WD } & \text { Withdrawal day } \\ \text { WT } & \text { Wild-type }\end{array}$




\section{Introduction}

The mammalian brain is comprised of an enormous network of billions of neurons, which communicate with each other, via specialized contacts called synapses. Electrochemical signals at these synapses, govern bodily functions and behavior. Modulation of synapse number and strength, resulting in alterations of neural connectivity, is considered to be the basis for learning and memory.

\subsection{Synaptic transmission}

A neuron typically possesses a soma (cell body), several dendrites and an axon. Neurons communicate with one another, through functional contacts termed synapses. Synapses can be broadly divided into two types - electrical and chemical.

Electrical synapses are gap junctions which allow fast transfer of information through a continuum of cytoplasm between two neurons (Bennett and Zukin, 2004). Rapid bidirectional electrical coupling through such synapses is important for synchronizing neuronal populations, such as those in the brainstem for generating breathing patterns (Rekling et al., 2000). However passive ionic flow through gap junctional channels restricts the possibility of regulating the strength of these synapses.

The vast majority of synapses in the vertebrate brain are chemical synapses. Chemical synapses are asymmetrical cellular junctions that have no cytoplasmic continuity. Instead, the presynaptic neuron and its target, which could be a postsynaptic neuron, muscle or gland, are separated by a 20-40 nm gap called the synaptic cleft (Palay, 1956; Burns and Augustine, 1995). Electrical signals, in the form of action potentials propagating through the axons, invade the presynaptic bouton and are converted to chemical signals. Biochemical messenger molecules called neurotransmitters, serve as the chemical signals, as they are released from the presynaptic bouton into the synaptic cleft and bind to and activate, neurotransmitter-gated ion channels on the post-synaptic membrane. Opening of these postsynaptic channels, allows ionic influx, which alters the membrane potential of the postsynaptic neuron. Thus the signal is converted back to electrical impulses within the postsynaptic dendrite. The summation of such membrane potentials occurs at the cell soma, and if it exceeds the action potential threshold, a new action potential is generated in this neuron which propagates along its axon. Chemical synapses have a very high capacity for 
regulation and modulation as synaptic transmission across chemical synapses is highly complex and extremely tightly regulated (Wojcik and Brose, 2007; Scannevin and Huganir, 2000).

\subsubsection{Presynaptic neurotransmitter release}

At the presynaptic bouton, neurotransmitters are stored in small synaptic vesicles, a subset of which are recruited to "active zones" that are specialized release-ready sites at the presynaptic membrane (Gray, 1963). Each active zone comprises a dense network of protein complexes that mediate synaptic vesicle mobilization as well as refilling and facilitate fast synchronous release of neurotransmitter (Sudhof, 2004; Wojcik and Brose, 2007).

Depolarization of the presynaptic neuronal membrane, results in the sequential opening of voltage gated sodium channels and spread of an action potential along the axon. When the action potential reaches the presynaptic bouton, it causes the opening of voltage-gated calcium channels. The influx of $\mathrm{Ca}^{2+}$ ions into the cell raises the intracellular concentration of $\mathrm{Ca}^{2+}$, which in turn leads to SNARE-mediated fusion of the synaptic vesicle membrane and the presynaptic plasma membrane. Hence an action potential triggers neurotransmitter release into the synaptic cleft by exocytosis of synaptic vesicles (Evans and Zamponi, 2006; Reid et al., 2003; Catterall and Few, 2008).

\subsubsection{Types of neurotransmitters}

Neurotransmitters can be single amino acids - glutamate, glycine and GABA ( $\gamma$ aminobutyric acid); biogenic amines - dopamine, serotonin, epinephrine or peptides - for e.g., endorphins. However, depending on the type of receptor neurotransmitters interact with on the postsynaptic membrane, they can be functionally categorized as excitatory, inhibitory and modulatory -

\section{a) Excitatory neurotransmitters - glutamate}

Excitatory neurotransmitters bind to and open cation channels, primarily sodium and potassium channels, thereby causing depolarization of the postsynaptic membrane and increasing the likelihood that the postsynaptic neuron would fire an action potential. Glutamate is the principle excitatory neurotransmitter in the brain. 


\section{b) Inhibitory neurotransmitters - GABA, glycine}

Inhibitory neurotransmitters bind to and open chloride-specific channels, thereby hyperpolarizing the cell membrane and decreasing the likelihood that the postsynaptic neuron would fire an action potential.

c) Modulatory neurotransmitters - For e.g., dopamine, serotonin, epinephrine

Modulatory neurotransmitters act through secondary messengers and alter the postsynaptic neuron's metabolism and membrane permeability.

The neurotransmitter identity is a defining feature of a neuron, as one neuron typically contains one neurotransmitter. Each neuron however, is often innervated by multiple neurotransmitters, in accordance to the different types of synaptic connections and therefore neurons express the respective neurotransmitter receptors.

\subsection{Glutamatergic excitatory synapses}

The postsynaptic compartment of an excitatory synapse is formed on mushroom-like protrusions of dendrites, called dendritic spines. Macromolecular protein complexes within spine heads, form an electron-dense thickening, when viewed under the electron miscroscope, which is termed the post synaptic density (PSD) (Palay, 1956). The PSD is directly apposed to the presynaptic active zone and is comprised of neurotransmitter receptors, scaffold proteins, cell-adhesion molecules, cytoskeletal proteins and several other proteins for downstream signaling (Sheng, 2001a; Garner et al., 2002; Sheng and Hoogenraad, 2007).

\subsubsection{Glutamate receptors}

Neurotransmitter receptors can be broadly categorized into ionotropic receptors and metabotropic receptors. lonotropic receptors are ligand-gated ion channels that allow the opening of the channel directly upon ligand-binding (or in this case, neurotransmitter-binding) due to a conformational change in their protein structure. Owing to their rapid response to the ligand, they mediate very fast excitatory or inhibitory synaptic transmission. On the other hand, metabotropic receptors are G-protein coupled receptors (GPCRs) which are directly or 
indirectly linked to the effector ion channel through intracellular signaling of secondary messenger cascades that have multiple effects. Therefore they mediate slow or modulatory forms of synaptic transmission (Nicoll et al., 1990).

At the excitatory post-synaptic membrane, glutamate binds to its cognate ionotropic or metabotropic receptors -

\section{a) lonotropic glutamate receptors - AMPARs, NMDARs and KARs}

There are three families of ionotropic glutamate receptors - AMPARs ( $\alpha$-amino-3-hydroxy5methyl-4-isoxazolepropionic acid receptors), NMDARs ( $\mathrm{N}$-methyl-D-aspartic acid receptors) and KARs (Kainic acid receptors); each named after its respective synthetic agonist. AMPARs and KARs mediate the fast or early component of excitatory postsynaptic currents (EPSCs), whereas NMDARs which have higher conductance but slower kinetics, mediate the slow or late component (Stern et al., 1992). Unlike AMPARs and KARs, which are directly activated by glutamate binding, NMDARs are coincident detectors. At resting membrane potentials $(-60$ to $-70 \mathrm{mV})$, the ion pore of NMDARs is blocked by extracellular $\mathrm{Mg}^{2+}$. This block is voltage sensitive, and can be relieved upon membrane depolarization. Therefore membrane depolarization along with binding of glutamate and glycine which is a necessary co-agonist, is needed for NMDARs to open and allow influx of $\mathrm{Na}^{+}$and $\mathrm{Ca}^{2+}$ and efflux of $\mathrm{K}^{+}$ ions. Therefore, gating of NMDARs require the coincident detection of pre- and postsynaptic activity (Stern et al., 1992; Kleckner and Dingledine, 1988; Laube et al., 1998). $\mathrm{Ca}^{2+}$ entry triggers several downstream signaling events that are important for synapse modulation (Berridge, 1998).

Ionotropic glutamate receptors are tetramers and their properties are defined by their subunit composition and their modifications (Dingledine et al., 1999; Dingledine and Conn, 2000).

AMPARs are hetero-oligotetramers of four subunits (Hollmann and Heinemann, 1994) GluA1 to GluA4 (also known as GluR1 to GluR4 or GluRA-D, according to previous nomenclature (Collingridge et al., 2009)). The exact subunit composition of AMPARs differs between brain regions, however in hippocampus and cortex, majority of the AMPARs are GluA1/2 heterodimers and remainder are mostly GluA2/3 heterodimers (Wenthold et al., 1996; Mansour et al., 2001; Lu et al., 2009). GluA4-containing AMPARs are present in immature neurons of the hippocampus, very early in development and are replaced by 
GluA2-containing AMPARs (Zhu et al., 2000). GluA2-lacking AMPARs, i.e. GluA1/3 heterodimers and to a lesser extent, GluA1 homomers, can also be assembled (Wenthold et al., 1996). The properties of GluA2-lacking AMPARs are notably different from GluA2containing AMPARs, as they are calcium permeable or bear significant $\mathrm{Ca}^{2+}$ conductance (they are also referred to as CP-AMPARs - calcium permeable AMPARs). The GluA2 subunit undergoes vigorous RNA editing, a post-translational modification that substitutes a glutamine residue with an arginine, thereby rendering GluA2-containing AMPARs, which are the majority, impermeable to $\mathrm{Ca}^{2+}$ (Seeburg et al., 1998).

NMDARs are heterotetramers composed of a combination of subunits namely GluN1, GluN2A, GluN2B, GluN2C, GluN2D (also called NR1, NR2A, NR2B, NR2C, NR2D according to previous nomenclature (Collingridge et al., 2009)) and the more distally related members GluN3A and GluN3B. NMDARs typically contain two obligatory GluN1 subunits with two GluN2 subunits or one GluN2 and one GluN3 subunit (Traynelis et al., 2010). In the forebrain, GluN2A and GluN2B are the most abundant, whereas GluN2C and GluN2D are abundant in the cerebellum and superior colliculus respectively (Goebel and Poosch, 1999). NMDARs at birth are comprised of predominantly GluN1/GluN2B subunits, however during early postnatal development there is a switch to preferentially GluN2A-containing NMDARs, which is regulated in an experience and activity-dependent manner (Sheng et al., 1994; Quinlan et al., 1999; Liu et al., 2004; Gray et al., 2011). GluN2A and GluN2B assemble into canonical NMDA heterodimers or heterotrimers with slower decay time kinetics than AMPARs, high $\mathrm{Ca}^{2+}$ flux and strong voltage-dependent $\mathrm{Mg}^{2+}$ block. However the less extensively studied, GluN3A assembles into non-.canonical NMDARs having lower conductance, lower $\mathrm{Ca}^{2+}$ permeability and reduced $\mathrm{Mg}^{2+}$ sensitivity (Tong et al., 2008; Paoletti, 2011).

AMPARs and NMDARs are enriched at excitatory PSDs, while KARs can be localized to pre-and postsynaptic sites and can modulate synaptic activity. AMPARs and KARs share several agonists and antagonists and are therefore collectively referred to as non-NMDA receptors. KAR mediated synaptic transmission was primarily found in hippocampal mossy fibre synapses, where it has been extensively studied. Unifying features of all KAR-mediated synaptic current is that it is much smaller than AMPA currents and also much slower, owing to the slower deactivation kinetics of KARs (Lerma, 2003; Traynelis et al., 2010). 


\section{b) Metabotropic glutamate receptors - mGluRs}

There are 8 mGluRs with a wide distribution all over the brain, some of which are both preand postsynaptically localized. mGluRs activate a myriad of different signaling pathways that can in turn, modulate neuronal excitability and neurotransmitter release. Functionally, they are categorized into three groups- group I mGluRs (including mGluR1 and mGluR5, and their splice variants) are primarily expressed postsynaptically in perisynaptic zones surrounding ionotropic receptors (Lujan et al., 1996); group II mGluRs (including mGluR2 and mGluR3) are located at pre-and postsynaptic sites; and group III mGluRs (including mGluR4, mGluR6, mGluR7 and mGluR8) are primarily presynaptic. In broad terms, activation of group I mGluRs is positively coupled to phosphoinositide hydrolysis through Gq protein and leads to enhanced neuronal excitation. On the contrary, activation of group II and group III mGluRs is negatively coupled to adenylate cyclase through Gi protein and negatively modulates glutamate release (Pomierny-Chamioło et al., 2014; Cartmell and Schoepp, 2000).

\subsubsection{Structural and functional PSD scaffolds}

The PSD contains several PDZ-domain-containing scaffold proteins. PDZ domains are modular protein-protein interaction domains, specialized for binding to specific sequences in the extreme C-terminal motifs of other proteins, thereby mediating protein targeting and multiprotein complex assembly. The PDZ-domain containing proteins at the PSD are involved in clustering of glutamate receptors at the cell surface and their trafficking, organization of protein complexes for downstream signaling as well as cytoskeletal dynamics (Feng and Zhang, 2009; Kim and Sheng, 2004). The DLG-MAGUK (disc large membrane associated guanylate kinases) family of proteins plays a prominent role in glutamate receptor trafficking (Elias et al., 2006). The proteins of this family share similar domain structures consisting of three PDZ domains, one SH3 (src homology 3 domain) and an enzymatically inactive GK (guanylate kinase) domain. There are four members of this family - PSD-95 (postsynaptic density protein of 95kD MW), PSD-93, SAP-97 (synapse associated protein 97) and SAP102.

PSD-95 is present in particularly high abundance in the PSD (Cho et al., 1992; Kistner et al., 1993; Peng et al., 2004). It plays a dominant role in governing synapse strength, due its influence on AMPAR trafficking and retention at the synapse (Ehrlich and Malinow, 2004; Schlüter et al., 2006). At first it was discovered that PSD-95 directly binds to a C-terminal motif on NMDAR subunits, thereby stabilizing NMDARs at synapses (Kornau et al., 1995; 
Roche et al., 2001; Sheng, 2001b). However PSD-95 also binds, though indirectly, to AMPARs through Stargazin (an auxillary subunit or TARP - transmembrane AMPAR regulatory protein) thereby mediating AMPAR delivery to synapses (Chen et al., 2000; Schnell et al., 2002). PSD-95 was shown to be responsible for maturation of glutamatergic synapses by clustering of AMPARs, but not NMDARs, at synapses (El-Husseini et al., 2000). Overexpression of PSD-95 or replacing endogenous PSD95 with its major isoform, PSD-95a, increases AMPAR-mediated synaptic transmission (Schnell et al., 2002; Ehrlich and Malinow, 2004; Schlüter et al., 2006). This was concomitant with results from RNAi-mediated knockdown of PSD-95, which resulted in a decrease of synaptic strength due to reduction of AMPAR-mediated EPSCs to about 50\%, while NMDARs EPSCs were largely unaffected (Elias et al., 2006; Schlüter et al., 2006).

Studies using knock-out (KO) mice of different DLG-MAGUKs, have revealed that the proteins of this family have compensatory roles. This explains why PSD-95KO, PSD-93 KO and SAP102 KO mice have no impairments in basal excitatory transmission (Migaud et al., 1998; McGee et al., 2001; Cuthbert et al., 2007; Elias et al., 2006). PSD-95/PSD-93 double knock-out (DKO) mice though, have impaired AMPAR-mediated basal transmission (Elias et al., 2006). SAP102 is usually the predominant MAGUK in immature synapses present during early development, however it is significantly upregulated in mature synapses of PSD-95 KO mice and PSD95/PSD-93 DKO mice. Thus, SAP102 functionally compensates for the lack of PSD-95 (Elias et al., 2006; Cuthbert et al., 2007; Bonnet et al., 2013).

\subsubsection{Synaptic plasticity - cellular basis of learning and memory}

The ability of synapses to change their strength in an activity-dependent manner is termed as synaptic plasticity. Owing to this ability, synapses can be strengthened or weakened which forms the basis of learning and memory. Two forms of plasticity that have been extensively studied are - LTP (long-term potentiation) and LTD (long-term depression). LTP is an activitydependent, long-lasting increase in synaptic transmission. It was first discovered in the hippocampus using brief high-frequency bursts of stimulation (Bliss and Lømo, 1973) and subsequently shown to be induced in vivo, by learning (Whitlock et al., 2006). LTD on the other hand, is an activity-dependent long-lasting decrease in synaptic transmission, hence functionally the opposite of LTP. It was also shown at first in the hippocampus, using a lowfrequency stimulation (Dudek and Bear, 1992). By now, it is widely accepted that LTP and 
LTD are basic properties of most excitatory synapses and that these synaptic plasticity mechanisms are involved in several brain functions.

\subsubsection{Common forms of LTP and LTD}

NMDAR-dependent LTP - The form of LTP which was first described in the hippocampus, was found to be NMDAR-dependent (Collingridge et al., 1983) and remains to be the most extensively studied form of LTP. It is a type of Hebbian plasticity, which is induced upon coincidence detection of pre- and postsynaptic activity by NMDARs. NMDARs in postsynaptic dendritic spines are activated and flux $\mathrm{Ca}^{2+}$, only when presynaptically released glutamate binds the receptor and the postsynaptic membrane is sufficiently depolarized. The postsynaptic dendritic membrane may be depolarized due to backpropagating action potentials (Magee and Johnston, 1997) or dendritic spikes, which are locally generated and spatially restricted action potentials (Golding et al., 2002). Postsynaptic NMDA activation results in a rapid increase of $\mathrm{Ca}^{2+}$ concentration in the dendritic spine, which triggers downstream signaling cascades involving several protein kinases. CaMKII (calciumcalmodulin protein kinase II) is a critical target of $\mathrm{Ca}^{2+}$ because its autophosphorylation results in its CaM-independent activity that is both necessary and sufficient for LTP (Pettit et al., 1994; Giese et al., 1998). CamKII has several important downstream targets, including AMPAR subunits. It has been shown by several studies that phosphorylation of certain AMPAR subunits and their TARPs, alters AMPAR trafficking (Boehm and Malinow, 2005; Straub and Tomita, 2012) and conductance (Kristensen et al., 2011), thereby contributing to LTP. Ultimately, through mechanisms that are still under debate (Shi et al., 2001; Granger et al., 2013; Nicoll and Roche, 2013), AMPARs are inserted into the synapse. Thus an increase in the number of synaptic AMPARs, results in strengthening or potentiation of a glutamatergic synapse. From a structural perspective, LTP results in an increase in spine volume and enlargement of the PSD (Matsuzaki et al., 2004; Yuste and Bonhoeffer, 2001).

NMDAR-dependent LTD - Weak activation of synaptic NMDARs due to low stimulation frequencies or modest membrane depolarization, leads to NMDAR-dependent LTD (Dudek and Bear, 1992; Mulkey and Malenka, 1992). The rise of postsynaptic $\mathrm{Ca}^{2+}$ concentration needed for LTD, is lesser than that needed for LTP (Mulkey and Malenka, 1992), and leads to signaling cascades that are distinct from LTP. The activation of serine/threonine phosphatases is critical (Mulkey et al., 1993, 1994), as it leads to de-phosphorylation of key substrates, including AMPARs (Lee et al., 1998, 2000). This ultimately results in removal of 
AMPARs from the postsynaptic membrane, via dynamin- and clathrin-mediated endocytosis (Beattie et al., 2000; Carroll et al., 1999, 2001; Wang and Linden, 2000), though mechanistic details are still not very clear (Granger and Nicoll, 2014).

mGluR-dependent LTD - Several forms of mGluR-LTD have been observed, in various brain regions following activation of different mGluRs. However in these kinds of synaptic depression, the mechanism as well as the locus of induction and expression of LTD, can differ depending on the brain region. mGluR-LTD can be induced and expressed at the postsynapse (for e.g. by mGluR1, mGluR5 and mGluR2); or at the presynapse (for e.g. by mGluR2 and mGluR7); and can also be postsynaptically induced and presynaptically expressed (for e.g. by mGluR1/5)(Bellone et al., 2008). Groupl mGluRs (mGluR1 and mGluR5) which are perisynaptically located (not within the PSD), are activated by glutamate spillover outside the synaptic cleft and mediate LTD in several brain regions such as hippocampus, cerebellum, ventral tegmental area (VTA). mGluR-LTD was first described at cerebellar synapses wherein climbing fibers and parallel fibers that converge onto purkinje cells, need to be activated together, to depolarize purkinje cells during mGluR1 activation (Ito, 1989). This results in internalization of synaptic AMPARs and hence reduced synaptic efficacy. mGluR1-LTD can also be achieved without reduction in synaptic AMPAR number. At the VTA and cerebellar stellate cells, mGluR1-LTD results in replacement of CP-AMPARs (calcium-permeable AMPARs that lack GluA2 subunit) with Cl-AMPARs (calciumimpermeable AMPARs that contain GluA2 subunit). Since Cl-AMPARs have a lower conductance than CP-AMPARs, this exchange results in weakening of synaptic transmission (Bellone and Lüscher, 2005; Mameli et al., 2007; Kelly et al., 2009). At synapses of certain brain regions, groupl mGluR activation can trigger the synthesis of endocannabinoids (eCB) which act as retrograde messengers and diffuse across the synaptic cleft to bind presynaptic CB1 receptors and ultimately lower glutamate release probability. For e.g., this form of presynaptically expressed eCB-LTD has been found in the striatal medium spiny neurons of the nucleus accumbens, where it is induced postsynaptically by mGluR5 activation (Robbe et al., 2002).

\subsubsection{Role of PSD-95 in synaptic plasticity}

It was observed that trafficking of AMPARs to the postsynaptic membrane, by itself is not sufficient for synaptic potentiation, as the AMPARs are inserted into extrasynaptic sites (Oh et 
al., 2006; Schnell et al., 2002). For reliable synaptic transmission, AMPARs need to be clustered at the PSD, directly opposite to presynaptic release sites (Lisman and Raghavachari, 2006). There is lot of evidence suggesting that AMPARs laterally diffuse from a pool of pre-existing or newly-inserted AMPARs in extra/perisynaptic sites, into synaptic sites, where they are anchored into affinity 'slots' available in the PSD (Tardin et al., 2003; Opazo and Choquet, 2011; Opazo et al., 2012). The PSD-95-Stargazin interaction plays a dominant role in AMPAR recruitment to synapses (Tomita et al., 2005; Schnell et al., 2002; Chen et al., 2000). However, PSD-95 functions not just as a slot, but being a scaffolding protein, it orchestrates several downstream signaling events by mediating protein-protein interactions ( $\mathrm{Xu}$ et al., 2008). Indeed overexpression of PSD-95 is sufficient to potentiate synaptic transmission (Ehrlich and Malinow, 2004; Stein et al., 2003) and loss of PSD-95 from spines increases AMPAR mobility and leads to synaptic depression (Arendt et al., 2010). Synaptic plasticity is also impaired in PSD-95 KO mice. These mice have enhanced LTP in the hippocampal slices and perform poorly in watermaze behavioral tasks, showing an underlying defect in learning and memory (Migaud et al., 1998). Also, acute knock-down of PSD-95 greatly reduces the magnitude of LTD (Xu et al., 2008) and in the PSD-95 KO mice LTD is absent (Migaud et al., 1998).

\subsubsection{Silent synapses and plasticity}

While NMDARs are anchored in a relatively stable manner at the synapse, AMPARs are more dynamically regulated and move in and out of the synapse in an activity -dependent manner (Shepherd and Huganir, 2007). Although typical glutamatergic synapses contain both AMPARs and NMDARs, there are some synapses that contain only NMDARs and lack stable AMPARs. These are called 'silent synapses' because they are functionally silent or nonconducting near resting membrane potentials, owing to the voltage-dependent $\mathrm{Mg}^{2+}$ block on NMDARs (Petralia et al., 1999; Kerchner and Nicoll, 2008; Hanse et al., 2013). Also, these synapses may contain AMPARs that are highly labile and therefore, not functionally integrated into the synapse (Groc et al., 2006). Such synapses were first proposed as 'ineffective synapses' between primary afferent fibers and spinal cord dorsal horn (Merrill and Wall, 1972). Subsequently, there were several reports of the presence of silent synapses in hippocampus and other brain regions using electrophysiological methods (Kullmann, 1994; Isaac et al., 1995; Liao et al., 1995), as well as biochemical and morphological techniques (Richmond et al., 1996; Nusser et al., 1998; Petralia et al., 1999). Moreover, glutamate 
uncaging experiments have proven that such synapses are postsynaptically silent, without modifications in presynaptic glutamate release (Busetto et al., 2008). Silent synapses though low in number in the mature brain, are very abundant at the earliest stages of development and are sometimes referred to as nascent synapses. These can subsequently undergo maturation during development, to form functional synapses. Correlated pre- and postsynaptic activity drives AMPARs into silent synapses, thereby unsilencing them through LTP-like mechanisms (Durand et al., 1996). Indeed unsilencing of synapses and LTP seems to be an identical phenomenon as conventional pairing protocols used to induce LTP, can also unsilence silent synapses, through mechanisms requiring NMDA activation and CaMKII activity (Liao et al., 1995; Wu et al., 1996; Isaac et al., 1997). This postsynaptic maturation by means of unsilencing of silent synapses, plays a key role in activity- and sensory-dependent refinement of neural circuits (Isaac et al., 1997; Huang et al., 2015a). Alternatively, silent synapses can also be generated by removal or de-stabalization of synaptic AMPARs (Xiao et al., 2004). These silent synapses bear a huge potential for housing incoming AMPARs in a subsequent step of plasticity. This step of plasticity can be more quick and durable, and is often referred to as metaplasticity (Abraham and Bear, 1996).

Just as PSD-95 being a critical player for postsynaptic maturation, is important for LTP, it also seems to be important for unsilencing of silent synapses. PSD-95 KO mice have a high proportion of silent synapses in the adult brain (Béïque et al., 2006; Huang et al., 2015a). Since PSD-95 is crucial for AMPAR retention at the PSD, lack of PSD-95 probably renders AMPARs labile and the synapse immature. Moreover these studies suggest a synapsespecific role of PSD-95 in experience-driven maturation of silent synapses. A significant population of cells ( 50\%) was shown to stabilize AMPARs independent of PSD-95, while the others were dependent on PSD-95 to mature in an activity-dependent manner.

\subsection{Role of astrocytes in synaptogenesis}

Apart from neurons, there are other major cell types of the brain are subsumed under the term glia. Glial cells are divided into three types - astrocytes, oligodendrocytes and microglia. They are known to provide mechanical and functional support to neurons. Oligodendrocytes perform the function of myelinating axons, thereby greatly influencing the speed of neuronal action potential propagation (Edgar and Nave, 2009). Microglia being neuroprotective and immunocompetent, have a specialized role in host defense (Streit, 2002). The roles of 
astrocytes however, are quite diverse and have radically evolved over the past years. Apart from being support cells, they ensure optimal functioning of neurons. Astrocytes modulate synaptic efficacy through the release and uptake of neuroactive substances. They are excitable and release 'gliotransmitters' including glutamate, which act on mGluRs to modulate synaptic transmission. They are also known to regulate blood flow and be involved in synapse formation (Volterra and Meldolesi, 2005; Barres, 2008).

Of particular interest to this study, is the role of astrocytes in modulating synapse numbers (Ullian et al., 2001, 2004). It was postulated that synaptogenesis is stimulated by astrocytesecreted pro-synaptogenic signals (Meyer-Franke et al., 1995; Pfrieger and Barres, 1997). Subsequently it was discovered that astrocyte secreted glycoproteins called thrombospondins (TSPs) induce synaptogenic signals (Eroglu, 2009). TSP1 and TSP2, which are highly expressed by immature astrocytes during the synaptogenic period of the mouse brain (postnatal day 5-10), are necessary and sufficient for stimulating excitatory synaptogenesis (Christopherson et al., 2005; Xu et al., 2010). These TSP-induced synapses were seen to be ultrastructurally normal and postsynaptically silent (lacking stable synaptic AMPARs), as is the case during early development (Christopherson et al., 2005). Moreover, $\alpha 2 \delta-1$, which is a voltage-gated calcium channel subunit, was identified as the neuronal thrombospondin receptor, which is essential for mediating TSP-induced synaptogenesis (Eroglu et al., 2009). Binding of TSPs, through their common epidermal growth factor (EGF)-like repeats, to the extracellular Von Willebrand Factor A (VWF-A)-like domain of $\alpha 2 \delta-1$, is thought to induce an intracellular signaling cascade (which is yet unknown) and ultimately leads to synapse formation (Eroglu et al., 2009; Procko and Shaham, 2009; Risher and Eroglu, 2012). Furthermore, $\alpha 2 \delta-1$ is also a high affinity receptor for gabapentin, a common anti-epileptic and analgesic drug (Gee et al., 1996). Thus gabapentin can disrupt TSP- a2ס-1 binding and block new synapse formation both in vitro and in vivo (Eroglu et al., 2009).

\subsection{Drug addiction and synaptic plasticity}

Drug addiction is a devastating disease, characterized by compulsive drug use, in spite of serious negative consequences. Addicted individuals value the drug over all other rewards in life and therefore maintain a narrowed focus on obtaining and using drugs. This is a chronically relapsing disorder, as individuals once addicted, remain highly prone to relapse, long after cessation of drug use and despite repetitive episodes of treatment. Therefore more effective treatment interventions are much needed for this persistent illness, as no treatment yet, is considered to be completely curative (McLellan A et al., 2000). 
The addiction cycle is thought to comprise of three stages - (1) binge/intoxication i.e. the drug-taking phase often accompanied by escalated intake of the drug; (2) withdrawal/negative effect i.e. buildup of a negative emotional state due to lack of access to the drug; (3) preoccupation/anticipation i.e. the compulsive drug-seeking phase due to 'craving' for the drug (Koob and Volkow, 2009). The high propensity of relapse especially upon encounter with drug-related cues, following prolonged periods of withdrawal from the drug, suggests that a robust and pathological long-term form of associative memory underlies addiction.

Drugs of abuse achieve this power by hijacking the natural mechanisms of reward-related learning and memory (Hyman et al., 2006a). Rewards are interpreted by the brain, as objects or actions possessing positive values. Behaviors which are rewarding, tend to be repeated over time, which is known as reinforcement learning (Montague et al., 2004). Natural rewards such as food and sex are beneficial for the survival of the organism and continuation of its species. Therefore organisms learn to obtain food and find mating opportunities. The responses to natural rewards and drugs of abuse have some similarities such as, the induced pleasure and feelings of desire. However drugs of abuse, being both rewarding and reinforcing, are far more harmful, as they provide no health or evolutionary benefit, but are still overvalued compared to other rewards (Kelley and Berridge, 2002).

\subsubsection{Behavioral tests for drug reinforcement in rodents}

Though it is very difficult to reproduce the phenomenon of addiction in laboratory settings, there are methods that measure the reinforcing properties of drugs and those that evaluate addictive behavior in laboratory animals (Sanchis-Segura and Spanagel, 2006).

Two commonly used methods for testing drug-induced reinforcement are conditioned place preference (CPP) and operant self-administration (SA). CPP as well as other tests based on conditioned preference, entail passive administration of drugs by the experimenter. Conditioned preference tests can be considered Pavlovian in nature. The drug's effects (the unconditioned stimulus, US) are repeatedly paired with a previously neutral stimulus, which eventually becomes a conditioned stimulus (CS) and can elicit approach behavior of the animal. In place conditioning methods, environmental stimuli act as the CS. In CPP, the apparatus consists of two compartments wherein, only one becomes associated with the drug-experience, while the other is accessed only after vehicle or control administration. Subsequently, when the animal is allowed to freely move between the two compartments, the 
increase in the time spent in the drug-paired compartment is a measure of the conditioned place preference. A repetition of this test after a period of withdrawal from drug-administration can be done to test the long-term retention of the drug-associated memory (Roux et al., 2003; Cunningham et al., 2006; Sanchis-Segura and Spanagel, 2006).

The operant conditioning model of self - administration (SA) of the drug, is a Skinnerian concept wherein, behavior is controlled by its consequences. Positive reinforcement is achieved by the contingent delivery of the positive reinforcer, (in this case of SA, it is the drug) upon completion of a reinforcement schedule. Rodents are used most often for this model and for self-administration of psychostimulants like cocaine, the intravenous (i.v) route of drug administration is used, to induce rapid onset of drug action. Typically, rats are implanted with catheters in the jugular vein, for the i.v drug delivery and are trained to selfadminister the drug during short training sessions. The operant response, which can be the press of a lever or nose-poke into a hole, gets rewarded by instantaneous i.v infusion of the drug. Also, secondary reinforcers such as lights or sound tones can be used as cues for the operant response (Lynch et al., 2010; Sanchis-Segura and Spanagel, 2006). This procedure has been successfully used to model drug-craving behavior in rats during withdrawal from the drug (Grimm et al., 2001; Pickens et al., 2011).

\subsubsection{Hijacking of the reward circuits of the brain}

Drugs of abuse such as cocaine, hijack synaptic plasticity mechanisms of the brain circuits controlling motivated behavior. The mesocorticolimbic dopamine system (See Figure 1) is the reward circuitry in the brain, involved in positive reinforcement learning. It comprises of the ventral tegmental area (VTA) in the mid brain and the nucleus accumbens (NAc) in the ventral striatum, as well as associated cortical and limbic structures. Drugs of abuse cause long-term changes in behavior by altering synaptic function and plasticity in these brain areas (Hyman et al., 2006a; Kauer and Malenka, 2007). 


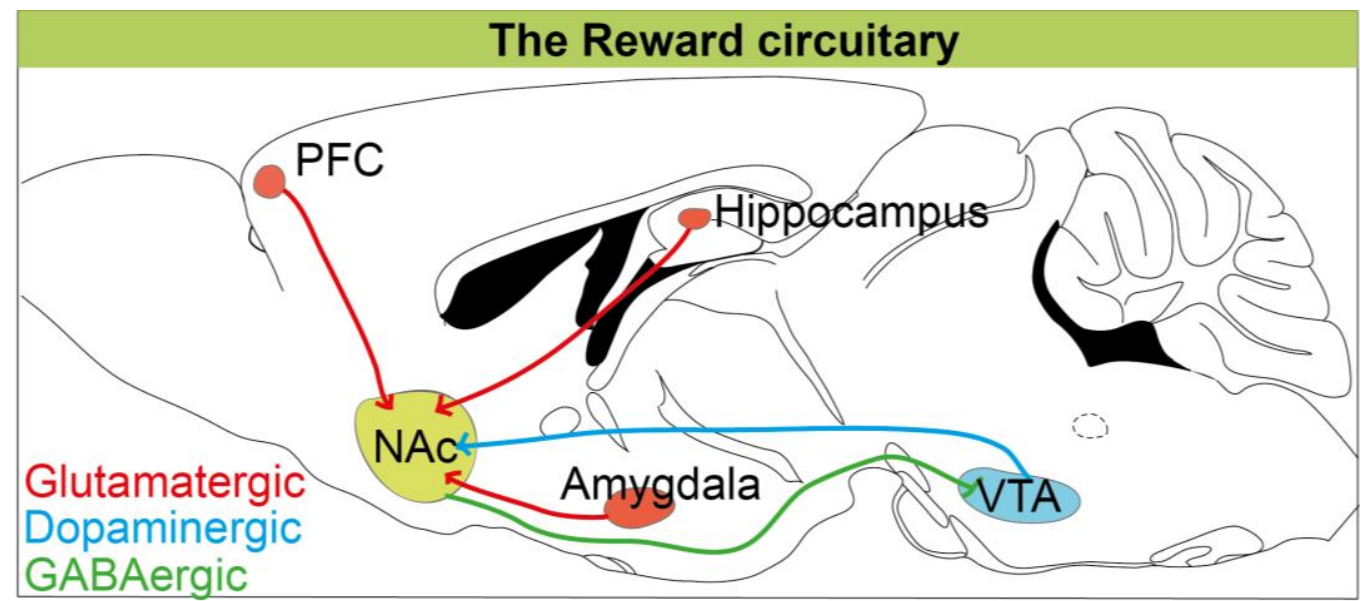

Figure 1.1 Simplified representation of the mesocorticolimbic dopaminergic system with specific pathways highlighted.

The nucleus accumbens (NAc) receives glutamatergic input from the medial prefrontal cortex (PFC), the ventral hippocampus and the amygdala. All four of these regions receive dopaminergic input from the ventral tegmental area (VTA) (although only the VTA-NAc pathway is shown here). The VTA receives GABAergic or inhibitory input from the NAc.

The dopaminergic projections from the VTA, onto the NAc is an essential pathway for reward-seeking behavior (Nicola, 2010; Niv et al., 2006; Saunders and Robinson, 2012). In fact the central mechanism of most drugs of abuse, is to elevate the extracellular dopamine levels in the NAc. Cocaine for example, blocks dopamine reuptake from the synaptic terminals by inhibition of the dopamine transporter (DAT) and thereby has rewarding and reinforcing properties (Chen et al., 2006; Ritz et al., 1987).

The NAc itself consists mainly of GABAergic medium spiny neurons (MSNs) which in turn project on to the VTA. Apart from dopaminergic input, the NAc also receives glutamatergic input from the medial prefrontal cortex (mPFC), the ventral hippocampus (vHipp) and the basolateral amygdala (BLA). Thus the NAc is a key component of the reward circuit as it is the point of convergence for these excitatory inputs, each of which signal distinct reward related information. In broad and simple terms, the mPFC provides action-outcome information, the vHipp contextual relevance and the BLA emotional valence (Robbins and Everitt, 1996a). These signals are integrated by the neurons of the NAc, which in turn guide appropriate behaviors in response to the signals, thereby serving as an interface between motivation and action (Mogenson et al., 1980; French and Totterdell, 2002, 2003; Papp et al., 2011). Drugs of abuse are known to cause maladaptive plasticity in glutamatergic signaling pathways of the reward circuit (Kalivas, 2004; Lüscher and Malenka, 2011; Pascoli et al., 2014). 
Synaptic plasticity in the VTA dopaminergic neurons, following initial drug exposure, is essential for mediating early behavioral responses and for long-term adaptations in the areas innervated, such as the NAc, pre-frontal cortex (PFC) and amygdala (Kauer, 2004). Rewardpredicting cues can induce phasic activation of VTA dopaminergic neurons, which in turn is regulated by glutamatergic input onto these neurons (Schultz, 2011; White, 1996). A single injection of cocaine causes potentiation of excitatory inputs onto VTA dopamine cells, as measured by an increase in AMPAR/NMDA EPSC ratio (Ungless et al., 2001). In fact most commonly used drugs of abuse, as well as natural rewards, share this mechanism of increased glutamatergic drive onto dopamine neurons (Saal et al., 2003; Chen et al., 2008). However a fundamental difference is that the potentiation of VTA glutamatergic signaling is persistent after cocaine self-administration, whereas it is only transient after selfadministration of food or sucrose (Chen et al., 2008).

Synaptic changes in the NAc are downstream to the VTA and take place at a slower time scale and steeper threshold, but are considered to be responsible for long-lasting behavioral alterations. Unlike the VTA, where one cocaine injection can elicit readily detectable plasticity, 5 injections of cocaine are required to see plasticity in the NAc (Kourrich et al., 2007). Repeated exposure to cocaine, was shown to induce an initial depression of glutamatergic transmission (Thomas et al., 2001), which upon prolonged withdrawal from the drug, is converted to a potentiation of AMPAR-mediated synaptic transmission (Kourrich et al., 2007).

\subsubsection{Cocaine-induced silent synapses in the adult brain}

It has been shown that silent synapses can be generated post-developmentally, in the adult mammalian brain, upon repeated in vivo exposure to cocaine. These 'AMPAR-silent' synapses were first seen to be generated in the rat NAc shell, upon repeated passive administration of cocaine (Huang et al., 2009). These are primarily new synapses formed by insertion of GluN2B-containing NMDARs following in vivo cocaine exposure (Brown et al., 2011). This phenomenon explains, at least in part, the depression in glutamatergic transmission observed in the NAc directly after cocaine exposure (Thomas et al., 2001). It may also explain partially, the increase in spine density observed in MSNs of the NAc shell upon repeated treatment to cocaine (Robinson and Kolb, 1999), though there is no direct evidence for this link. However, the number of these silent synapses that are induced in the NAc shell upon exposure to cocaine, gradually decreases upon withdrawal from cocaine (Huang et al., 2009), whereas the increase in MSN spine density induced due to cocaine 
exposure is seen long after discontinuation of cocaine treatment (Robinson et al., 2001; Robinson and Kolb, 2004). This persistence of the structural plasticity is indicative of an unsilencing mechanism for these cocaine-induced silent synapses, during the period of withdrawal from cocaine. Thus, just as nascent silent synapses mature during early development, cocaine-induced silent synapses also have the potential to be unsilenced, thereby serving effectively as substrates for subsequent plasticity (hence a form of metaplasticity). It has been proposed that cocaine by generating silent synapses, can rejuvenate excitatory synapses in the NAc and prime them for subsequent strengthening during withdrawal from the drug, to form potentially durable drug-associated adaptive memories (Lee and Dong, 2011; Dong and Nestler, 2014a; Huang et al., 2015b).

The signaling scaffold protein PSD-95 has already been implicated to play a role in maturation of silent synapses during early development (Béïque et al., 2006; Huang et al., 2015a). It was also shown to have a role in psychostimulant action, because in mice chronically treated with cocaine, PSD-95 was downregulated as observed by a decrease in mRNA and protein levels in the striatum. In this study, behavioral alterations in mice carrying a targeted deletion of PSD-95 were also observed. These mice were overly sensitive to the acute stimulating effects of cocaine, as seen by an augmented locomotor response in the open field assay following acute administration of cocaine, but resistant to subsequent sensitization (Yao et al., 2004). Therefore PSD-95 through its role in synaptic maturation, may be involved in the expression of drug-dependent behavioral plasticity.

\subsubsection{CP-AMPAR expression and drug-related behavior}

In humans, relapse to cocaine-use after a prolonged period of withdrawal from the drug, often occurs due to drug-associated cues that provoke craving for the drug (O'brien et al., 1992). This cue-induced cocaine craving is thought to intensify during the first few weeks of withdrawal and stay elevated for prolonged periods of time (Gawin FH and Kleber HD, 1986). This has been modelled in rodents wherein, a time-dependent increase in cue-induced drug seeking, is observed during withdrawal from i.v cocaine-self administration and is termed as 'incubation of cocaine craving' (Grimm et al., 2001; Pickens et al., 2011). It was subsequently shown that it is the gradual accumulation of GluA2-lacking CP-AMPARs (calcium permeable AMPARs) in NAc neurons, during prolonged withdrawal from cocaine-self administration that mediates incubation of cocaine craving (Conrad et al., 2008; McCutcheon et al., 2011a). Therefore CP-AMPAR expression is considered to be a biomarker for incubation of craving, 
as the high conductance of CPAMPARs could increase the reactivity of NAc neurons to drugassociated cues. Not unlike the VTA (Bellone and Lüscher, 2006), this synaptic plasticity can be reversed in the NAc by activation of mGluR1, which results in removal of CP-AMPARs from synapses and hence suppression of incubation of craving (McCutcheon et al., 2011b; Loweth et al., 2014a, 2013a). Conversely, the blockade or antagonism of mGluR1 results in the intensification of incubation of craving (Halbout et al., 2014).

\subsection{Scope of the thesis}

The NAc plays a central role in the reward circuit of the brain by prioritizing emotional and motivational outputs for behavior. A new concept of the neural rejuvenation hypothesis for drug addiction is based on the finding that repeated cocaine exposure induces the generation of silent synapses in the NAc of adult animals (Huang et al., 2009). These silent synapses are thought to serve as plasticity substrates, primed for subsequent steps of plasticity, which eventually incorporates them in new neural connections. This step involves the incorporation of AMAPRs into these non-transmitting synapses, thereby switching them to transmitting synapses, which might present memory traces for drug-associated behaviors (Dong and Nestler, 2014b). The general goal of this study was to determine the fate of cocaine-induced silent synapses in the NAc and to assess their role in the long-term retention of drugassociated behavior.

The accumulation of CP-AMPARs in NAc synapses during withdrawal from the contingent procedure of cocaine self-administration, is a form of drug-induced synaptic plasticity that has been extensively studied in the past few years (Conrad et al., 2008; Lee et al., 2013; Loweth et al., 2014b; Ma et al., 2014). This process has been considered as a cellular marker for incubation of cocaine craving, the time dependent increase in cue-induced cocaine seeking that occurs during prolonged withdrawal from cocaine self-administration (Grimm et al., 2001; Pickens et al., 2011; Wolf and Tseng, 2012). However another study showed that passive cocaine exposure in mice is sufficient for the expression of CP-AMPARs in the NAc (Mameli et al., 2009), raising the possibility that CP-AMPARs could be a more general signature of drug experience. In this study I used a systematic approach to decipher the role of CPAMPARs in long-term retention of drug-related memory and incubation of craving. Moreover, I also addressed the molecular underpinnings of CP-AMPAR expression in NAc synapses and tested the requirement of a drug-paired association in this process. Therefore this study aimed to test whether cocaine-induced silent synapses in the NAc mature into CP-AMPAR- 
bearing synapses and if this maturation is required for long-term retention of drug-related memory.

Currently it is unknown how in vivo cocaine experience can generate silent synapses in adult NAc synapses. In this study I analyzed the involvement of thrombospondins, which are astrocyte-secreted synaptogenic proteins (Eroglu, 2009; Risher and Eroglu, 2012), in this process. By targeting the thrombospondin receptor a2ס1 (Eroglu et al., 2009), in NAc synapses of adult animals, I sought to find out whether a thromspondin- $\alpha 2 \delta 1$ pathway may be responsible for generation of silent synapses upon repeated cocaine exposure. 
2. Materials and Methods 


\section{Materials and Methods}

\subsection{Animal housing conditions}

Mice were kept under standard conditions of a 12 hour light/dark cycle with controlled temperature and humidity and were provided food and water ad libitum. All mice were weaned at 3 weeks of age after which males and females were separately housed. Wild type mice (BI6J background) were either bred in this facility or ordered from Charles River. Mice were always housed in groups of up to 4 animals per cage. All procedures were performed in the light cycle and were approved by the governmental agencies of the European Neuroscience Institute, Göttingen, Germany.

Rats were used for this study only at the University of Pittsburgh, PA, USA. Adult male Sprague-Dawley rats were order from Charles River and housed under standard conditions of $12 \mathrm{hr}$ light/dark cycle with controlled temperature and humidity. Rats were single housed post surgeries and provided food and water ad libitum. All procedures were performed in the light cycle and all experiments were in accordance with the protocols approved by the Institutional Animal Care and Use Committees at University of Pittsburgh.

\subsection{Cocaine treatment}

\subsubsection{Passive injection of cocaine in mice}

Wild type mice of at least 4 weeks of age, were administered intraperitoneal (i.p) injections of cocaine hydrochloride (Sigma, Germany) at a dosage of $20 \mathrm{mg} / \mathrm{kg}$ bodyweight and returned to their home cage. The control groups of animals were injected with $0.9 \% \mathrm{NaCl}$ saline (Braun, Germany), the vehicle in which the drug is prepared and returned to their home cage. Following 5 passive injections of cocaine/saline, one injection per day, the mice were sacrificed the next day ( 1 day of withdrawal from the drug) or after 20-30 days (long-term withdrawal from the drug) for electrophysiological measurements.

\subsubsection{Cocaine - Conditioned place preference (CPP) in mice}

Conditioned place preference assay was performed according to Roux and colleagues (Roux et al., 2003) with some minor modifications. Male wild type or SAP102 KO mice were used when they were at least 4-weeks of age at the start of the assay. During the first 3-5 days, the mice were allowed to acclimatize to the environmental conditions of the laboratory 
as well as to the handling of the experimenter for habituation. During this period, the mice were given sham intraperitoneal (i.p) injections once per day, to familiarize them to this procedure.

The CPP apparatus consisted of two main compartments, each $15 \mathrm{~cm} \mathrm{X} 15 \mathrm{~cm}$ in size having very distinct olfactory, visual and tactile cues. Each compartment was rendered with a different scent, either cinnamon or vanilla, by hosting a small tube stuffed with tissue paper dipped in scented oils. The walls and floor of each chamber were covered with distinct visual patterns, in the shape of black dots on a white background or black stripes on a white background. Furthermore, the floor of the chamber was designed to give tactile cues, either in the form of interspersed circular pits or interspersed long linear pits. These two conditioning compartments were separated by guillotine trap doors and a narrow, grey-colored corridor which served as a neutral area.

One day prior to conditioning and after the habituation phase, mice were placed the neutral area and allowed to freely explore both compartments for the next $20 \mathrm{~min}$. This was done to check the initial preference of the animal for either chamber, prior to conditioning. The preference for each compartment was calculated (see below) by making a video recording of the animal as it explored the apparatus. Animals which spent more than $75 \%$ of the time only in compartment (and less than $25 \%$ in the other) were removed from the study due their intrinsic bias.

CPP with cocaine was performed in an unbiased mode, wherein, animals were randomly assigned any one compartment for conditioning with cocaine. The conditioning or training lasted 10 days with alternating cocaine and saline injections. On the first day, each animal was given an i.p injection of cocaine hydrochloride (Sigma, Germany) at a dosage of 20 $\mathrm{mg} / \mathrm{kg}$ bodyweight and immediately placed in one chamber for the next $20 \mathrm{~min}$. The trap doors were closed all throughout the 10 days of conditioning, so that the animal was restricted to one compartment under the effect of the drug. The next day, the animal was given an i.p injection of the vehicle, $0.9 \% \mathrm{NaCl}$ saline (Braun, Germany) and placed in the second compartment. Therefore at the end of 10 days, the animals received 5 injections of cocaine in one compartment i.e. the conditioned compartment and 5 injections of saline in the other compartment.

1 day after the 10-day long training period, the trap doors were opened and the mice were placed in the neutral area such that they had free access to both compartments and their movement in the apparatus was monitored by video recording over the next 20 min. Subsequently, the time spent by the animal in each compartment was calculated from $18 \mathrm{~min}$ 
of the video recording using Anymaze software (Stoelting Co., USA). A place preference score (PPS) was calculated using the equation below -

$$
\text { PPS }=\frac{\text { Time spent in conditioned compartment }(\mathrm{sec}) \mathrm{X} \text { total time }(\mathrm{sec})}{\text { Total time }(\mathrm{sec}) \text {-Time in neutral area }(\mathrm{sec})}
$$

The animals were either sacrificed for electrophysiology directly after this test (at 1 day of withdrawal from the drug) or were kept for prolonged withdrawal from the drug, by housing them in their home cages for another 10-40 days, depending on the withdrawal time point to be studied. Thereafter, they were again tested to calculate a PPS for the conditioned chamber at a long-term withdrawal time point and subsequently sacrificed for electrophysiology.

\subsubsection{Intravenous Self-Administration (SA) of cocaine in rats}

The intravenous (i.v) self-administration procedure in rats as described before (Mu et al., 2010), necessitates the implantation of a silastic catheter into the right jugular vein. The catheters, approximately $8 \mathrm{~cm}$ in length were constructed from a silastic tubing (inner diameter 0.020 inches, outer diameter 0.037 inches; Helix Mark, USA). The surgery to implant the catheter was performed by Dr. Junshi Wang (Department of Neuroscience, University of Pittsburgh, PA, USA). Briefly, while the male rats (4-7 weeks old) were anesthetized with a mixture of Ketamine $(87 \mathrm{mg} / \mathrm{kg})$ and Xylazine $(13 \mathrm{mg} / \mathrm{kg})$, the catheter was implanted into the right jugular vein and the distal end was passed under the skin to an opening on the back between the scapulae and connected to a Quick Connect Harness (SAI Infusion). The incisions on the body were then sutured before the animal woke up from anesthesia. During recovery from this surgery, the catheter was flushed daily with $1 \mathrm{ml} / \mathrm{kg}$ heparin $(10 \mathrm{U} / \mathrm{ml})$ and gentamicin antibiotics $(5 \mathrm{mg} / \mathrm{ml})$ in sterile saline to protect against occlusion of the catheter and infections.

Cocaine SA procedure started 1-2 weeks post the catheter implantation, to allow the rats to recover. Operant-conditioning chambers enclosed within sound-attenuating cabinets (Med associates, USA) were used. Each chamber consisted of an active and inactive nose poke hole, a pump to deliver cocaine and a house light which was usually switched off. No food or water was provided for the rats during their sessions in these conditioning chambers. On the first day, rats were placed in the conditioning chambers for an overnight training session, 
wherein they had more time to acclimatize to the training conditions and learn the SA procedure. A nose poke by the rat into the active hole resulted in an intravenous cocaine infusion $(0.75 \mathrm{mg} / \mathrm{kg}$ over 3-6 sec; cocaine provided by National Institute on Drug Abuse Drug Supply Program, USA) from the pump into the catheter. Every active nose poke was also accompanied by a conditioned stimulus (CS) of illumination of blue light within that hole for 6 sec. Additionally, upon active nose poking by the rat, a the house light was also switched on for a period of $20 \mathrm{sec}$ during which active nose pokes were counted but did not result in any cocaine infusions, to avoid overdosing. After the $20 \mathrm{sec}$ time-out period, the house light was turned off and the next active nose poke would result in a cocaine infusion in a similar fashion. Nose poking in the inactive hole had none of these consequences, but was nevertheless recorded. Rats were allowed to take a maximum of 100 cocaine infusions during the overnight session and only those which took at least 40 cocaine infusions were used for the subsequent 5-day SA training. After the overnight session and a gap of one day, the 5day SA training regimen consisted of $2 \mathrm{hr}$ sessions per day with the same conditions as that used for overnight training. Control animals received no infusions (sham) or saline infusions.

Incubation of cocaine craving was measured by performing a $1 \mathrm{hr}$ extinction test at 1 day withdrawal from cocaine SA (next day after the 5-day long training) or at 40 days of withdrawal from cocaine SA (long-term withdrawal during which rats were simply housed in their home cages). During the extinction tests, the active nose pokes by the rat were accompanied by the contingent light cues but resulted in no cocaine infusions. The number of active nose pokes before and after withdrawal was compared to assess incubation of cocaine craving.

\subsection{Positive allosteric modulation of mGluR1}

For the activation of mGlur1-LTD a selective positive allosteric modulator (PAM) of mGluR1 called SYN119 was used for systemic injections in mice (Ngomba et al., 2011; Loweth et al., 2014b). This compound is relatively insoluble in aqueous solutions, therefore a surfactant solution of $10 \%$ Tween 80 dissolved in $0.9 \% \mathrm{NaCl}$ saline (Braun, Germany) was used as the vehicle to deliver SYN119 to mice via the i.p route. A fine suspension of SYN119 in $10 \%$ Tween 80 could be prepared by brief sonication using a sonicator probe, followed by continuous vortexing. Immediately prior to every i.p injection of Syn119, the suspension was warmed to $37^{\circ} \mathrm{C}$ and vortexed vigorously before loading into syringes. The conditioned place preference (CPP) protocol was same as described above and the effect of Syn119 was 
studied only after long-term withdrawal from cocaine-CPP. The mice were given one i.p injection of Syn119 $(20 \mathrm{mg} / \mathrm{kg})$ at 20-30days of withdrawal from cocaine and left undisturbed in their home cage for the next $1 \mathrm{hr}$. They were then placed in the CPP apparatus and tested as usual to determine their place preference score (PPS) post the SYN119 treatment. Immediately following this 20 min long test, the animals were sacrificed and processed for electrophysiology. The control animals were treated only with the vehicle $10 \%$ Tween 80 and subsequently processed in the identical manner.

\subsection{Genotyping}

The SAP102 KO (knock-out) mouse used in this study was generated by Cuthbert and colleagues (2007). The genotype was determined by a specific PCR (Polymerase Chain Reaction; (Saiki et al., 1985)) performed on genomic DNA samples isolated from small tail pieces of the mice. 1-3 mm long tails tips were cut from mice at approximately 3 weeks of age during weaning from the mother. These tail pieces were lysed in PBND buffer (see below) supplemented with $1: 100$ Proteinase $\mathrm{K}$ solution (Ambion $20 \mathrm{mg} / \mathrm{ml}$ ) at $55^{\circ} \mathrm{C}$ with constant vigorous shaking for at least 2 hours. The dissolved tail samples were then heated to $99^{\circ} \mathrm{C}$ for $10 \mathrm{~min}$ in order to inactivate the enzyme Proteinase $\mathrm{K}$ present in the lysis buffer. These DNA samples could be stored at $4^{\circ} \mathrm{C}$ on a short-term basis and were used for the following PCR reaction -

\section{PCR mixture $\quad$ PCR Program}

$2 \mu \mathrm{l} \quad$ Sample

$2.2 \mu \mathrm{l} \quad$ 10x TNK Buffer

$2 \mu \mathrm{l} \quad \mathrm{dNTPs}(2.5 \mathrm{mM}$ each, Bioline)

$0.2 \mu \mathrm{l} \quad 50 \mu \mathrm{M}$ Primer 1

$0.2 \mu \mathrm{l} \quad 50 \mu \mathrm{M}$ Primer 2

$0.2 \mu \mathrm{l} \quad 50 \mu \mathrm{M}$ Primer 3

$15 \mu \mathrm{l} \quad \mathrm{H}_{2} \mathrm{O}$

$0.2 \mu \mathrm{l}$ Mango Taq DNA polymerase

(Bioline)
Time temperature

Pre-incubation $\quad 5^{\prime} \quad 94^{\circ} \mathrm{C}$

Denaturation $\quad 30^{\prime \prime} \quad 94^{\circ} \mathrm{C}$

Annealing $\left.\quad 30^{\prime \prime} 56^{\circ} \mathrm{C}\right\} \times 33$

Elongation $\quad 1^{\prime} \quad 72^{\circ} \mathrm{C}$

Final Elongation $10^{\prime} \quad 72^{\circ} \mathrm{C}$ 
Solutions and primers used for genotyping:

PBND lysis buffer $-10 \mathrm{mM}$ Tris, $50 \mathrm{mM} \mathrm{KCl}, 2.5 \mathrm{mM} \mathrm{MgCl}_{2} .6 \mathrm{H}_{2} 0,0.1 \mathrm{mg} / \mathrm{ml}$ gelatin, $0.45 \%$ (v/v) Nonident P40, 0.45\% (v/v/ Tween 20, $\mathrm{pH} 8.3$.

10X TNK buffer - $100 \mathrm{mM}$ Tris, $15 \mathrm{mM} \mathrm{MgCl}_{2}, 500 \mathrm{mM} \mathrm{KCl}, 50 \mathrm{mM} \mathrm{NH}_{4} \mathrm{Cl}$

Primer 1 sequence - GGTCTCTGATGAAGCAGTGATTTTT

Primer 2 sequence - TGATGACCCATAGACAGTAGGATCA

Primer 3 sequence - CTAAAGCGCATGCTCCAGAC

The PCR reaction mix was run on a $1 \%$ agarose gel in sodium tetraborate buffer $(5 \mathrm{mM}$


at $130 \mathrm{~V}$ for approximately $30 \mathrm{~min}$. The PCR products were visualized on a UV-illuminator with INTAS imaging system. Using the above primers, the wild-type allele is seen as a 535bp DNA band while the KO allele is seen as a 215 bp DNA band.

\subsection{Virus Production}

\subsubsection{Large scale plasmid preparation}

\subsubsection{Plasmid DNA used}

A shRNA (short hairpin RNA) construct for RNAi-mediated knockdown of endogenous PSD-95 protein (targeting the PSD-95 sequence GGA CAT CCA GGC ACA CAA G), called 'sh95' was prepared as described previously (Schlüter et al., 2006). This construct under the control of a $\mathrm{H} 1$ promoter cassette from pSuper vector, was cloned into an AAV vector backbone containing an EGFP expression marker under the control of a CAG promoter.

A shRNA construct for RNAi-mediated knockdown of $\alpha 2 \delta 1$ (or A2D1) protein (targeting the a2ס1 sequence GCTGAGTTAGAGAATGAAATT), called 'bITPd' was prepared similarly and cloned into a FUGW lentivirus backbone (Lois et al., 2002) wherein bITPd was under the control of $\mathrm{H} 1$ promoter cassette and the expression marker EGFP was under the control of a Ubiquitin promoter.

The experimental controls were either the empty vector backbone bearing the EGFP expression marker or those which additionally contained a shRNA targeted to the luciferase gene (sequence targeted-CCTAAGGTTAAGTCGCCCTCG), called suLC, as this gene is not present in the rodent genome. 


\subsubsection{DNA Maxi Prep}

The DNA plasmids were transformed into the electro-competent SURE bacteria (Stratagene, CA, USA) using electroporation at $1800 \mathrm{~V}$, with a time constant between 4-6 ms (Eppendorf 2510 electroporator). The electroporated bacteria were spread onto LB-Agar plates containing ampicillin (100 $\mu \mathrm{g} / \mathrm{ml}$, Roth, Germany) and allowed to grow for 12-16 hrs at $37^{\circ} \mathrm{C}$. A single colony of transformed bacteria was then picked from the plate and used for inoculating $50 \mathrm{ml}$ LB-medium (Roth, Germany) which was allowed to grow at $37^{\circ} \mathrm{C}$ for $12-16$ hrs at constant shaking of 350rpm (INNOVA 4230, Brunswick Scientific shaker). The bacterial cells were then harvested by $4000 \mathrm{rpm}$ centrifugation at $4^{\circ} \mathrm{C}$ for $30 \mathrm{~min}$ (Eppendorf Centrifuge 5804R with rotor A4-44) and subjected to standard alkaline lysis using the Qiagen (Hilden, Germany) solutions P1, P2 and P3 (see below) according to the Qiagen protocol (Birnboim and Doly, 1979). Following alkaline lysis, DNA was precipitated with 0.7X volumes of isopropanol (AppliChem) and resuspended in $600 \mu \mathrm{l}$ of buffer TE $(10 \mathrm{mM}$ Tris- $\mathrm{HCl}, 10 \mathrm{mM}$ EDTA pH 8.5). The residual RNA was removed by incubation of the DNA along with $1 \mu \mathrm{l}$ RNase $A$ and $150 \mu \mathrm{l} 1 \mathrm{M} \mathrm{NaCl}$ at $50^{\circ} \mathrm{C}$ for $5 \mathrm{~min}$. The proteins were removed from the DNA sample by phenol/chloroform extraction. The sample was mixed with an equal volume of 25:24:1 phenol: chloroform: isoamyl alcohol (Applichem) in Phase Lock Gel tubes (Eppendorf) and vortexed vigorously. The phases were separated by centrifugation at full speed for 1 min (Eppendorf 5424 table top centrifuge) after which the upper aqueous phase was transferred to a new tube and mixed again with an equal volume of chloroform and again centrifuged, for removal of the residual phenol. The aqueous phase was subsequently collected and used for precipitating DNA with $0.7 \mathrm{X}$ volumes of isopropanol. The precipitated pellet was washed with $70 \% \mathrm{EtOH}$ and finally suspended in $200 \mu \mathrm{l}$ of EB (Elution Buffer $10 \mathrm{mM}$ Tris-HCl, pH 8.5; Qiagen). In order to purify the DNA further, it was precipitated by addition of $1 \mathrm{ml} 100 \%$ Ethanol, $100 \mu \mathrm{l} 1 \mathrm{M} \mathrm{NaCl}$ and $200 \mu \mathrm{H}_{2} 0$ followed by vortexing and centrifugation at top speed for $15 \mathrm{~min}$ at room temperature (RT). The supernatant was aspirated carefully and the pellet was dried at RT before finally resuspending it in 300-400 $\mu \mathrm{l} E B$. The DNA was kept at $-20^{\circ} \mathrm{C}$ for long term storage.

Solutions used for DNA preparation:

Buffer P1 - 50mM Tris-HCl pH 8.0, 10mM EDTA 100 $\mu \mathrm{g} / \mathrm{ml}$ RNase A

Buffer P2 - 200mM NaOH, 1\%SDS w/v

Buffer P3 - 3.0M Potassium acetate pH 5.5 


\subsubsection{HEK cell culture}

HEK 293T cells - Human embryonic kidney cells, expressing large T-antigen (Graham et al., 1977) were used to produce lentiviral and adeno-associated viral vectors. These cells were cultured in Dubelco's high glucose Dulbecco's Modified Eagle's Medium (DMEM; Biochrom) supplemented with 10\% fetal bovine serum (FBS; Biochrom). The cells were grown on $10 \mathrm{~cm}$ dishes at $37^{\circ} \mathrm{C}$ and $5 \% \mathrm{CO}_{2}$ and routinely passaged by washing with phosphate buffer saline (PBS; Biochrom) followed by standard trypsin (Biochrom) dissociation (Masters and Stacey, 2007).

\subsubsection{Adeno Associated Virus (AAV) production}

\subsubsection{HEK 293T cell transfection for AAV8 preparation}

HEK 293T cells were transfected using polyethyleneimine (PEI) following a procedure modified from Kuroda and colleagues (Kuroda et al., 2009). The transfection reagent was prepared by mixing $2.88 \mathrm{ml}$ of $15 \mathrm{mM}$ linear PEI (Polysciences) with $24 \mathrm{ml} 150 \mathrm{mM} \mathrm{NaCl}$ and allowed to incubate for $10 \mathrm{~min}$ at RT. In a separate tube $24 \mathrm{ml}$ of $150 \mathrm{mM} \mathrm{NaCl}$ was mixed with $300 \mu \mathrm{g}$ pDP8ape (helper plasmid for AAV8 serotype; PlasmidFactory) and 300 $\mu \mathrm{g}$ of the vector plasmid and left to incubate for $10 \mathrm{~min}$ at RT. The transfection reagent was then added to the prepared DNA mixture, mixed thoroughly by vortexing and left to incubate for $10 \mathrm{~min}$ at RT. Meanwhile HEK 293T cells from 10X 15cm dishes (Greiner) which were $70-80 \%$ confluent were harvested by trypsinization, pelleted down by centrifugation at $700 \mathrm{~g}$ for $5 \mathrm{mins}$ at RT and resuspended in 240ml DMEM medium (with glutamine; Biochrom). The PEI/DNA mixture was then added to the HEK cell suspension and mixed gently before plating it onto $15 \times 15 \mathrm{~cm}$ dishes, $19 \mathrm{ml}$ per dish. These dishes were then returned to $37^{\circ} \mathrm{C}$ and $5 \% \mathrm{CO}_{2}$ and harvested after 72 hours.

\subsubsection{Harvesting and purification of AAV particles}

The medium from the transfected $15 \mathrm{X} 15 \mathrm{~cm}$ dishes was removed and replaced with $3 \mathrm{ml}$ PBS per dish. The AAV was then harvested by scraping the dish surface with a $25 \mathrm{~cm}$ cell scraper (Sarstedt) and centrifuging the pooled cells at $1000 \mathrm{~g}$ for $10 \mathrm{~min}$ at RT. The cell pellet was then resuspended in $8.5 \mathrm{ml}$ of $20 \mathrm{mM}$ Tris, $150 \mathrm{mM} \mathrm{NaCl}$ at pH8.0. For cell lysis $500 \mu \mathrm{l}$ $10 \% \mathrm{NaDOC}$ (see below) was added (final concentration $0.5 \%$ ) followed by addition of a nuclease $-2 \mu \mathrm{l}$ Benzonase (250U/ $\mu \mathrm{l}$; Novagen) or $10 \mu \mathrm{l}$ Cyanase $(50 \mathrm{U} / \mu \mathrm{l})$, thorough mixing and incubation at $37^{\circ} \mathrm{C}$ for $30 \mathrm{~min}$ (with occasional vigorous shaking). $584 \mathrm{mg} \mathrm{NaCl}$ was then 
dissolved in this solution and kept for another 30 min of incubation at $56^{\circ} \mathrm{C}$. The tube was cooled down at ice for $5 \mathrm{~min}$ and then frozen and stored overnight at $-80^{\circ} \mathrm{C}$.

For purification of the AAV sample, a discontinuous iodixanol gradient was prepared in a U-shaped ultracentrifuge tube, using a 20G cannula needle (Dispomed, $0.9 \times 70 \mathrm{~mm}$ ) attached to a $10 \mathrm{ml}$ syringe. The gradient was prepared by diluting a $60 \%$ iodixanol stock solution in 1X PBS-MK (see below). The gradient was as follows - layers from top to bottom $6.5 \mathrm{ml} 15 \%$ iodixanol, $4 \mathrm{ml} 25 \%$ iodixanol, $3 \mathrm{ml} 40 \%$ iodixanol and $3 \mathrm{ml} 54 \%$ iodixanol. The topmost layer was loaded first at the bottom of the tube, and the successive heavier layers were carefully added to the bottom one by one. The AAV sample was thawed at $37^{\circ} \mathrm{C}$ for the purification procedure and lysed cell debris was removed by centrifugation at $4000 \mathrm{~g}$ for 30 min at RT. The supernatant was carefully loaded onto the discontinuous iodixanol gradient and then centrifuged for $1.5 \mathrm{hr}$ at 60,000 rpm in an ultracentrifuge (Beckman L8-60M with rotor $70 \mathrm{Ti}$ ). AAV particles get collected in the $40 \%$ iodixanol layer, therefore this layer was collected by carefully placing the $20 \mathrm{G}$ cannula at the $40 \%-54 \%$ interphase and sucking it off gently with the syringe. This solution containing AAV particles was then diluted with 1X PBSMK solution (see below) to up to $10 \mathrm{ml}$ and concentrated in a $100 \mathrm{~K}$ cell concentrator (Amicon) by centrifugation at $2000 \mathrm{~g} \mathrm{RT}$. This concentrating step was repeated thrice and the concentrated virus particles were diluted with $1 \times$ PBS-MK up to $500 \mu \mathrm{l}$. After filtration through a $0.22 \mu \mathrm{m}$ syringe driven filter unit (Millex-GV), the purified AAV was stored at $4^{\circ} \mathrm{C}$.

Solutions used for harvesting and purifying AAV particles:

$10 \% \mathrm{NaDOC}-10 \mathrm{~g}$ sodium salt of deoxycholic acid dissolved in $100 \mathrm{ml}$ water and filter sterilized $(0.22 \mu \mathrm{m}$ filter $)$

10X PBS-MK - $0.2 \mathrm{~g} \mathrm{MgCl}_{2} \cdot 6 \mathrm{H}_{2} \mathrm{O}$ (Merck) and $0.19 \mathrm{~g} \mathrm{KCl}$ (Sigma) dissolved in $100 \mathrm{ml} 10 \mathrm{X}$ PBS and filter sterilized $(0.22 \mu \mathrm{m}$ filter $)$

\subsubsection{Extra-large scale lentivirus preparation}

\subsubsection{HEK 293T cell transfection for lentivirus preparation}

Lentiviral particles were produced by transfecting HEK 293T cells with the plasmid DNA of interest, HIV-1 packaging vector $\Delta 8.9$ and envelope glycoprotein vector vs.vg. Polyethyleneimine ( $\mathrm{PEI}$ ) was used as the transfection reagent following a procedure modified from Kuroda and colleagues (Kuroda et al., 2009). $1.835 \mu \mathrm{g}$ PEI solution (branched; SigmaAldrich) was mixed with $20 \mathrm{ml}$ Opti Pro SFM-Medium (without Glutamine; Life Technologies) and allowed to incubate for $10 \mathrm{~min}$ at RT. In a separate tube $270 \mu \mathrm{g}$ of the desired DNA 
plasmid, $135 \mu \mathrm{g}$ vs.vg vector and $202.5 \mu \mathrm{g} \Delta 8.9$ vector was mixed in $5 \mathrm{ml}$ Opti Pro SFMMedium (without Glutamine) and left to incubate for $10 \mathrm{~min}$ at RT. The transfection reagent was then added to the prepared DNA mixture, mixed thoroughly by vortexing and left to incubate for $30 \mathrm{~min}$ at RT. $70-80 \%$ confluent, $9 \times 15 \mathrm{~cm}$ dishes of HEK 293T cells were used for transfection. $3 \mathrm{ml}$ of the DNA-PEI mixture was added to each plate, following which the plates were returned to $37^{\circ} \mathrm{C}$ and $5 \% \mathrm{CO}_{2}$ for up $4-5$ hours. The medium was then carefully changed completely to $20 \mathrm{ml}$ OptiPro SFM-Medium containing $2 \mathrm{mM}$ Glutamine, and the plates were returned to $37^{\circ} \mathrm{C}$ and $5 \% \mathrm{CO}_{2}$ and harvested after 48 hours.

\subsubsection{Harvesting and purification of lentiviral particles}

The supernatant from all the transfected plates was pooled and centrifuged at $2000 \mathrm{rpm}$ for $10 \mathrm{~min}$. The supernatant was then filtered through the membrane of a $0.45 \mu \mathrm{m}$ Stericup filter (HV Durapore Membran, Millipore). The collected flow-through was distributed into ultracentrifuge tubes ( $25 \times 89 \mathrm{~mm}$, Beckmann) and a cushion of $3 \mathrm{ml} 20 \%$ sucrose was added below the supernatant. The weight of all the ultracentrifuge tubes was balanced on an analytical scale, before ultracentrifugation at $25000 \mathrm{rpm}$ (Bekmann L8-60M with rotor 70Ti) at $4^{\circ} \mathrm{C}$ for 2 hours. The supernatant and sucrose was then carefully poured off and the walls of the tubes dried with tissue paper in order to avoid dilution of the sample. The pellet of lentiviral particles was resuspended in $75 \mu \mathrm{l}$ PBS by gentle swirling and trituration and then left overnight at $4^{\circ} \mathrm{C}$ for further dissolution. The residual debris was removed by centrifugation at $3000 \mathrm{rpm}$ at RT for 2 min (Eppendorf 5424 table top centrifuge) and the supernantant containing concentrated lentivirus was aliquoted and kept for long-term storage at $-80^{\circ} \mathrm{C}$.

\subsection{Stereotaxic viral injections in rodents}

\subsubsection{Stereotaxic viral injections in mice}

2-3 days prior to the stereotactic surgery, 4-week old mice were given the painkiller Metamizol (Tropfen $1.5 \mathrm{mg} / \mathrm{ml}$, Ratiopharm) in drinking water, which was changed to normal drinking water 1 week post the surgery. On the day of the surgery, the mice were anesthetized using a mixture of Fentanyl $(0.07 \mathrm{mg} 7 \mathrm{~kg}$; Rotexmedica), Medetomedine (7.0 $\mathrm{mg} / \mathrm{kg}$; CP Pharma) and Midazolam (0.7 mg/kg; Ratiopharm) via i.p injection, and fixed on a stereotactic frame (Stoelting). An incision was made on the head of animal in order to access the skull. The head was then aligned using the bregma and lambda (the intersection point of cranial sutures used routinely for stereotactic surgery). If needed, small doses of anesthesia 
were injected as boosters via the subcutaneous (s.c) route. Small holes were drilled through the skull at the desired coordinates, using a drill mounted on the stereotactic frame (OmniDrill35, World Precision Instruments). Approximately 0.7-1 $\mu \mathrm{l}$ (depending on the virus titer) of the AAV8 virus was then injected bilaterally through the drilled holes, into the nucleus accumbens (NAc) using a glass micropipette attached to a Drummond Nanoject II injector and a Micro4 microsyringe pump controller (World Precision Instruments) at the rate of 0.14 $\mu \mathrm{l} / \mathrm{min}$. During the surgery, a bolus of warm saline was given via an S.c injection to avoid hypothermia and dehydration. The needle was left in place for $5 \mathrm{~min}$ following the viral infusion and then slowly retracted. The head incision was then sutured and the anesthesia was antagonized by s.c injections of $1 \mathrm{mg} / \mathrm{kg}$ Atipamazole (Antisedan, Orion pharma) and 0.1 $\mathrm{mg} / \mathrm{kg}$ Buprenorphine (Temgesic, Reckitt Benckiser). Additionally, an s.c injection of $5 \mathrm{mg} / \mathrm{kg}$ Carprofen (Rimadyl, Pfizer $\mathrm{GmbH}$ ) was given as an analgesic. The mice were allowed to recover for at least 2 weeks prior to commencing any procedure as this also provides sufficient time for AAV8 transduction and expression in the NAc neurons.

The stereotaxic coordinates used for mouse NAc: anterior-posterior (AP), + 1.55; mediallateral (ML), \pm 0.55 ; dorsal-ventral (DV), -4.70 .

\subsubsection{Stereotaxic viral injections in rats}

Stereotaxic injections in rats to be used for cocaine i.v self-admininstration were performed at Department of Neuroscience, University of Pittsburgh, PA, USA. The stereotaxic surgery was performed 1 week prior to the catheter implantation surgery or immediately after the catheter implantation with only one dose of anesthesia. In both cases, rats (4-7 weeks old) were anesthetized with a mixture of Ketamine $(87 \mathrm{mg} / \mathrm{kg})$ and Xylazine $(13 \mathrm{mg} / \mathrm{kg})$ administered via i.p injection and then placed on a heating pad to avoid hypothermia. The rats were administered the painkiller ketoprofen intramuscularly (i.m) in the thigh muscle, before starting the surgery. Similar to the stereotaxic injection in mice, the rats were then fixed in a stereotactic frame (Stoelting) and after making an incision on the head of the animal, the head was aligned using the bregma and lambda. If needed, small doses of anesthesia were injected as boosters via the i.m route. Small holes were drilled through the skull at the desired coordinates, using a hand held drill. Approximately 1.1-1.4 $\mu \mathrm{l}$ of the AAV8 solution was then injected bilaterally through the drilled holes into the NAc using a 23 gauge injection needle attached to a Hamilton syringe and a Thermo Orion M365 pump at a rate of $0.22 \mu \mathrm{l} / \mathrm{min}$. A bolus of $1 \mathrm{ml}$ saline was given to the anesthetized rats via s.c injection to avoid dehydration. 
Similar to the procedure described for mice, the needle was left in place for 5 min following the viral infusion after which it was slowly retracted, the head incision was sutured and the animals were allowed to recover from the anesthesia. After waking up from the anesthesia, the rats were returned to their individual home cages for 2 weeks.

The stereotaxic coordinates used for rat NAc: anterior-posterior (AP), + 1.50; medial-lateral (ML), \pm 0.85 ; dorsal-ventral (DV), - 7.25.

\subsubsection{Imaging of virus injection site}

The site of viral infusion was checked for several injected mice at the electrophysiology microscope by observing the expression of the marker GFP. Images were taken using IC Capture software, to document the exact injection site and spread of the virus expression. For the sole purpose of imaging, some injected mice which were used for the CPP behavior assay, were not used for electrophysiology but were instead perfused transcardially with cold $50 \mathrm{ml} 0.01 \mathrm{M}$ PBS followed by cold $75 \mathrm{ml} 4 \%$ paraformaldehyde $-4 \%$ sucrose under gravity flow. All injected rats which were used for cocaine-SA, were perfused transcardially, while blocking the descending aorta, with cold 50ml 0.01M PBS followed by cold $200 \mathrm{ml} \mathrm{4 \%}$ paraformaldehyde - 4\% sucrose using a pump (performed at Department of Neuroscience, University of Pittsburgh, PA USA). Following perfusion the mice/rat brain was dissected out and kept in cold $4 \%$ paraformaldehyde - $4 \%$ sucrose overnight for post-perfusion fixation. The brains were then transferred to 0.1M PBS and 200-300 $\mu \mathrm{m}$ thick coronal slices of both brain hemispheres were cut using a vibratome (Leica Vibratome1200S). The brain slices were then stained with DAPI (1:200 dilution of a $1 \mathrm{mg} / \mathrm{ml}$ stock solution) for at least $15 \mathrm{~min}$ and washed twice with $0.01 \mathrm{M}$ PBS before mounting onto glass slides using Mowiol (Calbiochem) supplemented with the anti-fading agent DABCO (2.5\% w/v 1,4,-diazobicycli-[2.2.2]octane, Aldrich). After the mounting solution was allowed to set at $-20^{\circ} \mathrm{C}$, the slices were imaged using a stereomicroscope (Nikon) with a 1X SHR Plan Apo objective. Higher magnification images were obtained using a confocal microscope (Zeiss LSM 710, $40 \mathrm{X}$ objective). 


\subsection{Electrophysiology}

\subsubsection{Preparation of acute brain slices of the NAc}

For preparation of acute brain slices, the animals were anesthetized with isoflurane $(100 \%$ Forene, Abbvie) and the mice were decapitated directly. Rats were however perfused transcardially with $50 \mathrm{ml}$ ice-cold NMDG cutting buffer (bubbling with $5 \% \mathrm{CO}_{2}$; see below) using a pump, prior to decapitation in order to allow the significantly larger rat brain to cool down. The rest of the procedure remains the same for both mice and rats although slightly different tools were used to dissect the harder and tougher skull of the rat brain.

The brain was isolated by dissection of the head and immediately submerged in ice-cold NMDG cutting buffer for maximum 5 minutes. $300 \mu \mathrm{m}$ thick coronal slices of both brain hemispheres were cut using a vibratome (Leica Vibratome1200S). The slices containing nucleus accumbens (NAc) were collected and transferred to ACSF (artificial cerebrospinal fluid, see below) at $35^{\circ} \mathrm{C}$ for $10-15 \mathrm{~min}$, for recovery with continuous bubbling with $5 \% \mathrm{CO}_{2}$. The slices, submerged in ACSF were then kept at RT for at least 30 min before starting electrophysiological recordings.

Solutions used for acute slice preparation -

NMDG cutting buffer - $135 \mathrm{mM}$ NMDG (N-methyl D-glucamine, Sigma), $1 \mathrm{mM} \mathrm{KCl}$ (Sigma), $1.2 \mathrm{mM} \mathrm{KH}_{2} \mathrm{PO}_{4}, 1.5 \mathrm{mM} \mathrm{MgCl}_{2} \cdot 6 \mathrm{H}_{2} \mathrm{O}$ (Sigma), $0.5 \mathrm{mM} \mathrm{CaCl} \cdot 2 \cdot \mathrm{H} 2 \mathrm{O}, 10 \mathrm{mM} \mathrm{D}$ Glucose (Merck), and $20 \mathrm{mM}$ choline bicarbonate (Sigma) .

ACSF recovery buffer - $119 \mathrm{mM} \mathrm{NaCl}$ (Roth), $2.5 \mathrm{mM} \mathrm{KCl}$ (Sigma), $1 \mathrm{mM} \mathrm{NaH}_{2} \mathrm{PO}_{4}$

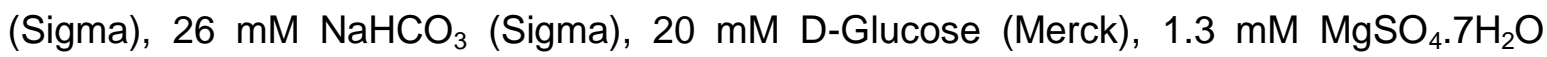
(Roth) and $2.5 \mathrm{mM} \mathrm{CaCl}_{2} \cdot 2 \mathrm{H}_{2} \mathrm{O}$ (Roth).

The above solutions were freshly prepared in the above working concentrations and were continuously bubbled with carbogen gas $\left(95 \% \mathrm{O}_{2}\right.$ and $\left.5 \% \mathrm{CO}_{2}\right)$.

\subsubsection{Electrophysiology setup}

All electrophysiology recordings were done using the following setup -

- ZEISS AXIO Examiner D.1 Microscope with 5X A-Plan air objective and 63X W PlanAPOCHROME water objective

- ELC-03XS amplifier (NPI instruments, Germany)

- ITC-18 data acquisition board (HEKA) 
- Micromanipulator SM-5 (Luigs \& Neumann, Germany)

- ISO-FLEX stimulator (A.M.P.I, Jerusalem, Israel)

- Hamamatsu CCD camera

- TC-20 Temperature controller (NPI instruments, Germany)

- Igor Pro 6.2 - custom programmed data acquisition software (Wave Metrics)

The external solution ACSF (see below), was bubbled continuously with $5 \% \mathrm{CO}_{2}$ in its reservoir and was made to perfuse through the recording chamber at a constant rate of 2-3 $\mathrm{ml} / \mathrm{min}$, using a peristaltic pump. The recording chamber and incoming ACSF was also heated to maintain a temperature between $27-30^{\circ} \mathrm{C}$. The patch pipettes made of borosilicate glass (KG-33, King Precision Glass Inc, USA) were prepared using a micropipette puller (P-1000 and Sutter Instruments) in order to have a tip of 1-2 $\mu \mathrm{m}$ diameter with 3-5 $\mathrm{M} \Omega$ resistance. The patch pipette was filled with internal solution (see below) and approached to the cells of interest, identified visually on a monitor. Whole-cell patch clamp recordings were carried out in voltage-clamp configuration, with signals being filtered at $3 \mathrm{kHz}$. Series resistance and input resistance were monitored throughout the experiment. Cells with unstable or high $(\geq \sim 40 \mathrm{~m} \Omega)$ series resistance were discarded from the experiment.

Solutions used for acute slice preparation -

External solution (ACSF) - $119 \mathrm{mM} \mathrm{NaCl}$ (Roth), $2.5 \mathrm{mM} \mathrm{KCl}$ (Sigma), $1 \mathrm{mM} \mathrm{NaH}{ }_{2} \mathrm{PO}_{4}$ (Sigma), $26 \mathrm{mM} \mathrm{NaHCO}_{3}$ (Sigma), $20 \mathrm{mM}$ D-Glucose (Merck), $1.3 \mathrm{mM} \mathrm{MgSO} 4.7 \mathrm{H}_{2} \mathrm{O}$ (Roth), 2. $5 \mathrm{mM} \mathrm{CaCl}_{2} .2 \mathrm{H}_{2} \mathrm{O}$ (Roth). Freshly prepared and kept bubbling with $5 \% \mathrm{CO}_{2}$ at RT.

Internal Solution - 50\% w/v CsOH (Sigma), $117.5 \mathrm{mM} \mathrm{MeSO}_{3} \mathrm{H}$ (Fluka), $10 \mathrm{mM} \mathrm{HEPES}$ (Roth), $17.75 \mathrm{mM} \mathrm{CsCl}$ (Roth), $10 \mathrm{mM}$ TEA-Cl (Fluka), 0.25 mM EGTA (Roth), $10 \mathrm{mM}$ Glucose (Merck), 2 mM MgCl $2.6 \mathrm{H}_{2} \mathrm{O}$ (Sigma), $5 \mathrm{mM} \mathrm{QX-314} \mathrm{chloride} \mathrm{(Sigma),} 4 \mathrm{mM}$ $\mathrm{Na}_{2} \mathrm{ATP}$ (Sigma) and 0.3 mM NaGTP (Sigma). pH was adjusted to 7.0-7.3 using $\mathrm{CsOH}$ and osmolarity was adjusted to $\sim 290 \mathrm{mOsm}$. The solution was stored at $-80^{\circ} \mathrm{C}$ and kept at $4^{\circ} \mathrm{C}$ while in use. 


\subsubsection{Electrophysiological recordings from the NAc shell}

All electrophysiological recordings were performed on medium spiny neurons (MSNs) of the NAc shell which were visually identified and selected at random. Local interneurons present in the same region were much lesser likely to be chosen since they constitute $<5 \%$ of the neuronal population. However, if selected, interneurons were identified by their distinct properties such as high input resistance, high spontaneous activity and low NMDA responses and removed from the data set. Excitatory postsynaptic currents (EPSCs) were evoked from medium spiny neurons (MSNs) of the NAc using electrical stimulation. A borosilicate 2-barrel glass capillary (World Precision Instruments) filled with external solution which was placed in the NAc shell tissue, was used as the stimulator. Additionally, the external solution (ACSF) contained $100 \mu \mathrm{M}$ Picrotoxin (GABA $\mathrm{A}$ receptor blocker; Abcam/Ascent Scientific, Germany) to block inhibitory currents.

The MSNs were patched in whole-cell configuration at a holding potential of $-60 \mathrm{mV}$. AMPA EPSCs were recorded at this voltage and upon depolarizing the cell by slowly changing the holding potential to $+40 \mathrm{mV}$, a composite of AMPA and NMDA EPSCs could be recorded. Two main electrophysiological assays used in this study are described below.

\subsubsection{Failure Analysis - Quantification of proportion of silent synapses}

Failure analysis following a minimal stimulation protocol was used as a method to count the percentage of silent synapses among total synapses, in the recorded neuron (Huang et al., 2009). The intensity of electrical stimulation, which was done at a frequency of $0.2 \mathrm{~Hz}$, was first adjusted to obtain small AMPA EPSCs $(<40 \mathrm{pA})$ and then further reduced in increments such that failures (no responses) and successes (small responses) could be distinguished visually. The stimulation frequency and strength were then kept constant throughout the experiment, even after depolarization to $+40 \mathrm{mV}$. Thus the stimulation intensity is adjusted to obtain both failures and small responses at $-60 \mathrm{mV}$ and $+40 \mathrm{mV}$. $\sim 50$ sweeps in total were recorded at each holding potential while intermittently switching between the two potentials (at least 2 times). The number of failures and responses were then counted manually and the 
percentage silent synapse composition was calculated according to the following equation below wherein $F_{-60 m v}$ and $F_{+40 m v}$ refers to the failure rate at $-60 \mathrm{mV}$ and $+40 \mathrm{mV}$ respectively.

$$
\% \text { Silent synapses }=1-\frac{\operatorname{Ln}\left(F_{-60 m v}\right)}{\operatorname{Ln}\left(F_{+40 m v}\right)}
$$

\subsubsection{AMPAR Rectification Index - Quantification of proportion of CP- AMPARs}

In order to isolate pure AMPA EPSCs, also at depolarized potentials, $50 \mu \mathrm{M}$ D-AP5 (or APV; Ascent, UK/Abcam, Germany), an NMDAR blocker, was added to the external ACSF solution. Additionally $100 \mu \mathrm{M}$ of Spermine (polybasic amine for voltage-dependent block of CP-AMPARs; Roth, Germany) was added to the internal solution. EPSCs were evoked at a stimulation frequency of $0.1 \mathrm{~Hz}$ and the stimulation intensity was adjusted to obtain EPSC amplitudes between 50-350 pA. 30 stable sweeps were recorded at $-60 \mathrm{mV}$, the cell was then depolarized and $\sim 5$ sweeps at $0 \mathrm{mV}$ (equilibrium potential of AMPARs) were recorded, followed by another $\sim 30$ sweeps at $+40 \mathrm{mV}$. The amplitude of AMPAR response at $-60 \mathrm{mV}$ (AMPA EPSC $_{-60 \mathrm{mv}}$ ) and $+40 \mathrm{mV}\left(\right.$ AMPA EPSC $_{+40 \mathrm{mV}}$ ) was calculated from the peak of the response in reference to the baseline. The rectification index is calculated using the following ratio.

$$
\text { Rectification Index }=\frac{\text { AMPA EPSC }_{-60 \mathrm{mV}}}{\text { AMPA EPSC }_{+40 \mathrm{mV}}}
$$

\subsection{Data Analysis}

All results are represented as mean values \pm SEM (standard error of mean).

$$
\mathrm{SEM}=\frac{\sigma}{\sqrt{n}} \quad \sigma \text { is the standard deviation and } \mathrm{n} \text { is the sample size }
$$

Data from repeated runs of the same experiment was pooled and statistical analysis was done using Student t-test or and one-way ANOVA followed by post-hoc Bonferroni test. The significance in the data has been indicated as: ${ }^{*} p<0.05 ;{ }^{* *} p<0.01$ and ${ }^{* *} p<0.001$. 
The sample size $(n)$ for data from animal behavior experiments is indicated by $n=$ no. of animals tested.

For data from electrophysiological recordings, each condition is indicated by $n / m=$ Number of animals / number of cells sampled. For all electrophysiological data animal based statistics was done by averaging the values from all cells recorded in a single animal for a particular assay. 
3. Results 


\section{Results}

\subsection{Cocaine-conditioned place preference in wild type mice and long-term retention of drug-related memory}

In wild type mice I performed cocaine-conditioned place preference (CPP), a behavioral assay used to test a positive association with the drug (for details refer to section 1.4.1). After being accustomed to the laboratory conditions and handling procedures, the mice were placed in the CPP apparatus. This consisted of two chambers bearing distinct visual, olfactory and tactile cues (with dots or stripes for the sake of simplicity) separated by a neutral zone. In the initial 'pre-test' the mice were allowed to explore both chambers for 20 min and a place preference score (PPS) was calculated to determine the baseline preference of the mice for either chamber (for details refer to section 2.2.2). For each mouse, one of the two chambers was selected for being paired with the drug-experience. On the first day of the CPP training, each mouse was administered cocaine via i.p injection and immediately placed in the selected cocaine-paired chamber only (for example stripes in Figure 3.1A) for the next $20 \mathrm{~min}$. On the next day the mouse was injected with the vehicle control saline and placed into the opposite chamber (dots in case of Figure 3.1A). This procedure was repeated over 10 days with alternating cocaine and saline injections wherein the mouse associates the experience of the drug cocaine only in one chamber and not the other. On the $11^{\text {th }}$ day, considered as withdrawal day 1 (WD1), the mouse was placed into the neutral zone of the apparatus without any injection and was allowed to explore both chambers. The time spent in each chamber was monitored during this test and the PPS for the selected cocaine-paired chamber was calculated. An increase in the PPS for the cocaine-paired chamber compared to the baseline PPS, is considered as a development of a 'preference' for the cocaine-paired chamber. It indicates that during the CPP training, the mouse learned the association of the rewarding experience of cocaine to its specific environment and therefore spent more time in the selected cocaine-paired chamber, even in the absence of the drug.

As a control, some mice were injected only with saline throughout the CPP training and as expected they did not develop a preference for any chamber (baseline to WD1: PPS $521.7 \pm$ 22.78 to $491.2 \pm 24.83 ; n=28$; paired t-test $p=0.219$; Figure $3.1 \mathrm{~B}$ ). With cocaine however wild type mice developed a high preference for the cocaine-paired chamber as shown by an increase in the PPS (baseline to WD 1: PPS $529.26 \pm 21.55$ to $716.77 \pm 16.26 ; n=29$; paired t-test $p<0.001$; Figure $3.1 B)$. For different cohorts of mice, I tested them again after a defined 
period of withdrawal, wherein the mice were left undisturbed in their home cages, to see if they still remember the preference that was developed by the CPP training. The CPP test after prolonged withdrawal was performed in a manner identical to that on WD 1(Figure 3.1 A) and a PPS score higher than the baseline score calculated from the pre-test indicated that the animal remembered the preference, as it still spent more time in the cocaine-paired chamber. For the cohorts tested for 10-15 days of withdrawal from cocaine, the mice learnt and remembered the preference for the cocaine paired chamber (baseline to WD 1 to WD 10: PPS $529.45 \pm 23.10$ to $661.24 \pm 19.45$ to $614.51 \pm 22.13 ; n=21$; paired t-test, pre-test PPS vs. WD1 PPS $p<0.001$; paired t-test, pre-test PPS vs. WD10 PPS $p<0.01$ ). Similarly the cohorts tested at 20-25 days of withdrawal from cocaine had also learnt and remembered the preference for the cocaine-paired chamber (baseline to WD1 to WD 20: PPS $538.15 \pm 23.12$ to $710.97 \pm 16.06$ to $729.97 \pm 21.39 ; n=25$; paired t-test, pre-test PPS vs. WD1 PPS $p<0.001$; paired t-test, pre-test PPS vs. WD20 PPS $p<0.001$ ). The mice tested at $30-35$ days of withdrawal also remembered the preference they had learnt (baseline to WD 1 to WD 30: PPS $511.85 \pm 27.11$ to $690.24 \pm 15.79$ to $628.62 \pm 25.98$; $n=27$; paired t-test, pre-test PPS vs. WD1 PPS $p<0.001$; paired t-test, pre-test PPS vs. WD30 PPS $p<0.01$ ) and so did the ones tested at 40-45 days of withdrawal (baseline to WD 1 to WD 40: PPS $549.62 \pm 25.08$ to $736.14 \pm 24.88$ to $745.20 \pm 27.30 ; n=20$; paired t-test, pre-test PPS vs. WD1 PPS $p<0.001$; paired t-test, pre-test PPS vs. WD40 PPS $p<0.001$ ). Therefore these results show that the memory of drug-associated cues developed by the cocaine-CPP training is robust and lasts till at least 45 days of withdrawal from the drug. 
A
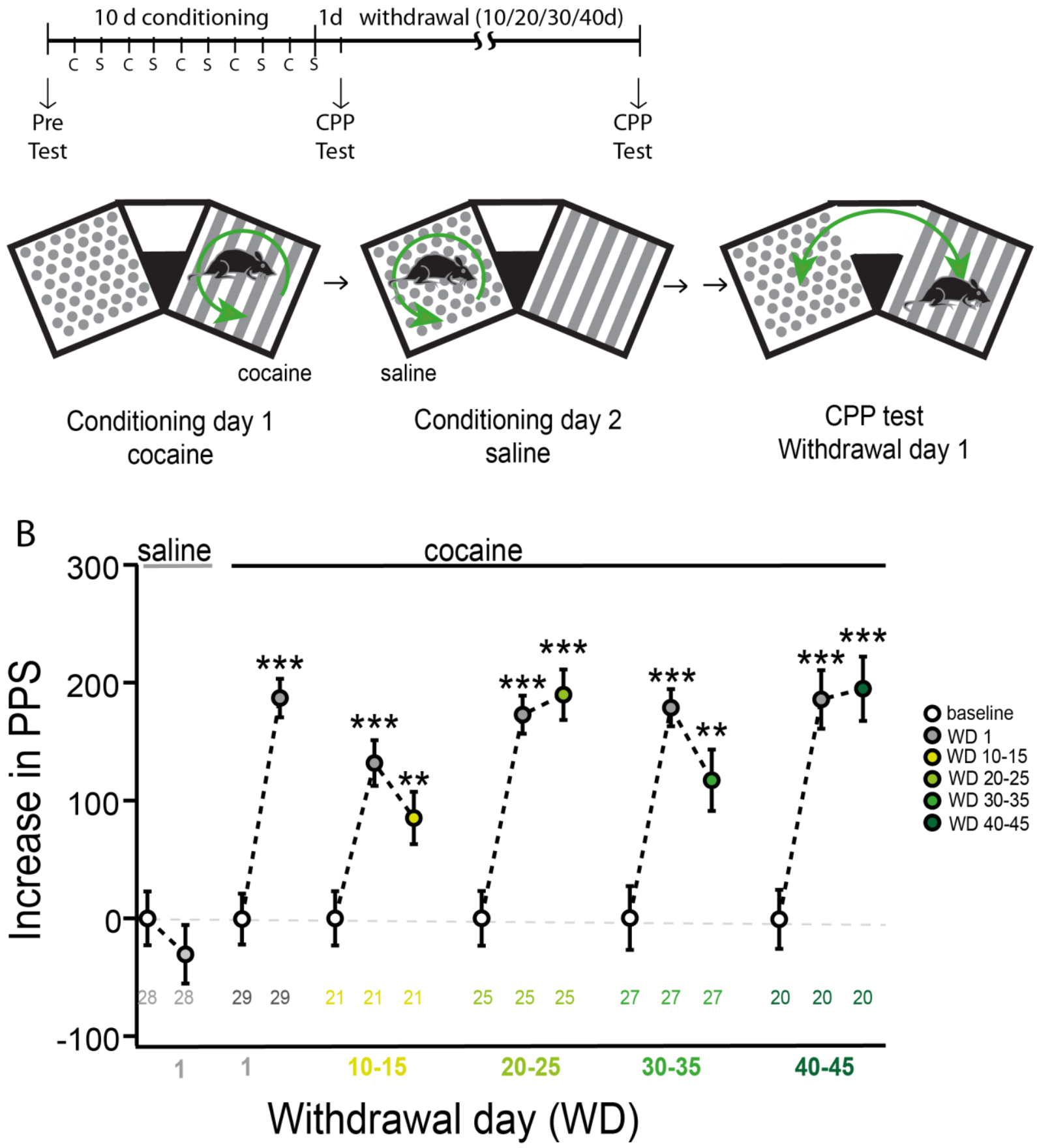

Figure 3.1 Wild type mice learn and remember the association with the cocaine-paired chamber in the CPP paradigm, up till $\mathbf{4 5}$ days after last cocaine injection.

(A) Timeline of the CPP experiment with training (C-cocaine, S-saline) and withdrawal days indicated along with a schematic representation of the training and testing days of the assay. (B) The increase in the place preference score (PPS) is indicated by normalizing to the baseline score before commencement of the training. When trained with saline, wild type mice do not develop a preference whereas with cocaine they do. The preference was shown to be present after long term withdrawal from cocaine. Number of animals used ( $n$ ) is indicated below each group. Statistical significance was assessed using paired t-test. Some of the experiments were done in collaboration with A.Suska (see section 5 appendix, for details). 


\subsection{Increase in number of silent synapses in the nucleus accumbens shell upon cocaine exposure and accumulation of calcium permeable AMPARs during withdrawal from cocaine}

At the cellular level, I focused on the nucleus accumbens (NAc), a brain region considered as a critical target of actions of cocaine and other drugs of abuse. It is an essential part of the reward circuit wherein experience-dependent plasticity has been thought to mediate, at least in part, the durable memory underlying cocaine addiction (Hyman et al., 2006a). The NAc is divided into two major sub regions, the NAc shell (NAcSh) and the NAc core, that are part of distinct neuroanatomical circuits and have been implicated in distinct aspects of drug-seeking behavior (Ito et al., 2004). Intravenous cocaine has been shown to preferentially increase extracellular dopamine levels in the nucleus accumbens shell (NAcSh) (Pontieri et al., 1995; Rodd-Henricks et al., 2002). Moreover, the activity of NAcSh neurons indirectly influences the NAc core. Therefore, for the sake of consistency, all the electrophysiological experiments described in this thesis were conducted from neurons of NAcSh. Medium spiny neurons (MSNs), which are the principle cell type in this region of the brain, comprise of $>95 \%$ of the local neuronal population and constitute the output of NAc, while the rest consists of interneurons of several types. All electrophysiological recordings analyzed in this study were performed on MSNs of the NAcSh chosen at random, by visual identifying them and thereafter confirming their identity by their electrophysiological properties.

After the CPP behavior testing (section 3.1), some of the wild type mice were used for electrophysiology recordings from the NAcSh MSNs. The mice were sacrificed for preparation of acute slices either directly after the CPP tests on WD1 or after the last CPP test post prolonged withdrawal from cocaine (figure 3.2A). For failure analysis, the minimal stimulation protocol (Huang et al., 2009) was used (figure 3.2C-E) such that either small EPSCs (responses, shown in black) were evoked or failures (shown in grey) were produced from the cell being held at a holding potential of $-60 \mathrm{mV}$ and $+40 \mathrm{mV}$, sequentially. The proportion of silent synapses was then estimated wherein, a higher failure rate at $-60 \mathrm{mV}$ indicates a higher proportion of silent synapses in the recorded cell (for details refer to section 2.7.3.1). The baseline proportion of silent synapses in the NAcSh MSNs was determined in mice that were treated only with the vehicle control, saline during the CPP training. At withdrawal day 1 (WD 1 ) these mice had an average of $24.13 \% \pm 3.83 \%$ silent synapses in the NAc MSNs (white 
circle, figure $3.2 \mathrm{~F} ; \mathrm{n} / \mathrm{m}=12 / 46$ ). The mice subjected to cocaine-CPP however had $46.90 \% \pm$ $3.01 \%$ silent synapses at WD 1, indicating almost a two fold increase in number of silent synapses upon cocaine exposure (grey circle, figure 3.2F; $\mathrm{n} / \mathrm{m}=14 / 42$; post hoc Bonferroni test after one-way ANOVA (see below) saline vs. WD 1, $p<0.001$ ). Using the mice cohorts which were kept for defined periods of long-term withdrawal after cocaine-CPP, the time course of these cocaine-induced silent synapses was measured. It was observed that silent synapse number reduces by WD 10-15 and falls back to baseline level by WD 20-25 (figure 3.2F light to dark green circles: WD 10-15, 35.60\% $\pm 4.20 \%, \mathrm{n} / \mathrm{m}=9 / 29$; WD 20-25, $19.61 \%$ $\pm 4.87 \%, n / m=6 / 22 ;$ WD 30-35, 22.73\% $\pm 5.01 \%, n / m=8 / 14 ;$ WD $40-45,23.32 \% \pm 4.45 \%$, $\mathrm{n} / \mathrm{m}=7 / 16$; one-way ANOVA for all conditions $F_{5,51}=7.57$, p $<0.001$, post hoc Bonferroni test saline vs. WD 1, $p<0.001)$. These results show that silent synapses induced due to cocaine exposure mature to non-silent synapses during withdrawal from cocaine.

Accumulation of CP-AMPARs into the NAc synapses has been shown to occur during long-term withdrawal from cocaine self-administration and is thought to be a biomarker for incubation of craving (Conrad et al., 2008; Grimm et al., 2001; McCutcheon et al., 2011a; Pickens et al., 2011). I sought to find out whether CP-AMPARs could accumulate in the NAcSh synapses after cocaine-CPP and if this process is a relatively more general signature of drug-related behaviors. To estimate the proportion of GluA2 lacking, calcium permeable AMPA receptors (CP-AMPARs) in the NAcSh following cocaine-CPP, the rectification index was recorded from MSNs (for details refer to section 2.7.3.2) wherein AMPA EPSCs were evoked at holding potentials of $-60 \mathrm{mV}, 0 \mathrm{mV}$ and $+40 \mathrm{mV}$ sequentially (figure $3.2 \mathrm{~B}$ ). A higher rectification index (AMPA EPSC $-60 \mathrm{mv} /$ AMPA EPSC $_{+40 \mathrm{mv}}$ ) implies a higher proportion of CPAMPARs in the recorded neuron. The baseline rectification index was calculated after salineCPP at WD 1 and was observed to be $1.99 \pm 0.11$ (figure $3.2 F$ white square; $n / m=9 / 34$ ). At WD1 following cocaine CPP, the rectification index was not different from baseline (1.96 \pm 0.08 , grey square Figure $3.2 F ; n / m=13 / 39$ ). However the rectification index increases when measured after long-term withdrawal from cocaine-CPP. By WD 20-25 the rectification index was higher than baseline and it peaked during WD 30-35 after which it again decreased (figure 3.2F light to dark green squares: WD 10-15, $2.14 \pm 0.17, \mathrm{n} / \mathrm{m}=7 / 23$; cocaine WD 20$252.45 \pm 0.12, \mathrm{n} / \mathrm{m}=6 / 23$; cocaine WD 30-35, $2.74 \pm 0.16 ; \mathrm{n} / \mathrm{m}=7 / 21$; cocaine WD 40-45 $2.29 \pm 0.11, \mathrm{n} / \mathrm{m}=10 / 40$; one-way ANOVA for all conditions $F_{5,48}=5.34, p<0.001$, post hoc Bonferroni test saline vs. WD $30 p<0.001$; t-test saline vs. WD $20 p<0.05$; t-test WD 30 vs. WD $40 p<0.05)$. These results show that CP-AMPARs accumulate slowly in NAcSh synapses during prolonged withdrawal from cocaine-CPP. Previously it has been reported that only 
contingent drug-delivery procedures such as cocaine self-administration can induce accumulation of CP-AMPARs in the NAc and not experimenter-administered procedures (McCutcheon et al., 2011a). Therefore these results prove that experimenter-administered procedures such as passive i.p injection of cocaine during a CPP paradigm can also lead to accumulation of CP-AMPARs in the NAcSh.

Furthermore, the complementary kinetics of silent synapse maturation and CP-AMPAR accumulation during withdrawal from cocaine-CPP suggests that the silent synapses induced by cocaine-CPP might be unsilenced or matured by incorporation of CP-AMPARs to form specialized synaptic connections in the context of drug-associated behavior (Lee et al., 2013).

A

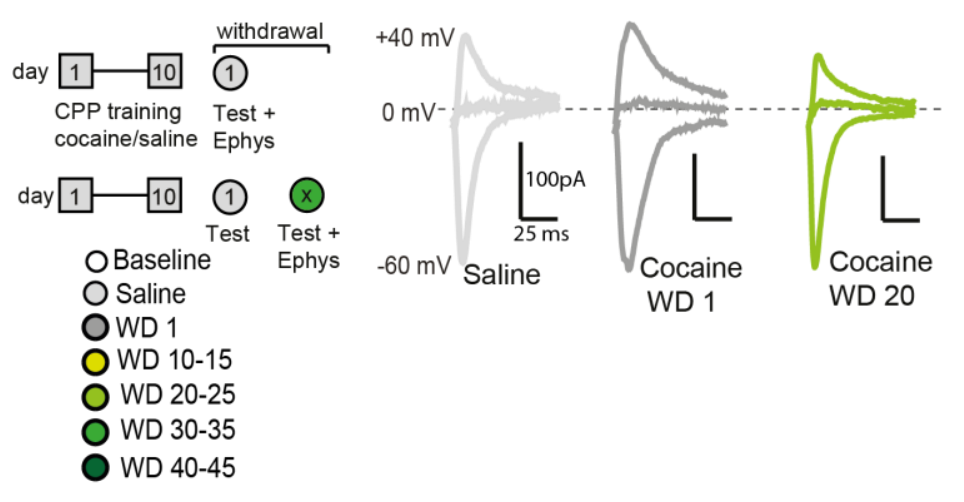

C
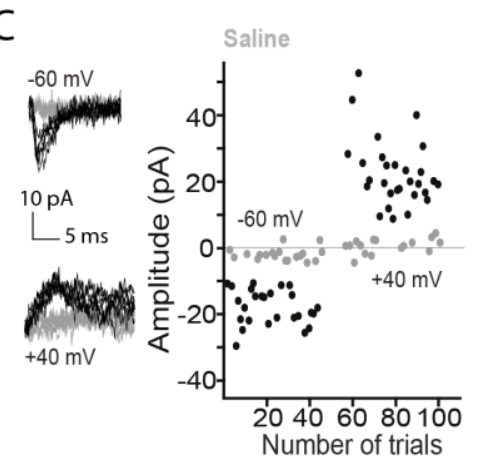
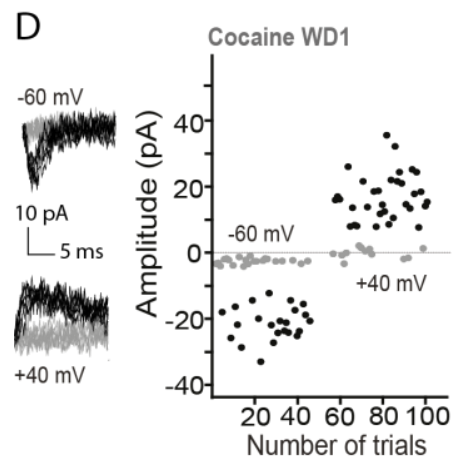

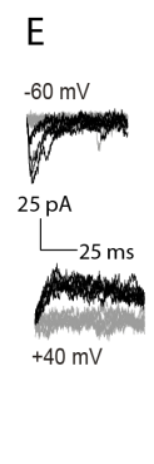

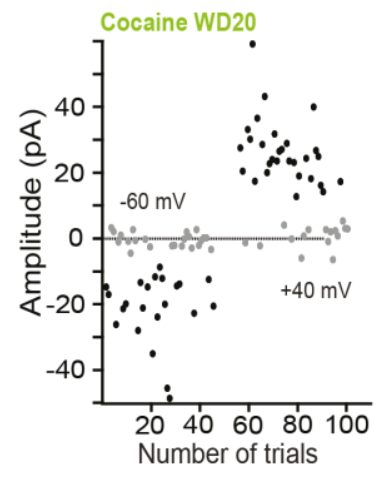

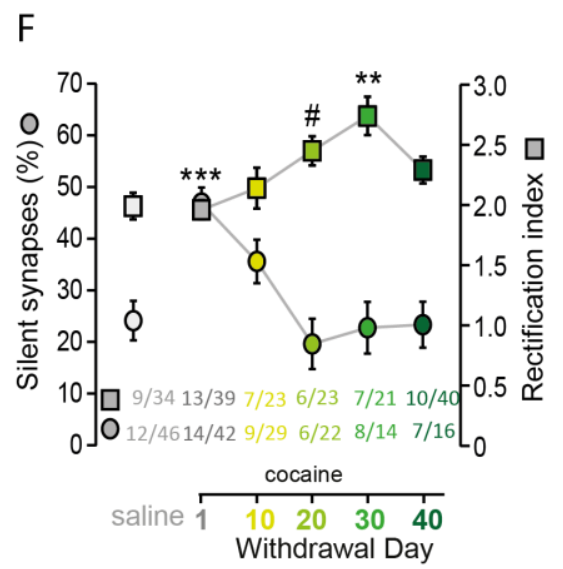

Figure 3.2 Electrophysiological assays performed in the cells of the nucleus accumbens shell (NAcSh) show that withdrawal from cocaine-CPP is accompanied by a decrease in number of silent synapses and accumulation of calcium-permeable rectifying AMPARs.

Scheme followed for electrophysiology on the animals used for CPP in Figure 3.1. (B) Example AMPA EPSCs evoked at $-60 \mathrm{mV}, 0 \mathrm{mV}$ and $+40 \mathrm{mV}$ during rectification index assay. (C), (D), (E) - Example recordings of EPSCs evoked by minimum stimulation 1 day after saline-CPP (C), cocaine-CPP (D) or 20-25 days post cocaine-CPP (E). (F) Proportion of silent synapses in the NAcSh, increases dramatically 1 day following cocaine-CPP compared to the baseline observed by saline-CPP and then 
decreases back to baseline during withdrawal. Rectification index, a measure of number of calcium permeable AMPARs (CP-AMPARs) in NAcSh synapses increases slowly during withdrawal, reaching a peak at WD 30-35 before returning to baseline. Number of animals/number of cells sampled $(\mathrm{n} / \mathrm{m})$ is indicated in the figure below the respective data points. Some of the experiments were done in collaboration with A.Suska (see section 5 appendix, for details).

\subsubsection{The positive association learnt during cocaine-CPP and withdrawal from cocaine is necessary for accumulation of CP. AMPARs into the NAcSh synapses}

The results mentioned above, show that CP-AMPARs can accumulate in NAcSh synapses during withdrawal from cocaine-CPP (see above), in spite of it being a non-contingent behavioral paradigm. In previous studies, CP-AMPARs were shown to accumulate in the NAc only during withdrawal from contingent behavioral paradigms such as cocaine-selfadministration. They have been long considered as a hallmark for increase in cue-dependent drug seeking, in such operant conditioning protocols, known as incubation of craving (Conrad et al., 2008; McCutcheon et al., 2011a). However, it has also been shown in that 10 daily passive injections of cocaine in 4-6 week old mice, can increase the rectifying index in the NAc neurons after 35 days of withdrawal from cocaine, thereby indicating incorporation of CP-AMPARs (Mameli et al., 2009). This is in contrast to the finding by McCutcheon et al., 2011 that in adult rats, CP-AMPARs only accumulate in the NAc upon withdrawal from selfadministration of cocaine and NOT experimenter-administration of cocaine. Therefore it was interesting to decipher what can induce accumulation of CP-AMPARs in the NAcSh by a behavioral paradigm involving experimenter-administered cocaine. Hence some control experiments were performed, which are elucidated below.

Since passive injections of cocaine have been shown to NOT induce CP-AMPAR accumulation (McCutcheon et al., 2011a) it would suggest that the association learnt during the CPP assay was critically needed for this plasticity. To test this, wild type mice were given alternating cocaine or saline injections for 10 days (like in the case of cocaine-CPP) only in their home cage and were never exposed to the CPP apparatus (Figure 3.3A orange). When subjected to 20 days of withdrawal from cocaine, the rectification index in NAcSh was found to be same as baseline (figure 3.3B,C orange; home cage cocaine WD 20, $1.95 \pm 0.04, \mathrm{n} / \mathrm{m}=$ 5/20; post hoc Bonferroni test saline-CPP WD 1 vs. home cage cocaine WD 20, p>0.05). This result indicates that CP-AMPARs accumulate in the NAcSh synapses after passive cocaine injections only if the animal learns to associate the cocaine experience with particular stimuli such as the cocaine-paired chamber of the CPP assay. Cocaine injections given in the home cage, in the absence of any other learnt association, is not enough to induce accumulation of 
CP-AMPAR in NAcSh synapses. Therefore these results demonstrate that this plasticity is specific to association learnt during the cocaine-CPP training.

It was also essential to test whether CP-AMPARs accumulate slowly during withdrawal, or are induced by exposure to drug-related cues while conducting the final CPP-test before the electrophysiology recordings. To test this, we decided to exclude the step of CPP test which exposes the animal to cocaine-associated cues after 20 days of withdrawal from cocaineCPP. Thus the usual CPP protocol was followed, except that after 20 days of withdrawal the animals were used directly for electrophysiological recordings, without conducting any behavior test with the CPP apparatus prior to that (figure 3.3A, red). This resulted in high rectification index compared to baseline (figure 3.3B, C red; without cue-exposure WD20 2.41 \pm 0.15 ; one-way ANOVA for all conditions, $F_{3,19}=3.13, p<0.05$; post hoc Bonferroni test saline-CPP WD 1 vs. without cue exposure WD 20, $p<0.05$ ). Therefore, this shows that $C P$ AMPARs accumulate in NAcSh synapse already after prolonged withdrawal from cocaineCPP and are NOT induced on a smaller time scale in response to the cocaine-associated cues. This result also makes it possible to use the same mice for electrophysiology that were used in the CPP behavior experiment.

I also asked whether withdrawal from cocaine-CPP is absolutely required or could time after the first drug exposure be causal for inducing CP-AMPAR accumulation in NAcSh synapses? Since CP-AMPARs were seen to be highest around 20-35 days of withdrawal from cocaine-CPP (Figure 3.2F), I extended the CPP-training which usually lasts for 10 days, till 30 days (Figure 3.3A, yellow). Rectification index measured from NAcSh MSNs at one day of withdrawal from 30-day long cocaine-CPP, was same as the baseline measured from animals that underwent saline-CPP for 10 days (Figure 3.3C, yellow; saline-CPP WD 1, 1.99 $\pm 0.11, \mathrm{n} / \mathrm{m}=9 / 34 ; 30 \mathrm{~d}$ cocaine-CPP WD 1, $2.00 \pm 0.13, \mathrm{n} / \mathrm{m}=3 / 9$; saline; post hoc Bonferroni test saline-CPP WD 1 vs. $30 \mathrm{~d}$ cocaine-CPP WD $1 p>0.05$ ). From this result it can be concluded that CP-AMPARs do NOT accumulate in NAcSh synapses simply over time due to exposure from cocaine. Instead, a prolonged period of withdrawal from cocaine is absolutely required for this. Hence higher rectification index or CP-AMPAR accumulation in NAcSh MSNs is a signature of changes induced due to withdrawal from the drug cocaine.

For the sake of comparison, the data of wild type mice subjected to saline-CPP and used for electrophysiology on WD1 (grey and same as figure 3.2C) has been shown again. The NAcSh MSNs of the saline-CPP condition were recorded as interleaved controls throughout the course of the experiments. They have been pooled as so significant difference was observed for cohorts of animals tested at different time points. 
A

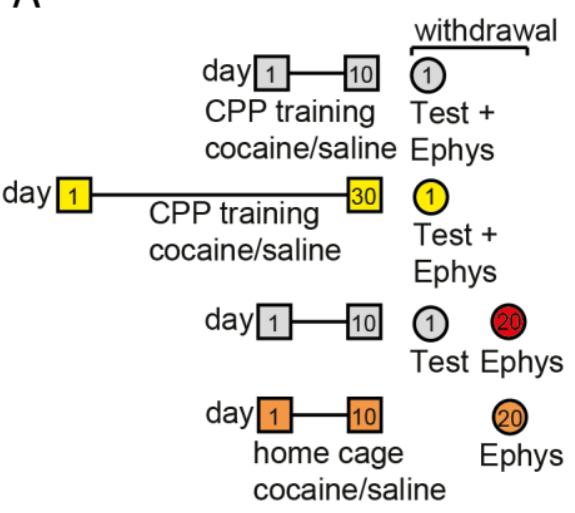

B

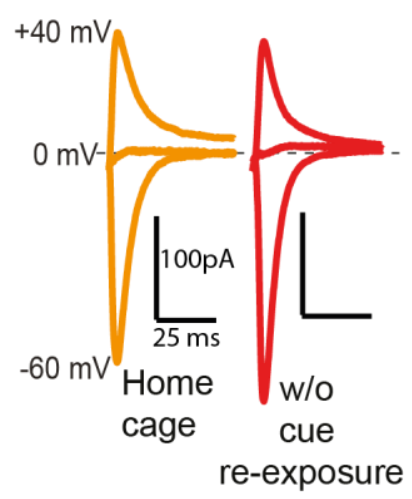

C

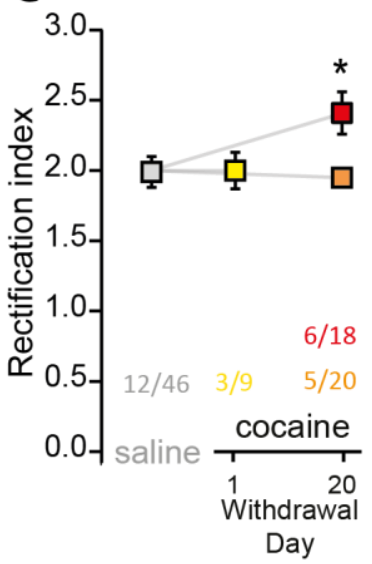

Figure 3.3 Calcium-permeable AMPARs accumulate during withdrawal from cocaine only when the association is learnt in the cocaine-CPP assay.

(A) Scheme for control experiments performed to check the specificity of CP-AMPARs accumulation in NAc synapses following cocaine exposure. (B) Example AMPA EPSCs evoked at $-60 \mathrm{mV}, 0 \mathrm{mV}$ and $+40 \mathrm{mV}$ during rectification index assay. (C) Cocaine injections in the home cage only, resulted in baseline rectification (orange) showing that increase in rectification is dependent on the association learned during cocaine-CPP. The behavior test performed on the mice just prior to electrophysiology does not induce accumulation of CP-AMPARs as significantly high rectification could be seen when this test was omitted (red). The 30-day long CPP protocol resulted in baseline level of rectification (yellow), showing that withdrawal from cocaine is necessary to observe an increase in rectification index. The saline control at 1 day of withdrawal from saline-CPP (same as shown in Fig.3.2C) has been used an interleaved control. $\mathrm{n} / \mathrm{m}$ is indicated in the figure below the respective data points. These experiments were done in collaboration with A.Suska (see section 5 appendix, for details).

\section{3 mGluR1 activation re-silences NAcSh synapses by removal of CP-AMPARs}

The complementary kinetics of silent synapse maturation and CP-AMPAR accumulation in NAcSh synapses, upon withdrawal from cocaine-CPP, suggests that silent synapses might be maturing by accumulating CP-AMPARs. To test this, we used an approach to remove CPAMPARs from synapses after withdrawal from cocaine-CPP and see whether the number of silent synapses increases upon doing so. The latter would suggest that CP-AMPARs in fact accumulate into the very same silent synapses that were induced upon cocaine exposure.

A recent study has shown that a systemic i.p injection of a mGluR1 positive allosteric modulator (PAM) called SYN119 can transiently attenuate the high rectification which is usually observed in the NAc of rats after prolonged withdrawal from cocaine selfadministration (Loweth et al., 2014a). Although the exact mechanism has not been studied in detail, SYN119 was shown to block CP-AMPAR transmission in rats by allosterically 
enhancing mGluR1 receptor function and causing the selective removal of CP-AMPARs from synapses through a mGluR-LTD like mechanism (Loweth et al., 2013b). I therefore decided to use SYN119 systemic injection on wild type mice which were in withdrawal from cocaineCPP for 20-30 days, a time point when rectification index was seen to be higher than baseline (figure 3.2F). The mice were trained for the cocaine-CPP behavioral assay as usual and left undisturbed in their home cage after testing them on WD 1, to ensure that they had learnt the association of the cocaine-paired chamber. After 20-30 days, the mice were given an i.p injection of $20 \mathrm{mg} / \mathrm{kg} \mathrm{SYN119}$ and after $1 \mathrm{hr}$ they were used for the CPP behavior test to check their long-term retention of the cocaine-CPP associated memory (Figure 3.4A). Thereafter they were directly processed for electrophysiology. As a control, mice trained for cocaine-CPP were injected with same volume of vehicle solution (10\% Tween 80 was used as the vehicle for SYN119) and then processed in the identical manner.

In the NAcSh slices of SYN119-treated animals, the rectification index (Figure 3.4C) was significantly lower as compared to the vehicle controls (figure 3.4F squares; saline WD1 grey, $1.99 \pm 0.11, \mathrm{n} / \mathrm{m}=9 / 34$; cocaine + vehicle injection black WD 20-30, $2.69 \pm 0.12, \mathrm{n} / \mathrm{m}=5 / 14$; cocaine + SYN119 injection WD 20-30, $2.10 \pm 0.13, \mathrm{n} / \mathrm{m}=9 / 23$; one-way ANOVA $\mathrm{F}_{2,20}=$ 7.121, $\mathrm{p}<0.01)$. Since the vehicle-injected controls had a high rectification compared to the saline WD1 baseline (Figure 3.4F squares, saline WD 1 grey vs. cocaine + vehicle injection black WD 20-30, post hoc Bonferroni test $p<0.01$ ) the administration of an i.p injection 20-30 days after withdrawal from cocaine-CPP, does not impair the accumulation of CP-AMPARs in the NAcSh synapses. The rectification index was significantly lower for SYN119-treated animals compared to vehicle controls, at the same time point of withdrawal from cocaine-CPP (vehicle injection black WD 20-30 vs. cocaine + SYN119 injection WD 20-30, post hoc Bonferroni test $p<0.05)$. This implies that one systemic injection of SYN119 was sufficient to remove CP-AMPARs that would have accumulated in the NAcSh synapses due to prolonged withdrawal from cocaine-CPP. Since SYN119 worked effectively with this protocol, I also performed the minimum stimulation protocol on NAcSh MSNs to estimate the proportion of silent synapses (figure 3.4D,E). SYN-119 treated animals in fact, had higher proportion of silent synapses compared to the vehicle-treated controls which in turn had baseline number of silent synapses (Figure 3.4F circles; saline WD1 grey, $24.13 \% \pm 3.83 \%, \mathrm{n} / \mathrm{m}=12 / 46$; cocaine + vehicle WD 20-30, $25.02 \pm 14.62, \mathrm{n} / \mathrm{m}=5 / 10$; cocaine + SYN119 WD 20-30, 50.10 $\pm 2.75, \mathrm{n} / \mathrm{m}=11 / 26$; one-way ANOVA $\mathrm{F}_{2,25}=7.835, \mathrm{p}<0.01$ ). Since removal of CP-AMPARs by SYN119 led to an increase in the number of NAcSh silent synapses (saline WD1 vs. cocaine + SYN119 WD 20-30, post hoc Bonferroni test $p<0.01$; cocaine + vehicle WD 20-30 
vs. cocaine + SYN119 WD 20-30, post hoc Bonferroni test $p<0.05$ ), it can be inferred that CPAMPARs that accumulate during prolonged withdrawal from cocaine-CPP do so, at least in part, in the cocaine-induced silent synapses. Therefore all the aforementioned results indicate that silent synapses which are induced in the NAcSh by cocaine exposure, mature by insertion of CP-AMPARs if a drug-related association (such as that in CPP) is learnt. It was yet to be tested whether such CP-AMPAR bearing synapses would be involved in long-term retention of drug-related memory (such as that measured by a CPP test post long-term withdrawal from the drug).

The CPP behavior results for the mice treated with SYN119 were no different from that of the vehicle-treated controls as all the mice remembered the association of the cocaine-paired chamber (Vehicle injection - PPS baseline to WD 1 to WD 20-30, $500.59 \pm 38.19$ to $751.52 \pm$ $17.81 \pm 678.06 \pm 43.86, n=8$, paired t-test baseline vs. WD $1 p<0.001$, paired t-test baseline vs. WD 20-30 p<0.01; SYN119 injection - PPS baseline to WD 1 to WD 20-30 from $517.58 \pm$ 40.64 to $764.40 \pm 26.86$ to $693.75 \pm 46.75, n=12$, paired t-test baseline vs. WD1 $p<0.001$, paired t-test baseline vs. WD 20-30 $p<0.01$ ). Thus in spite of having a high number of immature silent synapses in the NAcSh, the SYN119 treated animals could still retain memory of the cocaine-associated chamber. Therefore this data suggests that though silent synapses that are induced in adult animals upon cocaine exposure, mature by insertion of CP-AMPARs, this maturation may NOT be required to retain the long-term memory of drugassociated cues. 


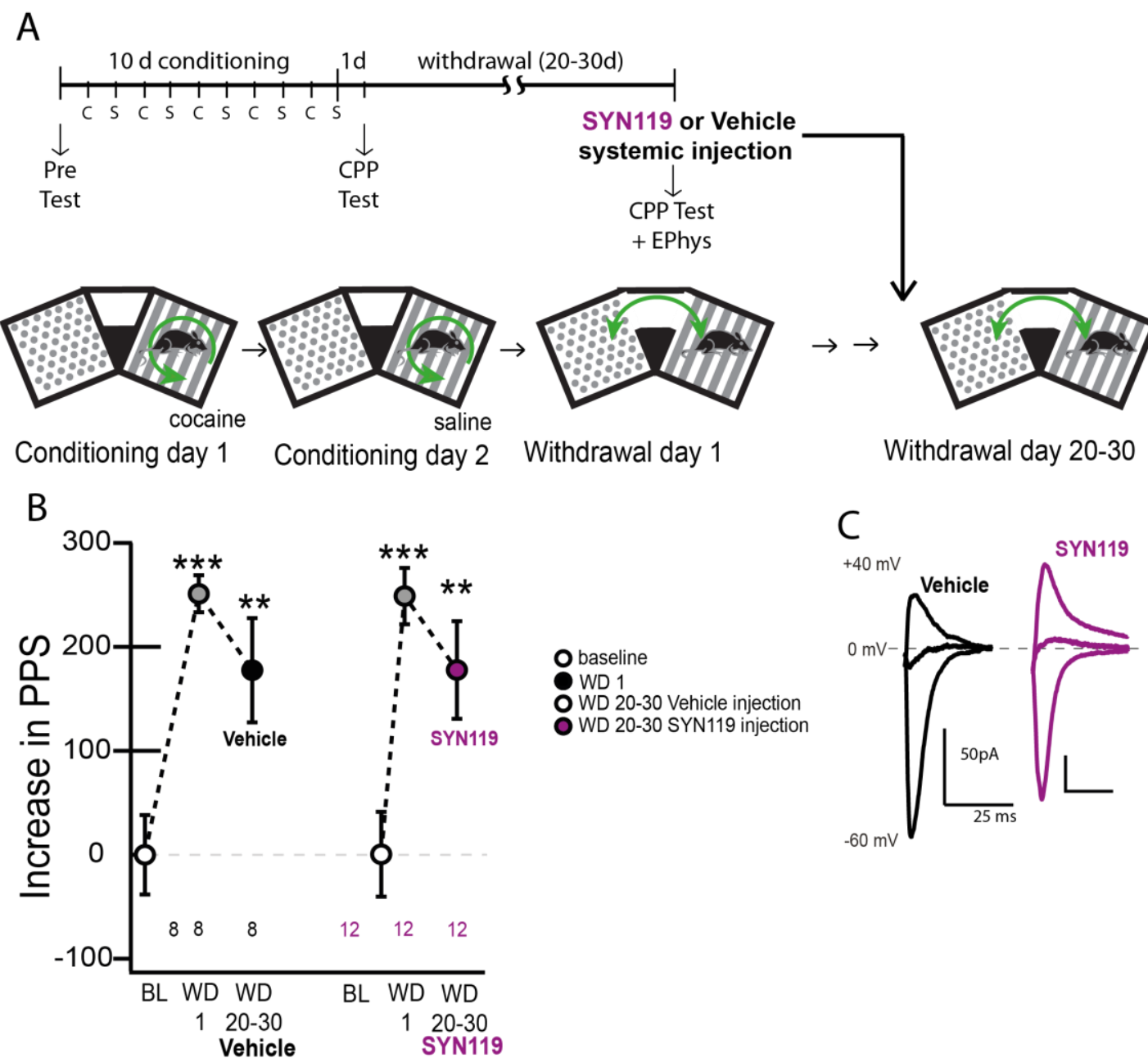

$\mathrm{D}$

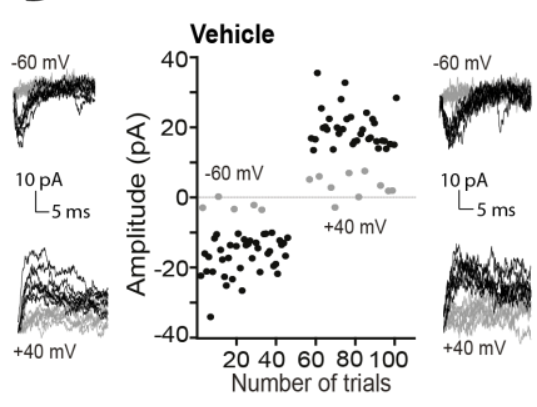

$\mathrm{E}$

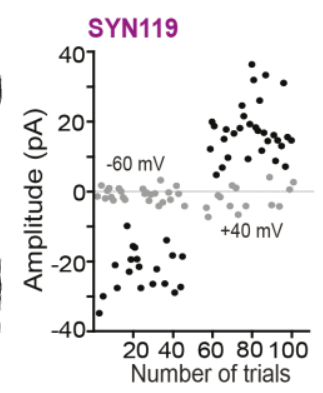

$\mathrm{F}$

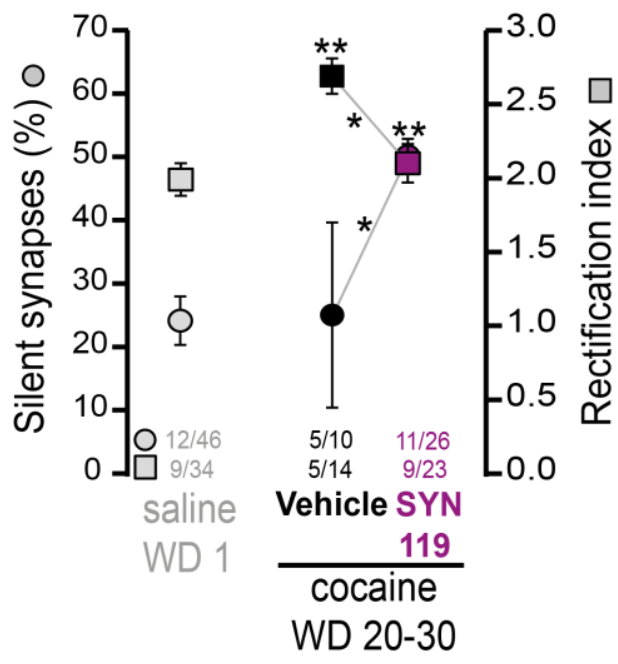

Figure 3.4 Positive allosteric modulation of mGluR1 through systemic SYN119 injection resilences the NAc synapses after long-term withdrawal from cocaine by removal of CP-AMPARs. Timeline and schematic diagram of the cocaine-CPP protocol followed by SYN119 injection $1 \mathrm{hr}$ prior to the behavior test and electrophysiology recordings immediately thereafter. (B) Wild type mice learn 
and remember the preference of cocaine-paired chamber upon SYN119 (or vehicle) treatment. The increase in the place preference score (PPS) is indicated by normalizing to the baseline score before commencement of the training. Number of animals used $(n)$ is indicated below each group. Statistical significance was assessed using paired t-test. (C) Example AMPA EPSCs evoked at $-60 \mathrm{mV}, 0 \mathrm{mV}$ and $+40 \mathrm{mV}$ during rectification index assay. (D) and (E) - Examples recordings of EPSCs evoked during minimum stimulation at 20-30 days of withdrawal from cocaine-CPP1, following vehicle injection (D) and SYN119 injection (E). (F) SYN119 treatment significantly reduces the rectification index at WD 2030 to baseline levels. Proportion of silent synapses increased significantly upon SYN119 treatment. $\mathrm{n} / \mathrm{m}$ is indicated in the figure below the respective data points. The saline control at 1 day of withdrawal from saline-CPP (same as shown in Figure3.1C) has been used an interleaved control.

\subsection{PSD-95 KO mice learn the cocaine-CPP association but do NOT retain it after prolonged withdrawal. The NAcSh has a high proportion of silent synapses that do NOT mature to CP-AMPAR containing synapses}

The signaling scaffold protein PSD-95, has been implicated to play a role in the maturation of silent synapses during brain development (Béïque et al., 2006; Huang et al., 2015a). Therefore to test whether it is also involved in the maturation of silent synapses that are induced in adulthood upon cocaine exposure, we used PSD-95 KO animals for the CPP behavior and electrophysiology from NAcSh MSNs as described above. The data from PSD$95 \mathrm{KO}$ animals was largely produced by a former post doc in my laboratory, Dr. Anna Suska. It is necessary to recapitulate these results in this thesis in order to understand the rationale behind the experiments that are yet to be described.

PSD-95 KO mice were used for the CPP behavior assay, wherein, they were treated with saline-only to check their baseline preference. With saline-CPP, PSD-95 KO mice do not develop a significant preference for any chamber (figure 3.5A, PPS baseline to WD 1: 507.63 \pm 32.92 to $546.38 \pm 80.13 ; n=8$, paired t-test $p=0.633$ ). However with cocaine-CPP the mice learn the association of the cocaine-paired chamber very well, as seen on WD1 but after 20 days of withdrawal from cocaine-CPP, the mice no longer remember this association (figure 3.5A, PPS baseline to WD 1 to WD 20: 518.91 $\pm 45.30, n=11$, to $809.94 \pm 44.76, n=$ 11 , to $530.72 \pm 51.22, n=10$; baseline vs. WD1, paired t-test $p<0.01$; baseline vs. WD 20 , paired t-test, $p=0.855)$. Thus the association learnt during the cocaine-CPP training is only transient in PSD-95 KO mice unlike the WT mice where this memory is retained for much longer. To check for cellular effects of loss of PSD-95, electrophysiology was performed on the NAcSh MSNs (Figure 3.5 B, C and D). It was observed that PSD-95 KO animals have a high number of baseline silent synapses in the NAcSh as observed on 1 day of withdrawal from saline-CPP. Due to cocaine exposure, the number of silent synapses becomes even 
higher as measured on WD 1 from cocaine-CPP. However, quite unlike the WT animals, silent synapses remain very high in PSD-95 KO mice even upon 20-25 days of withdrawal (figure 3.5E circles, saline WD1, 55.58\% $\pm 4.56 \%, \mathrm{n} / \mathrm{m}=6 / 21$; cocaine WD1, $74.23 \% \pm$ $1.91 \%, n / m=4 / 14$; cocaine WD20, $71.55 \% \pm 4.11 \%, n / m=5 / 20$; one way ANOVA $F_{2,12}=$ $6.414, p<0.05$; saline vs. cocaine WD 1 post hoc Bonferroni test $p<0.05$; saline vs. cocaine WD 20 post hoc Bonferroni test $p<0.05)$. Also, after prolonged withdrawal from cocaine-CPP, PSD-95 KO mice did NOT have a higher rectification index compared to baseline (figure 3.5E squares, saline WD 1, $1.75 \pm 0.09, \mathrm{n} / \mathrm{m}=4 / 24$; cocaine WD 1, $1.98 \pm 0.36, \mathrm{n} / \mathrm{m}=4 / 15$; cocaine WD $201.93 \pm 0.20, \mathrm{n} / \mathrm{m}=4 / 14$; one-way ANOVA $\left.F_{2,9}=0.2445, p=0.7881\right)$. Therefore in the PSD-95 KO mice, the NAcSh silent synapses do NOT mature to CP-AMPAR containing synapses. This result suggested that CP-AMPARs that accumulate in the NAcSh silent synapses, might be important for the long-term retention of drug-associated memory because in their absence, PSD-95 KO mice do not retain the memory of the association they had learnt.

A

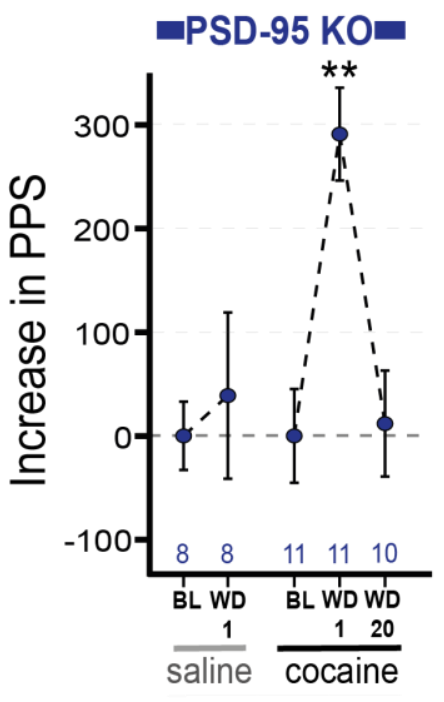

B

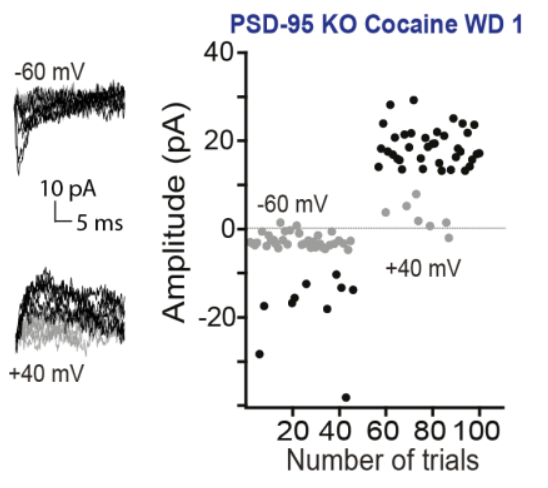

C

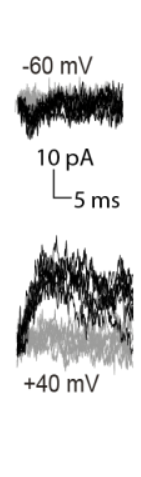

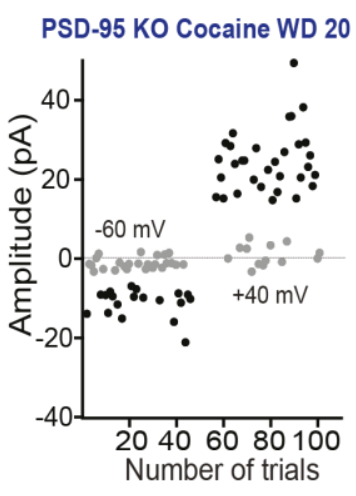
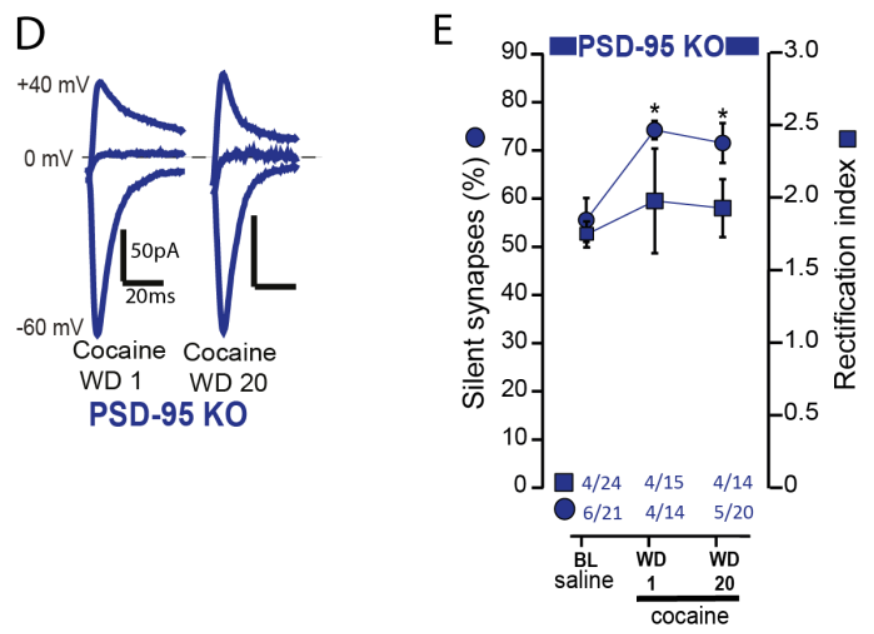

Figure 3.5 PSD-95 KO mice learn well the association of the cocaine-paired chamber in the CPP paradigm but not retain this memory after 20 days. They have a high baseline number of silent 
synapses in the NAcSh which increases upon cocaine-CPP but is NOT converted to rectifying CP-AMPAR-containing synapses upon long-term withdrawal from cocaine.

(A) The increase in the place preference score (PPS) is indicated by normalizing to the baseline score before commencement of the training. PSD-95 KO mice also do not develop a significant preference after saline-CPP, however following cocaine-CPP they develop a very high preference which falls back to baseline after 20-25 days of withdrawal. Number of animals used (n) is indicated below each group. Statistical significance was assessed using paired t-test. (B) and (C) EPSCs evoked at $-60 \mathrm{mV}$ and $+40 \mathrm{mV}$ by minimum stimulation (left side) over 100 trails from an example recording from PSD-95 KO mice at withdrawal day 1 (B) and withdrawal day 20-25 (C). (D) Example AMPA EPSCs evoked at $60 \mathrm{mV}, 0 \mathrm{mV}$ and $+40 \mathrm{mV}$ during rectification index assay in cells of the NAc of PSD-95 KO mice. (E) PSD-95 KO mice have a high baseline number of silent synapses (blue circles) which gets higher still upon cocaine-CPP and does not decrease during 20-25 days withdrawal from cocaine. Rectification index (blue squares) does not increase in PSD-95 KO mice after 20-25 days of withdrawal from cocaine-CPP. $\mathrm{n} / \mathrm{m}$ is indicated in the figure below the respective data points. These experiments were done in collaboration with A.Suska (see section 5 appendix, for details).

\subsection{SAP102 KO mice learn and remember the cocaine-CPP association even though the NAcSh has a high number of silent synapses lacking CP-AMPARs}

The SAP102 and PSD-95 are both proteins that belong to the DLG-MAGUK family and share similar domain structure. SAP 102 protein levels are known to be upregulated in the PSD-95 KO condition, which is indicative of a compensatory functional role (Bonnet et al., 2013; Cuthbert et al., 2007; Elias et al., 2006). Therefore I tested if SAP102 KO mice show the same phenotype as the PSD-95 KO mice with the same behavioral and electrophysiological assays.

SAP $102 \mathrm{KO}$ mice did not develop any preference when saline-CPP was performed with them (figure 3.6A, PPS baseline to WD 1: $523.34 \pm 84.55$ to $487.58 \pm 58.69, n=6$, paired test $\mathrm{p}=0.760$ ). However unlike PSD-95 KO, SAP102 KO mice learnt and also remembered the association of the cocaine-paired chamber in the CPP assay at least up till 20-days of withdrawal from cocaine (figure 3.6A, PPS baseline to WD 1 to WD 20: $494.60 \pm 25.48$ to $733.61 \pm 28.52$ to $662.35 \pm 38.02 ; n=19$; baseline vs. WD 1 paired t-test $p<0.001$; baseline vs. WD 20 paired t-test $\mathrm{p}<0.01$ ). To see if the difference in behavior of PSD-95 KO and SAP102 KO lies at the NAcSh cellular level, minimum stimulation protocol was done on NAcSh MSNs of SAP102 KO mice (figure 3.6B, C). The NAcSh silent synapse number also increases in SAP102 KO mice upon cocaine-CPP and it does not decrease during withdrawal from cocaine (Figure 3.6E circles, saline WD 1,32.71\% $\pm 4.84, \mathrm{n} / \mathrm{m}=6 / 14$; cocaine WD 1 $56.30 \% \pm 6.53, \mathrm{n} / \mathrm{m}=4 / 13$; cocaine WD $2056.55 \% \pm 5.01, \mathrm{n} / \mathrm{m}=8 / 16$; one way ANOVA $\mathrm{F}_{2,15}$ $=6.469, p<0.01$; saline vs. cocaine WD 1 post hoc Bonferroni test $p<0.05$, saline vs. cocaine WD 20 post hoc Bonferroni test $\mathrm{p}<0.01)$. Furthermore, NAcSh synapses of SAP102 KO mice 
also do not accumulate CP-AMPARs during withdrawal from cocaine-CPP as the rectification index (figure 3.6D) remains low at baseline levels at WD 20 (figure 3.6E, squares, saline WD $1,2.09 \pm 0.23, \mathrm{n} / \mathrm{m}=7 / 20$; cocaine WD 1, $2.08 \pm 0.10, \mathrm{n} / \mathrm{m}=4 / 14$; cocaine WD $201.87 \pm$ $0.10, \mathrm{n} / \mathrm{m}=8 / 31$; one-way ANOVA $\left.\mathrm{F}_{2,16}=0.6009, \mathrm{p}=0.5602\right)$. Therefore even though the cellular phenotype of PSD-95 KO and SAP102 KO mice is similar, their behavioral phenotype in the CPP assay was found to be the different.

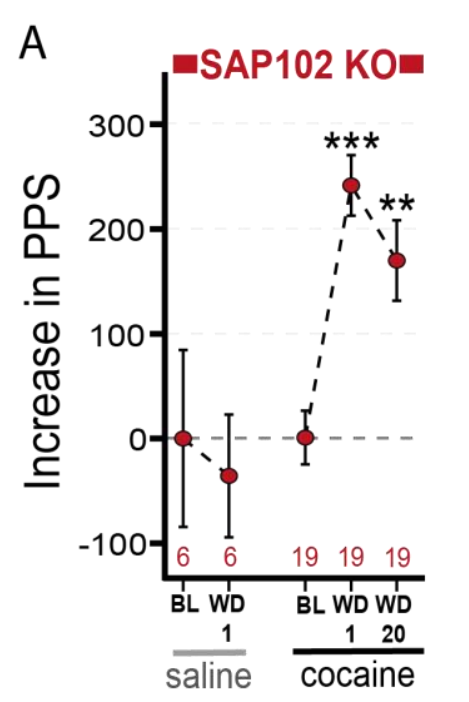

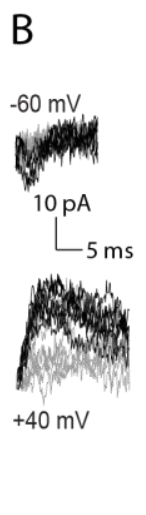

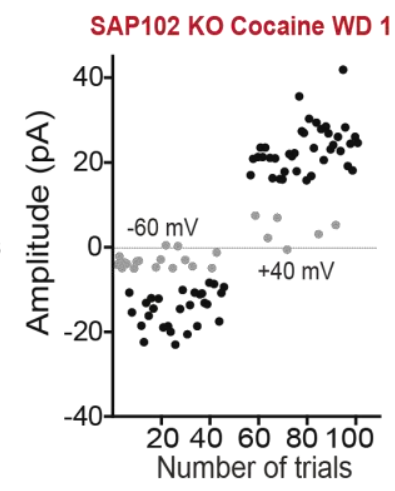

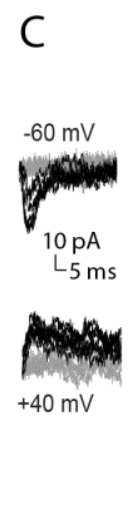

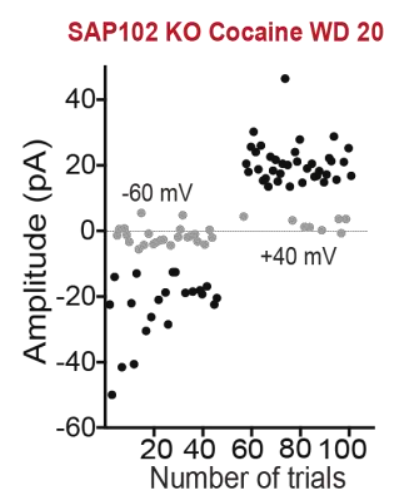

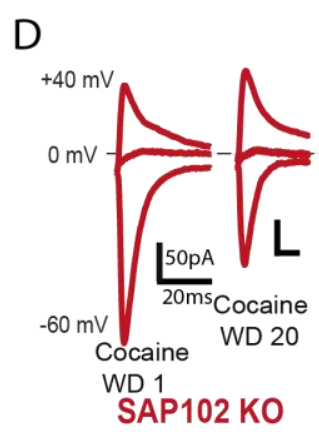

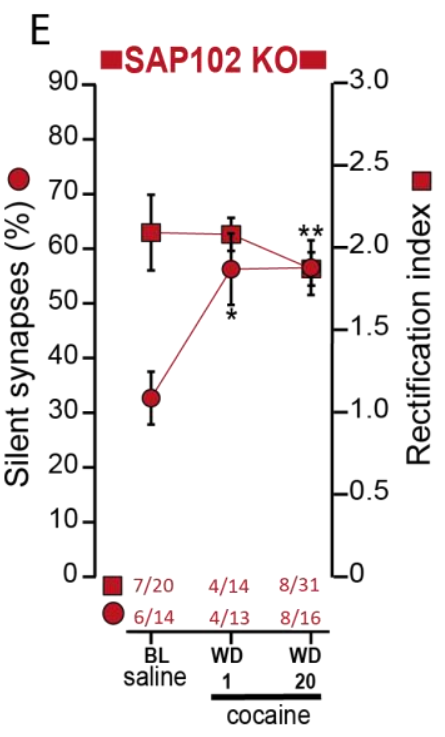

Figure 3.6 SAP102 KO mice learn and remember the association of the cocaine-paired chamber in the CPP paradigm. Silent synapses in these mice increase in number after cocaine-CPP but are NOT converted to rectifying CP-AMPAR-containing synapses upon long-term withdrawal from cocaine.

(A) The increase in the place preference score (PPS) is indicated by normalizing to the baseline score before commencement of the training. SAP102 KO mice do not develop a preference post saline-CPP but learn and remember the association after cocaine-CPP. Number of animals used (n) is indicated below each group. Statistical significance was assessed using paired t-test. (B) and (C) EPSCs evoked at $-60 \mathrm{mV}$ and $+40 \mathrm{mV}$ by minimum stimulation (left side) over 100 trails from an example recording from SAP102 KO mice at withdrawal day 1 (B) and withdrawal day 20-25 (C). (D) Example AMPA EPSCs evoked at $-60 \mathrm{mV}, 0 \mathrm{mV}$ and $+40 \mathrm{mV}$ during rectification index assay in cells of the NAc of SAP102 KO mice. (E) SAP102 KO mice have slightly higher number of baseline silent synapses which become higher upon cocaine exposure and do not decrease upon long-term withdrawal from cocaine. Rectification index does not increase in SAP102 KO mice after 20-25 days of withdrawal from cocaine- 
CPP. $\mathrm{n} / \mathrm{m}$ is indicated in the figure below the respective data points. These experiments were done in collaboration with A.Suska (see section 5 appendix, for details).

In figure 3.7 the CPP-behavior $(A)$ and electrophysiology (B) results of PSD-95 KO animals (in blue) and SAP102 KO animals (in red), described in section 3.4 and 3.5 respectively have been shown together with the corresponding results from WT animals (in grey, shown before in figure 3.1 and figure 3.2) for the sake of comparison. In context of behavior, WT animals and SAP102 KO animals exhibit the same pattern of learning the association of the cocaine-paired chamber during CPP and remembering it up to 20-25 days of withdrawal. PSD-95 KO mice on the other hand have a strikingly different behavioral phenotype. They seem to learn the CPP-association even better than WT animals as seen by the increase in PPS on WD 1, however they forget this association completely by WD 20-25.

In WT mice, silent synapses are higher after cocaine-CPP at WD1 but fall back to baseline by WD 20 (figure 3.7B grey circles, saline WD 1, 24.13\% $\pm 3.83 \%$; cocaine WD $146.90 \% \pm$ 3.015, cocaine WD 20 19.61\% 4 4.87\%; one-way ANOVA $F_{2,29}=15.9, p<0.0001$; saline WD 1 vs. cocaine WD 1 post hoc Bonferroni test $p<0.001)$. Rectification index was shown to increase after cocaine-CPP in WT mice after 20-25 day long withdrawal from cocaine (figure 3.7B grey squares, saline WD 1, $1.99 \pm 0.11$; cocaine WD 1, $1.96 \pm 0.08$; cocaine WD 20, $2.45 \pm 0.12$; one way ANOVA $F_{2,25}=5.761, p<0.01$; saline WD 1 vs. cocaine WD 20, post hoc Bonferroni test $\mathrm{p}<0.05)$. It is noteworthy that PSD-95 KO mice have the highest number of baseline silent synapses (figure 3.7B, circles; WT saline WD1 vs. PSD-95 KO saline WD1, ttest $p<0.001$; PSD-95 KO saline WD1 vs. SAP102 KO saline WD1, t-test, $p<0.01$ ). Also, both PSD-95 KO and SAP102 KO mice show different phenotype than WT mice on the cellular level of NAcSh. Cocaine exposure induces more silent synapses in the NAcSh of both PSD$95 \mathrm{KO}$ and SAP102 KO mice, which do not mature during prolonged withdrawal from cocaine-CPP. Therefore the lack of either MAGUK protein, PSD-95 or SAP102 prevents the maturation of silent synapses into CP-AMPAR containing synapses, which happens by default in WT animals during withdrawal from cocaine-CPP. However this process is not required for maintaining the long-term memory of drug-associated cues, as shown by the SAP102 KO. PSD-95 is known to play a crucial role in AMPAR recruitment to synapses via its interaction with Stargazin (Schnell et al., 2002) while SAP102 is known to compensate for the loss of PSD-95. Therefore an optimal level of MAGUK proteins might be necessary for proper stabilization of AMPARs at the NAcSh synapses. However if the MAGUK proteins are absent since birth, in the case of the $\mathrm{KO}$ animals, alternative or compensatory mechanisms could 
have been put in place during brain development to maintain the retention of long-term memory.

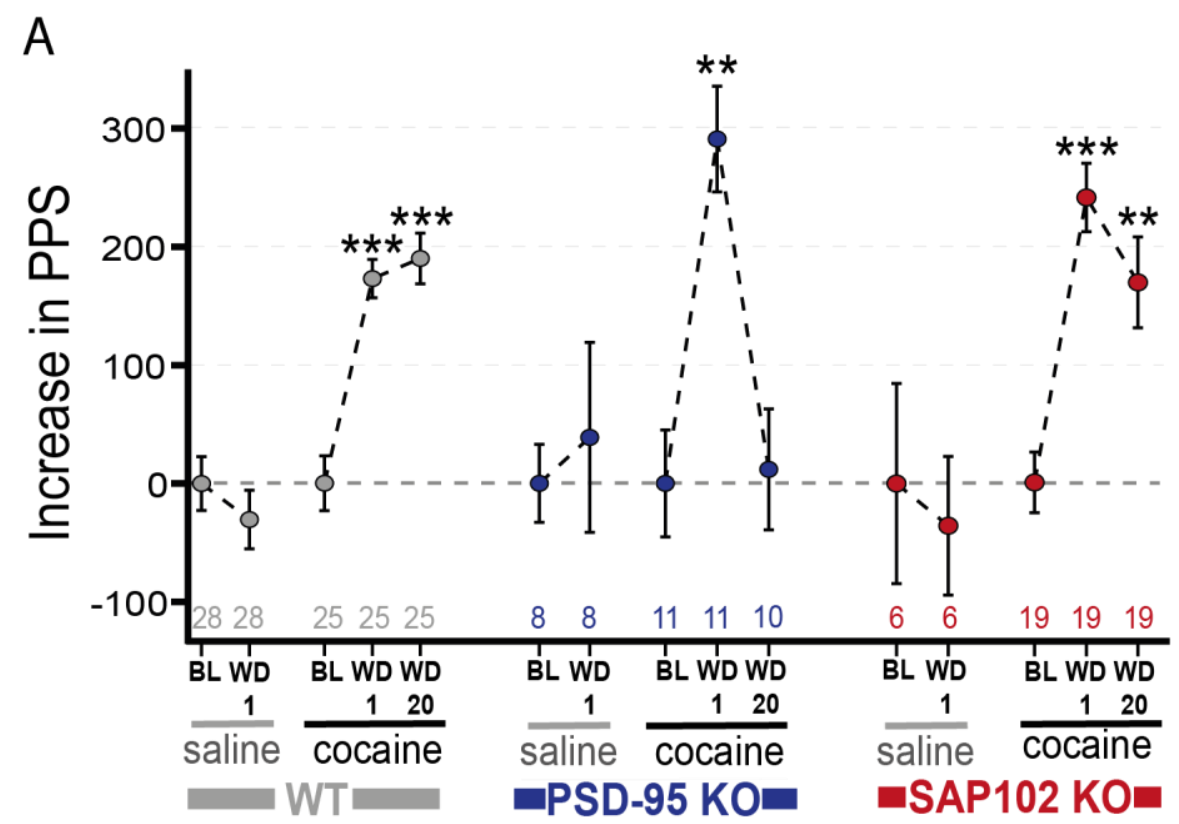

B

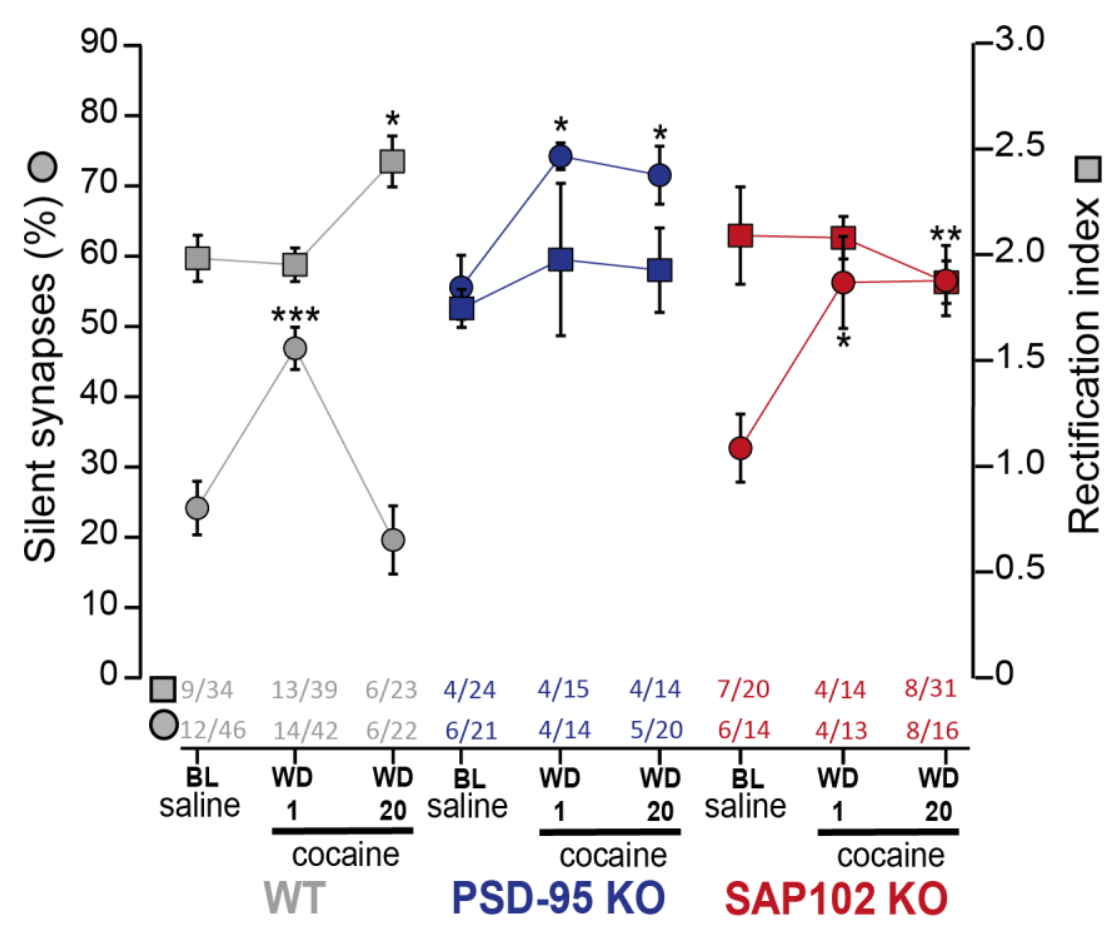

Figure 3.7 Summarized results of CPP behavior assay (A) and electrophysiological assays (B) performed on NAcSh cells of WT, PSD-95KO and SAP102 KO mice.

Data of the WT mice (grey), was shown before in Figure 3.1 and is represented here again for comparison. Data from PSD-95 KO animals (blue) and SAP102 KO animals (red) is same as that shown in Figure3.5 and 3.6 respectively. 


\subsection{NAcSh-restricted knock-down of PSD-95 does not impair retention of drug-associated memory in WT or SAP102 KO mice}

In order to dissect the role of PSD-95 in maturation of cocaine-induced silent synapses specifically in the NAcSh, I decided to knock-it down locally in the NAcSh. I used a shRNA against PSD-95 named 'sh95' along with a GFP marker and packaged it into AAV8 particles (for details please refer to materials and methods section 2.5). The AAV8 viral solution was used for stereotaxic injections into the NAcSh of mice, in order to have a NAcSh-restricted RNAi-mediated knockdown of PSD-95. After 2-3 weeks, once the mice totally recovered from the stereotaxic surgery and enough time had been given for the acute reduction of PSD-95 protein levels, I proceeded to do the CPP behavior assay on these animals as usual (figure 3.8A). The mice were used for electrophysiology either after the first CPP test at WD 1 or after they underwent 20-25 days of undisturbed withdrawal in their home cages, followed by the last CPP test. The accurate locations of the stereotaxic viral injection and the associated spread of the virus infection were estimated by checking the GFP fluorescence in the NAcSh region (figure $3.8 \mathrm{~B}$ left). The approximate infection rate was assessed either by confocal microscopy or during electrophysiology with high magnification objectives of the microscope. The infection rate was approximately $90-95 \%$ in an area well covered under the spread of the virus injection, as seen by the co-localization of GFP positive NAcSh MSNs and DAPI-stained nuclei (figure 3.8B right). Therefore these animals could be used for a behavioral assay as subjects lacking PSD-95 in the NAcSh. As an injection control, I injected a virus carrying the GFP marker or a luciferase shRNA along with GFP in the same AAV8 vector. All the control group animals were treated in a manner identical to that of sh95 injected animals.

The stereotaxic injection procedure or the AAV8 infection into the NAcSh did not cause any impairment in the CPP behavioral assay. WT animals injected with control virus learnt and remembered the cocaine-paired association of CPP till 20-25 days of withdrawal from cocaine-CPP (figure 3.8C grey, PPS baseline to WD 1 to WD 20: $523.05 \pm 20.79, n=12$, to $687.12 \pm 21.04, n=12$, to $616.14 \pm 30.18, n=8$; baseline vs. WD 1 paired t-test $p<0.001$; baseline vs. WD 20 paired t-test $p<0.05)$. The WT animals injected with sh95 also remembered the association they learnt during the CPP training (figure 3.8C blue, PPS baseline to WD 1 to WD 20: $529.22 \pm 17.00, n=26$, to $691.08 \pm 19.45, n=26$, to $721.24 \pm$ 29.24, $n=26$; baseline vs. WD 1, paired t-test $p<0.001$; baseline vs. WD 20, paired t-test 
$p<0.01)$. Therefore the behavioral phenotype of NAc-restricted PSD-95 knock-down was different than that of the PSD-95 KO.

There may have been a possibility that functional compensation by SAP102 at the cellular scale of NAcSh MSNs might be responsible for the lack of a behavioral effect of NAcrestricted PSD-95 knock-down. Therefore I used SAP102 KO mice for the NAc-restricted PSD-95 knock-down using the same RNAi approach. This was done in order to mimic a PSD95/SAP102 double knockout-like condition at the NAcSh. SAP102 KO mice when injected with the control virus did not show any impairment in learning the CPP association or in its long-term retention (figure 3.8 red, PPS baseline to WD 1 to WD 20: $538.23 \pm 43.92$ to $795.33 \pm 24.28$ to $882.41 \pm 29.16, n=8$; baseline vs. WD $1, p<0.001$; baseline vs. WD 20, $\mathrm{p}<0.01)$. Furthermore, SAP102 KO mice injected with sh95 in the NAc also learnt and remembered the association of the CPP assay (figure 3.8 green, PPS baseline to WD 1 to WD 20: $527.85 \pm 25.12, n=19$, to $706.25 \pm 21.80, n=19$, to $690.86 \pm 46.70, n=10$; baseline vs. WD 1, paired t-test $p<0.001$; baseline vs. WD 20, paired t-test $p<0.01$ ). Therefore the MAGUKs, PSD-95 and SAP 102 in the NAcSh, are NOT required for the overall retention of drug-associated memory in the animal. 
A

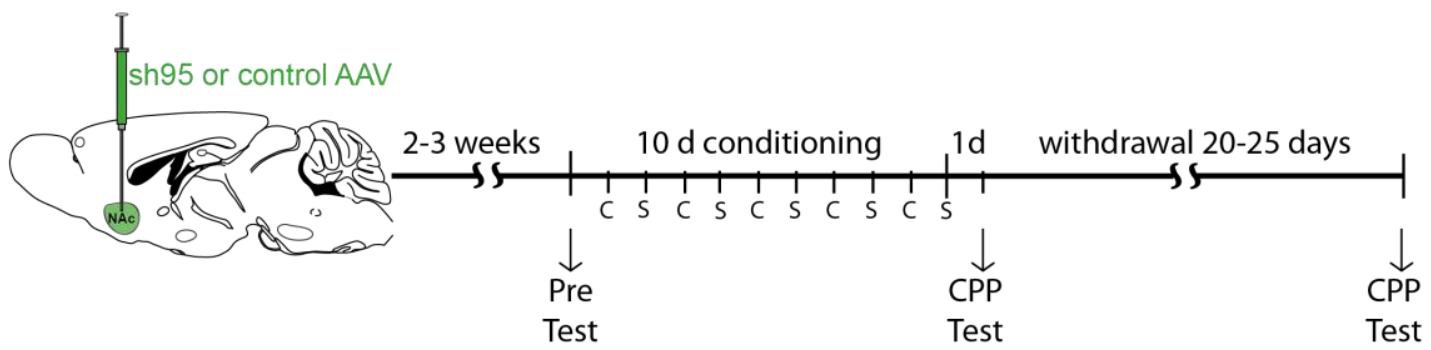

B

DIC

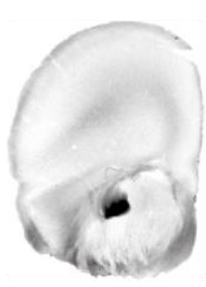

GFP

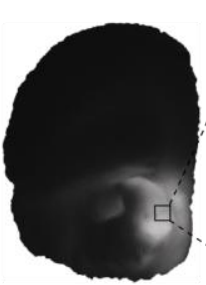

DAPI

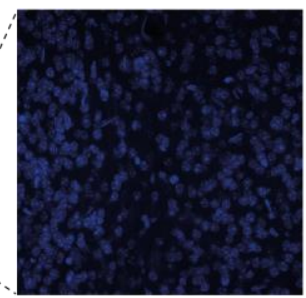

GFP

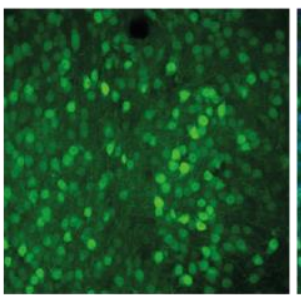

merge



C

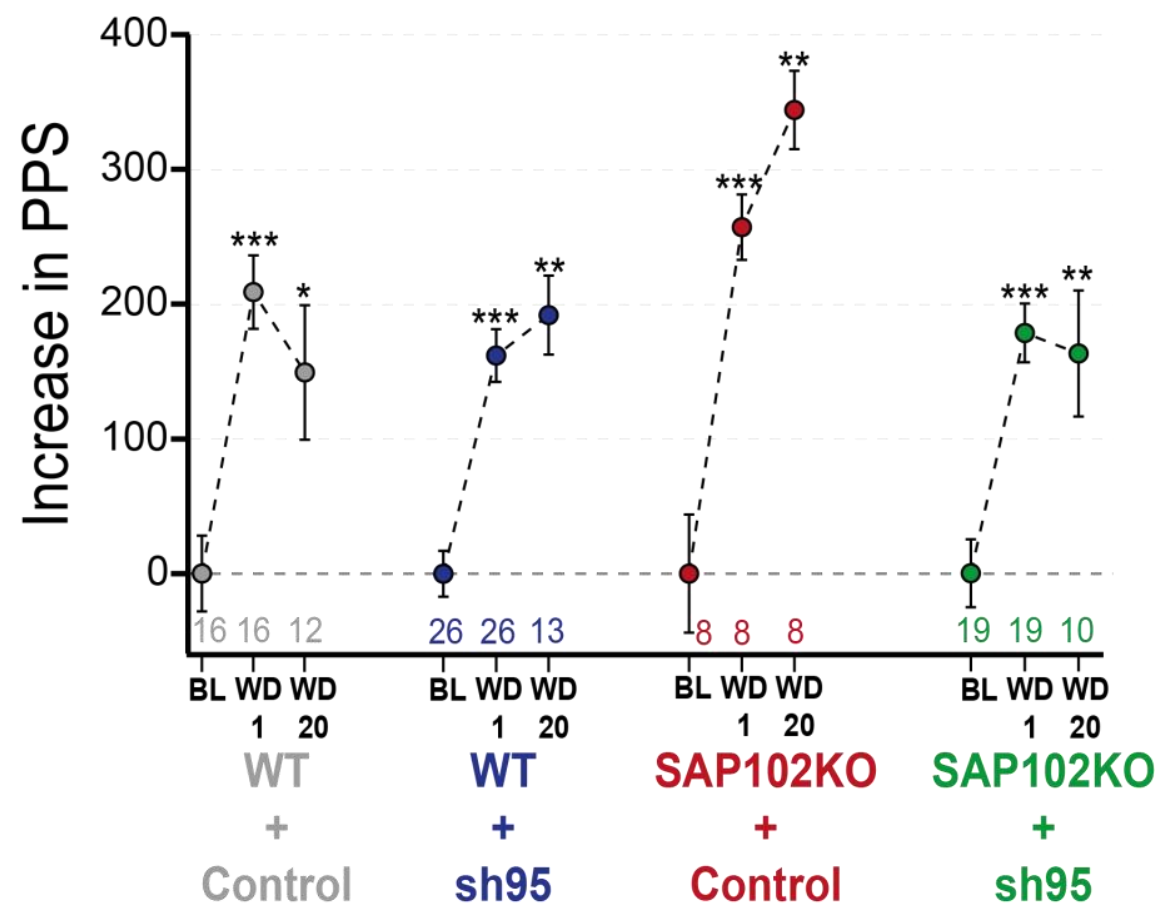

Figure 3.8 NAcSh-restricted PSD-95 knock down does not impair long-term retention of CPP memory in wild type mice and SAP102KO mice.

(A) Timeline of the stereotaxic injection of AAV8 virus in the NAcSh followed by the CPP protocol and 20-25 days of withdrawal from cocaine-CPP. (B) Light (DIC) and fluorescent (GFP) images showing the expression site of the AAV8 virus in the NAcSh followed by confocal images of a selected area of the NAcSh showing infected cells (GFP) among total number of cells (DAPI stained nuclei).Scale bar, 
$50 \mu \mathrm{m}$. (C) The increase in the place preference score (PPS) is indicated by normalizing to the baseline score before commencement of the training. The stereotaxic injection of AAV8 virus into the NAcSh, in wild type mice (grey) did not impair the CPP behavioral task or the associated memory retention. Stereotaxic injection of sh95 containing AAV8 into WT mice (blue) had no effect on the retention of CPP memory. Stereotaxic injections of AAV8 into the NAcSh of SAP102KO mice (red) did not impair the CPP behavior task. Creating a NAcSh-restricted double KO of SAP102 and PSD-95 by using SAP102KO animals for sh95 AAV8 injections in the NAc (green) did not impair long-term retention of CPP memory. Some of the experiments were done in collaboration with A.Suska (see section 5 appendix, for details).

\subsection{PSD-95 and/or SAP102 are required in the NAcSh MSNs for maturation of silent synapses}

The mice used for stereotaxic injections in section 3.6 were also used for electrophysiology from the NAcSh MSNs. This time the cells chosen for recordings were the ones that were clearly GFP positive, and therefore infected with virus (figure 3.9A). Rectification index assay was performed on GFP positive cells of sh95 injected mice at WD 1 and WD 20, as well as control virus injected mice at WD 20 (figure 3.9B). The stereotaxic injection procedure did not impair the accumulation of CP-AMPARs in the NAcSh during prolonged withdrawal from cocaine-CPP, as seen by the high rectification index at WD 20 in GFP positive cells of WT animals injected with control virus (figure 3.9G, grey square, $2.87 \pm$ $0.27, n / m=4 / 12)$. The rectification index of WT animals injected with sh95 into the NAcSh, did not increase upon withdrawal from cocaine-CPP and was lower than that of WT animals injected with control virus (figure 3.9G blue squares, WT+sh95 cocaine WD 1, $2.44 \pm 0.22$, $\mathrm{n} / \mathrm{m}=4 / 11 ; \mathrm{WT}+$ sh95 cocaine WD 20, $2.14 \pm 0.15, \mathrm{n} / \mathrm{m}=10 / 20 ;$ t-test $\mathrm{p}=0.292 ; \mathrm{WT}+$ sh95 cocaine WD 20 vs. WT+control cocaine WD 20, t-test, $p<0.05)$. Rectification index of SAP102 KO NAcSh cells infected with sh95 after 20 days of withdrawal from cocaine-CPP was not higher than at WD 1 or than of the control infected cell at WD 20 (figure 3.9G green squares, SAP102 KO+sh95 cocaine WD 1, $1.90 \pm 0.16, \mathrm{n} / \mathrm{m}=3 / 11 ;$ SAP102 KO+sh95 cocaine WD $20,2.10 \pm 0.06, n / m=4 / 14$; t-test $p=0.244$; red square SAP102 $\mathrm{KO}+$ control WD 20, $2.09 \pm$ 0.17, $\mathrm{n} / \mathrm{m}=3 / 9$; SAP102 KO+sh95 WD 20 vs. SAP102 $\mathrm{KO}+$ control WD 20, t-test $\mathrm{p}=0.949$ ). Therefore in cells lacking PSD-95 and/or SAP102, CP-AMPARs do not accumulate during withdrawal from cocaine-CPP.

The number of silent synapses in wild type NAcSh cells infected with sh95 was very high at the baseline as well as at WD 20 compared to cells infected with control virus (figure 3.9G blue circles, WT+sh95 cocaine WD1, $59.70 \% \pm 4.87 \%, \mathrm{n} / \mathrm{m}=5 / 13$; WT+sh95 cocaine WD $20,48.25 \% \pm 6.69 \%, n / m=8 / 20$; t-test $p=0.247$; grey circle, WT+control cocaine WD 20, 
$17.67 \% \pm 2.77 \%$; $W T+$ sh95 cocaine WD 20 vs. $W T+$ control cocaine WD 20 , t-test $p<0.05$ ). Also SAP102 KO cells infected with sh95 have a higher number of silent synapses at WD 1 and WD 20 (figure $3.9 \mathrm{G}$ green circles, SAP102 KO + sh95 cocaine WD 1, 71.18\% $\pm 3.71 \%$, $\mathrm{n} / \mathrm{m}=6 / 20$, SAP102 KO + sh95 cocaine WD 20, 76.46\% $\pm 1.77 \%, \mathrm{n} / \mathrm{m}=5 / 17$; t-test $\mathrm{p}=0.261$; red circle, SAP102 KO + control cocaine WD 20, 59.39\% $\pm 5.87 \%, \mathrm{n} / \mathrm{m}=3 / 8$; SAP102 KO + control cocaine WD 20 vs. SAP102 KO + sh95 cocaine WD 20, t-test $p<0.05)$. Therefore the collective loss of SAP102 and PSD-95 results in an even higher number of silent synapses in the NAcSh, compared to the individual loss of these proteins. These results indicate that MAGUKs are important for maturation of silent synapses and in their absence, NAcSh synapses remain largely immature. However these synapses of the NAcSh may not govern the overall drug-related behavior of the animal. This calls for a thorough examination of other brain areas to determine where the drug-associated long-term memory is stored. 


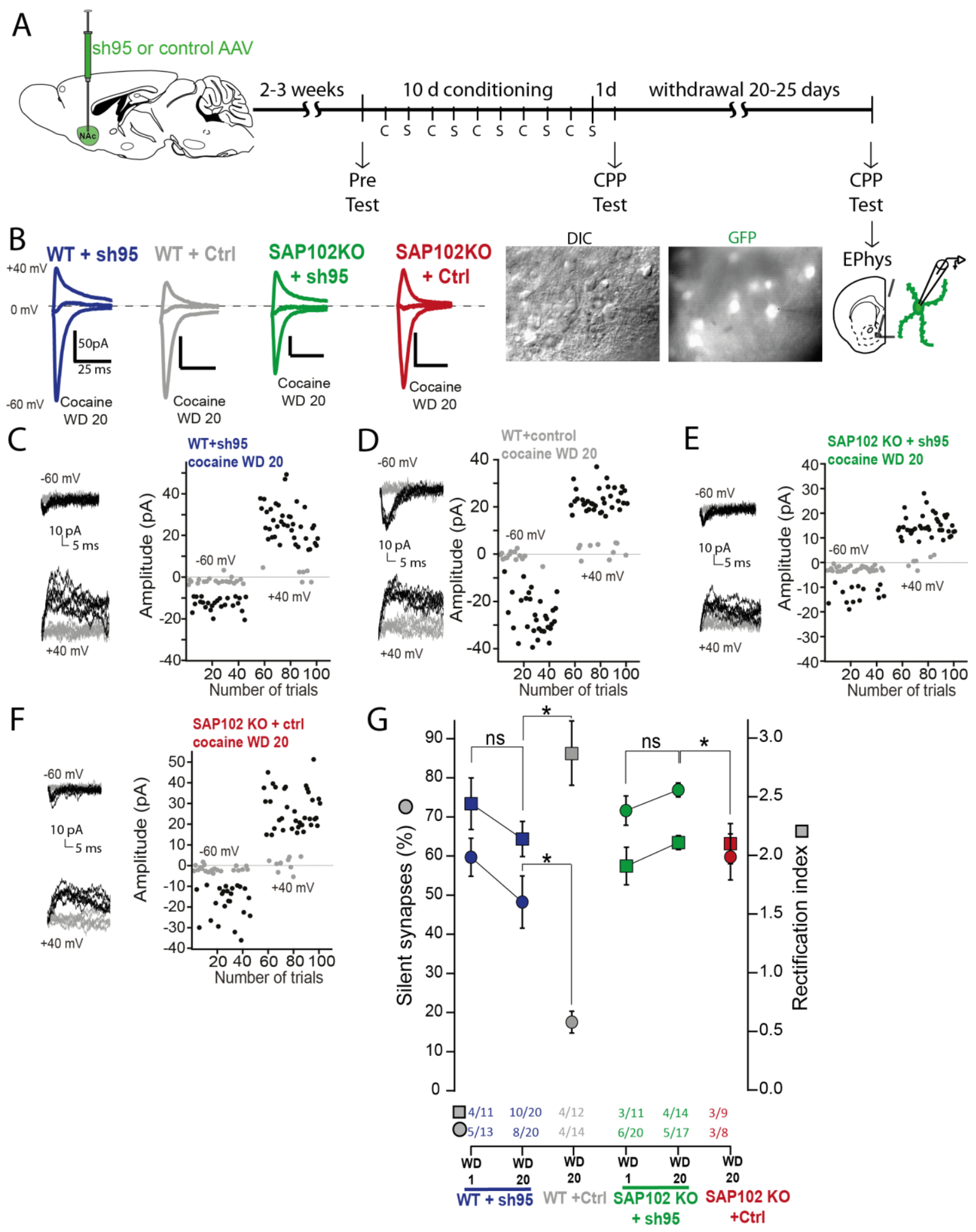

Figure 3.9 NAc restricted PSD-95 knock-down has a cellular phenotype in wild type mice and SAP102KO mice.

(A) Timeline of the stereotaxic injection of AAV8 virus in the NAcSh followed by the CPP protocol and 20-25 days of withdrawal from cocaine, wherein electrophysiology was done on GFP+ve cells. The 
DIC and GFP pictures show infected NAcSh MSNs (B) Example AMPA EPSCs evoked at $-60 \mathrm{mV}, 0 \mathrm{mV}$ and $+40 \mathrm{mV}$ during rectification index assay in GFP positive (AAV8 infected) cells of the NAc of the mice. (C), (D), (E) and (F) EPSCs evoked at $-60 \mathrm{mV}$ and $+40 \mathrm{mV}$ by minimum stimulation (left side) over 100 trails from an example recording from WT or SAP102 KO mice injected with sh95 or control virus at withdrawal day 20-25. (G) Summarized results of number of silent synapses and rectification index in GFP positive (AAV8 infected) cells of the NAc of mice. WT cells infected with sh95 virus animals have a high number of silent synapses post cocaine-CPP which remains high during withdrawal as compared to the WT cells injected with control virus. Rectification index is not high in WT cells infected with sh95 after long-term withdrawal from cocaine. In the NAc of SAP102 KO animals, cells infected with sh95 virus have a high number of silent synapses which remains high upon long-term withdrawal from cocaine-CPP, compared to the SAP102 KO animals injected with control virus. $\mathrm{n} / \mathrm{m}$ is indicated in the figure below the respective data points. Some of the experiments were done in collaboration with A.Suska (see section 5 appendix, for details).

\subsection{NAcSh-restricted knock down of PSD-95 in rats, does not affect cocaine self-administration}

The results described in section 3.6 and 3.7 specify that the maturation of cocaine-induced silent synapses to CP-AMPAR containing synapses in the NAcSh, during withdrawal from cocaine-CPP, is dependent on PSD-95 most likely through its role in stabilization of AMPAR at these synapses. The role of SAP102, being a compensatory MAGUK seems to be redundant for this process. However, this process was shown to be NOT essential for retaining the long-term drug-associated memory as tested in the CPP assay. CP-AMPARs have been shown to accumulate in NAc synapses during withdrawal from cocaine selfadministration (SA) and are known to mediate the time-dependent increase in cue-induced cocaine seeking termed as 'incubation of cocaine craving' (Conrad et al., 2008; McCutcheon et al., 2011a; Lee et al., 2013; Loweth et al., 2014a). Therefore it was nevertheless possible that CP-AMPARs which accumulate in the NAcSh synapses in the context of drug-related learning do not code for long-term memory but are only responsible for incubation of cocaine craving and thus the 'intensity' of a drug-related behavioral response. Therefore it was yet to be tested whether PSD-95 in NAcSh synapses, which has been shown to be essential for maturation of silent synapses to CP-AMPAR containing synapses during prolonged withdrawal from cocaine, would also be essential for developing incubation of craving. I therefore decided to test this using the cocaine-SA model, in collaboration with Prof. Yan Dong's laboratory at the University of Pittsburgh, PA, USA.

I used wild type rats and performed stereotaxic injections of sh95 AAV8 bearing a GFP marker, into the NAcSh in order to induce RNAi-mediated knock-down of PSD-95 (figure 3.10A). As an injection control, the same AAV8 vector was used with shRNA against luciferase. The locus of the injection and the spread of the virus infection was analyzed at the 
end of the entire experiment, by perfusing the rat brain with a fixative and imaging NAcSh slices. The spread of the virus as seen from the GFP fluorescence was in the correct region, but as the NAcSh is larger in the rat brain than in the mouse brain, the virus spread was not as much as in the mouse brain (figure 3.10B left). The infection rate of the virus was however similar to that observed in the mouse brain, as seen by the co-localization of GFP positive NAcSh MSNs and DAPI-stained nuclei (figure 3.10B right). Since not all the sh95 virus injections had infected the NAcSh tissue in an equally good way, I anticipated that the variance between individually injected rats might be very high. Therefore after checking the sh95 virus spread in the NAcSh of all rats post-hoc, I scored them on a scale of 0 to 5 and only the animals which scored above 2.5 been included in the data set.

In order to use these rats having NAcSh-restricted knockdown of PSD-95 for intravenous cocaine-SA, catheters were implanted via a surgery in the jugular vein. This was done either along with the stereotaxic surgery or 1 week after the rats recovered from it. Overall, the rats were given at least 1 week to recover from any surgical procedure and at least 2 weeks were given for sufficient viral infection in the NAcSh before the commencement of the cocaine-SA procedure (figure 3.10A). The rats were allowed to learn the SA procedure in an initial overnight training session wherein nose pokes into the active hole resulted in an intravenous (i.v) infusion of cocaine along with the concomitant cues (for details refer to materials and methods section 2.2.3). After a rest of one day, the rats were subjected to $2 \mathrm{hr}$ training sessions for 5 consecutive days, wherein number of active nose pokes that resulted in i.v infusion of cocaine were recorded. As SA control, some of the rats were trained with the vehicle saline and processed identically. It was seen that rats injected in the NAcSh with the control virus, got far more cocaine infusions during the $2 \mathrm{hr}$ training sessions than the ones used for saline-SA (figure 3.10C left; no. of cocaine infusions, black circles: Day $1-34.4 \pm 6.5$ , Day $2-27.67 \pm 4.2$, Day $3-30.9 \pm 4.5$, Day $4-34.0 \pm 5.7$, Day $5-30.6 \pm 5.3, n=9$; no of saline infusions, open circles: Day $1-3.2 \pm 1.1$, Day $2-2.7 \pm 1.2$, Day $3-2.7 \pm 0.9$, Day $4-$ $3.5 \pm 0.6$, Day $5-3.7 \pm 1.6, n=4)$. Therefore the stereotaxic injection did not impair the rats from learning the self-administration procedure as they learned to get more infusions of cocaine, since they were more rewarding than saline. The rats injected with sh95 virus also learnt the SA procedure well as they got more cocaine infusions than the rats which were trained with saline (figure 3.10C right; no. of cocaine infusions, green circles: Day $1-34.4 \pm$ 10.2, Day $2-20.3 \pm 3.1$, Day $3-17.9 \pm 3.2$, Day $4-17.9 \pm 4.3$, Day $5-20.6 \pm 4.4, \mathrm{n}=7$; no of saline infusions, open circles: Day $1-6.4 \pm 1.0$, Day $2-4.0 \pm 0.6$, Day $3-3.4 \pm 1.1$, Day $4-4.4 \pm 0.6$, Day $5-5.4 \pm 1.5, n=7$ ). Since all the rats learnt how to self-administer 
the cocaine and also found it rewarding, shown by the increased intake of cocaine over saline, these rats could be used for evaluating incubation of cocaine craving.

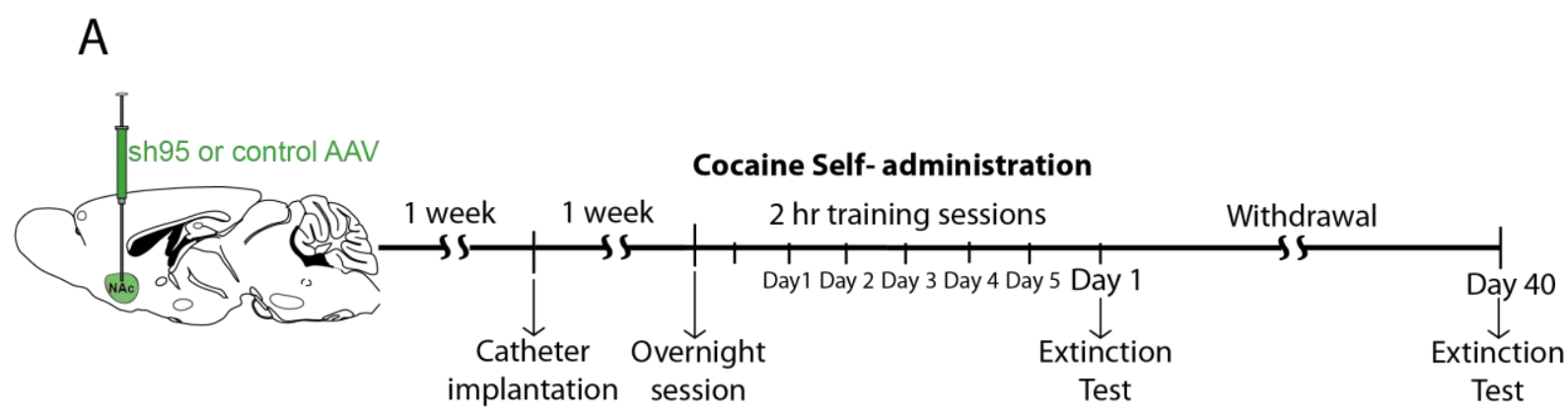

B
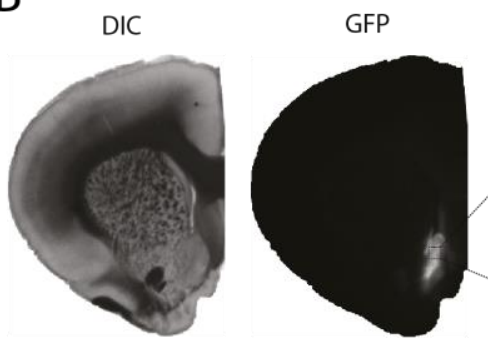

DAPI

GFP

merge
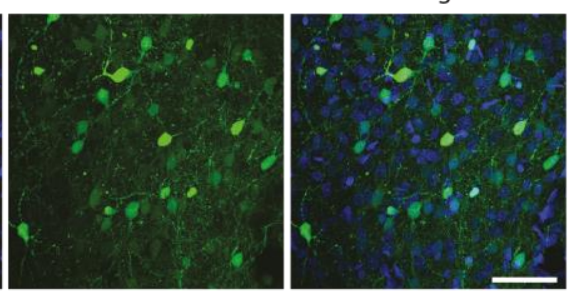

C

WT + control

WT + sh95

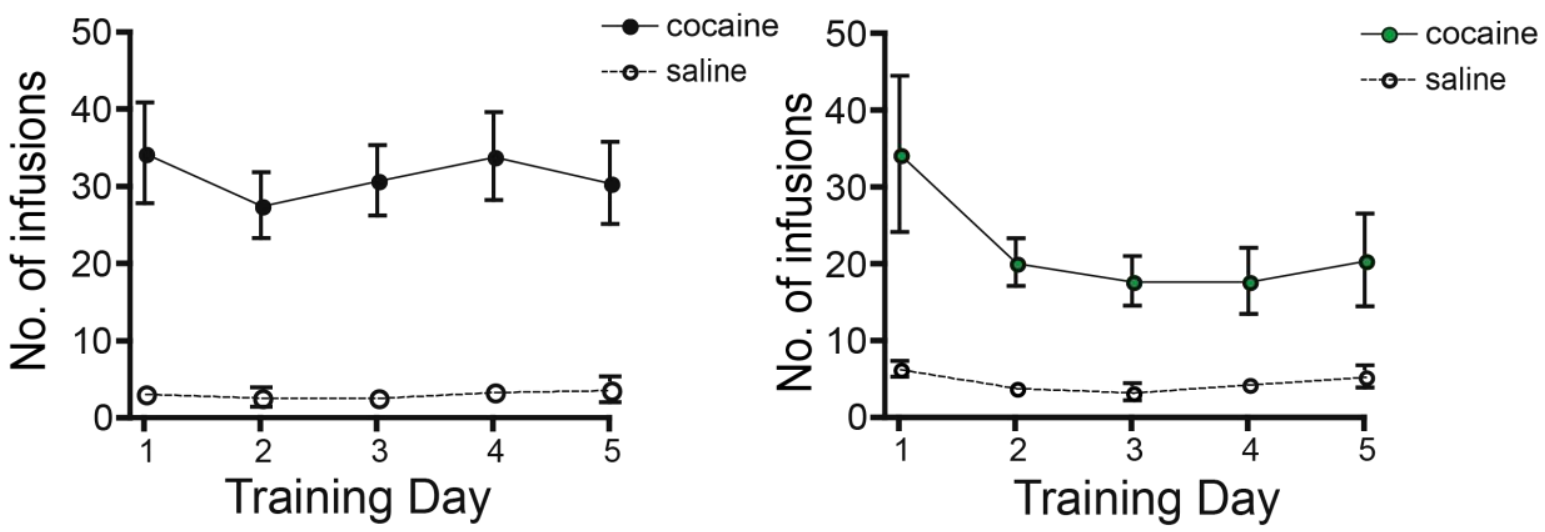

Figure 3.10 NAcSh-restricted knockdown of PSD-95 does not impair self-administration of cocaine in rats.

(A) Timeline of the stereotaxic injection of AAV8 virus into the rat NAcSh followed by the catheter implantation, cocaine self-administration training and withdrawal. (B) Light (DIC) and fluorescent (GFP) images showing the expression site of the AAV8 virus in the NAcSh followed by confocal images of a selected area of the NAcSh showing infected cells (GFP) among total number of cells (DAPI stained nuclei). Scale bar, $50 \mu \mathrm{m}$. (C) Summarized results of saline or cocaine self-administration training of rats injected with sh95 AAV8 (green) or control AAV8 stereotaxic injection in the NAcSh. 


\subsubsection{NAcSh-restricted knock down of PSD-95 in rats, does not impair incubation of cocaine craving}

Incubation of cocaine craving refers to the increase in cue-induced cocaine seeking behavior that occurs during withdrawal from cocaine-SA. Cue-induced cocaine seeking is performed by conducting extinction tests in the absence of the drug, wherein an active nose poke by the animal results in the concomitant cues but no cocaine infusion takes place. A significant increase in the number of active nose pokes in an extinction test performed after prolonged withdrawal from cocaine-SA, compared to that performed 1 day after the five training sessions indicates the presence of incubation of craving. It suggests that animals 'crave' more for the drug reward and is considered to be mediated by the CP-AMPARS accumulated in the NAc over the withdrawal period. Thus the rats used for SA were subjected to a $1 \mathrm{hr}$ extinction test on withdrawal day 1 wherein the number of active nose pokes was recorded to indicate the cue-induced cocaine-seeking. The number of inactive nose pokes was also recorded, to indicate the overall activity level of the rats, as hyperactivity increases the number of nose pokes. This was also recorded for the saline controls, although they got much lesser infusions during the training sessions (figure 3.10C). The rats were then left undisturbed in their home cages during withdrawal and subjected to an identical extinction test on WD 40. The rats injected with control virus in NAcSh exhibited significant incubation of craving as seen by the increase in number of active nose pokes from WD 1 to WD 40 (figure 3.11A left, number of active nose pokes, black circles: WD 1, 47.0 \pm 7.1 to WD 40, $101.0 \pm$ 14.7; $n=9$; paired t-test $p<0.01$ ). The number of inactive nose pokes was much lower (figure 3.11A left, number of inactive nose pokes, black squares: WD 1, $7.7 \pm 0.9$ to WD $40,17.1 \pm$ $2.4 ; n=9$ ) and so was the number of saline active and inactive nose pokes (figure $3.11 \mathrm{~A}$ left, number of saline active nose pokes, open circles: WD 1, 10.2 \pm 5.4 to WD 40, 34.2 $\pm 8.7, n=$ 4; number of saline inactive nose pokes, open squares: WD 1, 3.0 \pm 1.7 to WD 40, 17.0 \pm 4.3 , $\mathrm{n}=4$ ). The rats injected with sh95 in the NAcSh also showed a similar pattern. The number of active nose pokes were much higher after prolonged withdrawal from cocaine SA (figure 3.11A right, number of active nose pokes, green circles: WD 1, 41.6 \pm 9.7 to WD $40,81.1 \pm$ $6.8 ; n=7$; paired t-test $p<0.01$ ). The number of inactive nose pokes after cocaine-SA and number of active as well as inactive nose pokes after saline-SA were also much lower for sh95 injected rats (figure 3.11 right, number of cocaine inactive nose pokes, black squares: WD $1,5.4 \pm 1.2$ to WD $40,13.3 \pm 3.7, n=7$; number of saline active nose pokes, open 
circles: WD 1, $7.6 \pm 1.5$ to WD 40, $19.7 \pm 2.9, \mathrm{n}=7$; number of saline inactive nose pokes, open squares: WD 1, $3.8 \pm 2.0$ to WD $40,7.9 \pm 1.7, \mathrm{n}=7$ ).

As the animals injected with sh95 AAV8 in the NAcSh were scored on the basis of the spread of the virus in the tissue, a correlation curve was plotted to check if a better spread of sh95 virus in the NAcSh would be negatively correlated with an increase in number of active nose pokes from WD 1 to WD 40 (figure 3.11B). However the value of the correlation coefficient is too close to zero to indicate any real correlation $\left(R^{2}=0.0087\right)$. This indicates that even the best spread of sh95 in the NAcSh tissue was not enough to impair incubation of cocaine craving.

Therefore the results mentioned so far indicate that AMPAR stabilization by PSD-95 governs the maturation of cocaine-induced silent synapses into CP-AMPAR containing synapses, which however, are NOT essential for maintaining drug-associated long-term memory or increase in the responsiveness of the animal to them. The data points towards the involvement of other brain regions and/or alternative mechanisms responsible for storage and maintenance of drug-related memories. It was indeed surprising to see that striking cellular changes in the NAcSh MSNs do not affect the drug-associated behavior of the animals, since NAc is known to be critical for mediating drug-related reward. 


\section{A Extinction tests}

WT + control



\section{WT + sh95}

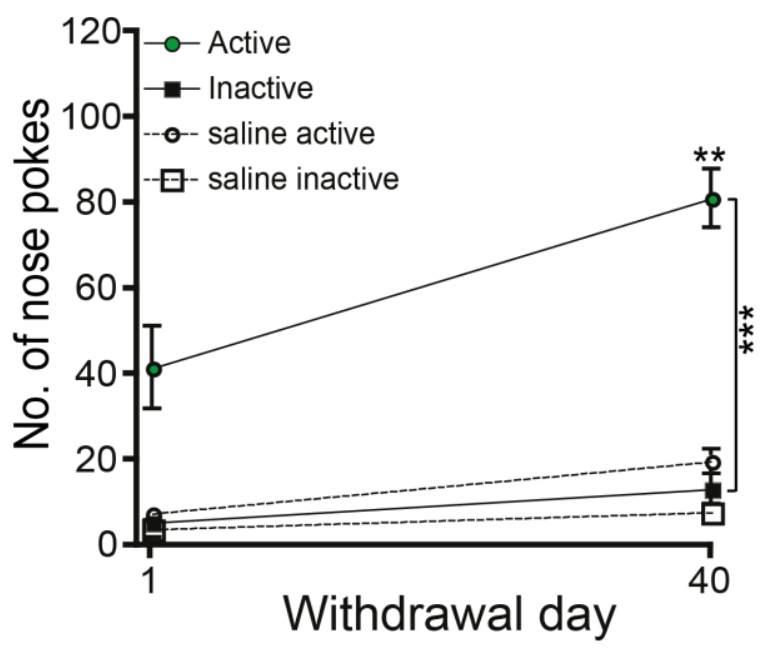

B



Figure 3.11 NAc-restricted knockdown of PSD-95 does not impair incubation of cocaine craving.

(A) $1 \mathrm{hr}$ extinction tests performed on rats after 40 days of withdrawal from cocaine self-administration (see Figure 3.9) resulted in significantly higher cue-induced cocaine seeking (as measured by number of active pokes) than on withdrawal day 1. Both groups of rats, injected with sh95 AAV8 or control virus have significant incubation of craving as indicated by increase in number of active nose pokes and difference between active and inactive pokes at withdrawal day 40. (B) Correlation graph between the quality of stereotaxic injection of sh95 AAV8 into in the rat NAc (as measured subjectively as a score of infection spread in the tissue) and increase in active nose pokes. Pearson correlation coefficient $R^{2}=$ 0.0087 , indicating there is no correlation. 


\subsection{Cocaine induces silent synapses via the thrombospondin receptor $\alpha 2 \delta 1$}

In the previous sections it was described that cocaine-CPP leads to the induction of silent synapses in the NAcSh. It has been shown previously that simply in vivo exposure to cocaine by means of 5 daily experimenter-administered cocaine injections is enough to induce silent synapses in the NAcSh (Huang et al., 2009). The previous sections focused on what could be the fate of the silent synapses, and how their maturation could influence long-term drugassociated behavior. However an extremely pertinent question remains unanswered - how are these silent synapses induced by cocaine? Which mechanisms are responsible for thus rejuvenating an adult brain region with new born synapses ready for further plasticity?

I addressed this question in collaboration with Prof. Yan Dong at University of Pittsburgh, USA. Together we aimed to test whether thrombospondins (TSPs) which are astrocytesecreted synaptogenic proteins, are involved in the process of inducing new silent synapses upon cocaine exposure (Risher and Eroglu, 2012). The voltage gated calcium channel subunit $\alpha 2 \delta 1$ has been identified as the thrombospondin receptor (Eroglu et al., 2009). Since the analgesic drug gabapentin also has high affinity for a2ס1 (Gee et al., 1996), it can block the TSP- $\alpha 2 \delta 1$ association thereby blocking formation of new synapses (Eroglu et al., 2009). The lab of our collaborator Prof. Yan Dong, at the University of Pittsburgh, PA, USA tested and confirmed that indeed gabapentin can also block generation of silent synapses in adult rats after exposure to cocaine (data unpublished). As systemic gabapentin injections could potentially react with unwanted targets, I tested the involvement of a2ס1 receptor in the generation of cocaine-induced silent synapses in adult mice, using an RNAi approach, targeted specifically to $\alpha 2 \delta 1$. The shRNA targeting $\alpha 2 \delta 1$ was packaged into a lentiviral vector co-expressing GFP as a marker and stereotaxically injected into the NAcSh of mice (figure 3.12 A). The virus was then allowed to express and mediate the knock-down of $\alpha 2 \delta 1$ in the NAcSh for 2-3 weeks after which one i.p injection of cocaine was administered to the mice in its home cage for 5 consecutive days. On the $6^{\text {th }}$ day, which was at 1 day of withdrawal from cocaine, acute slices of the NAcSh were prepared and minimum stimulation electrophysiological assay was performed on GFP positive or infected NAcSh cells (figure 3.12A,C). Vehicle controls were mice injected with the same $\alpha 2 \delta 1$ shRNA lentivirus but given 5 consecutive saline injections instead of cocaine (figure 3.12B). As an injection control, mice were injected with lentivirus containing a shRNA for luciferase and 2-3 weeks after, administered cocaine injections over 5 consecutive days (figure 3.12D). The baseline number 
of silent synapses in the NAcSh upon knock-down of $\alpha 2 \delta 1$ was found to be $22.97 \% \pm 8.68 \%$ (figure 3.12E, grey, $n / m=6 / 22$ ). Upon cocaine treatment to the mice injected with the control virus (luciferase NAcSh knockdown), the number of silent synapses were double that of the baseline (figure $3.12 \mathrm{E}$, black, $51.47 \% \pm 5.50 \%, \mathrm{n} / \mathrm{m}=6 / 19$ ). However cocaine treatment failed to induce silent synapses upon knockdown (KD) of $\alpha 2 \delta 1$ in the NAcSh (figure 3.12E, green, $25.71 \% \pm 6.69 \%, \mathrm{n} / \mathrm{m}=6 / 18$; one way ANOVA with all conditions, $\mathrm{F}_{2,15}=4.935$, $p<0.05$; $\alpha 2 \delta 1-K D$ saline vs. $\alpha 2 \delta 1-K D$ cocaine, post hoc Bonferroni test $p>0.05$; $\alpha 2 \delta 1-K D$ cocaine vs. luciferase-KD cocaine, post hoc Bonferroni test, $p<0.05)$. Therefore the knockdown of a2ס1 completely blocks the induction of silent synapses upon cocaine exposure as the number of silent synapses remains close to baseline. This result shows that $\alpha 2 \delta 1$ is required for the process of generating new silent synapses upon in vivo cocaine exposure. As a2ס1 is the receptor for thrombospondins it is also highly likely that these astrocyte secreted proteins are involved in the synaptogenic signals that induce new silent synapses upon in vivo exposure to cocaine. Therefore a potential thrombospondin - $\alpha 2 \delta 1$ pathway has been recognized for generation of cocaine-induced silent synapses in the adult brain. This can be studied in future experiments after establishing thrombospondin $\mathrm{KO}$ mouse lines. 
A



B



$+40 \mathrm{mV}$

D

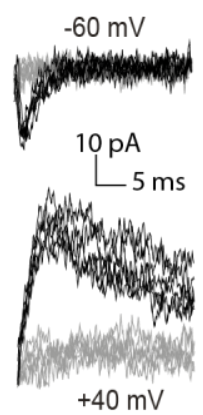

a2ס1-KD saline
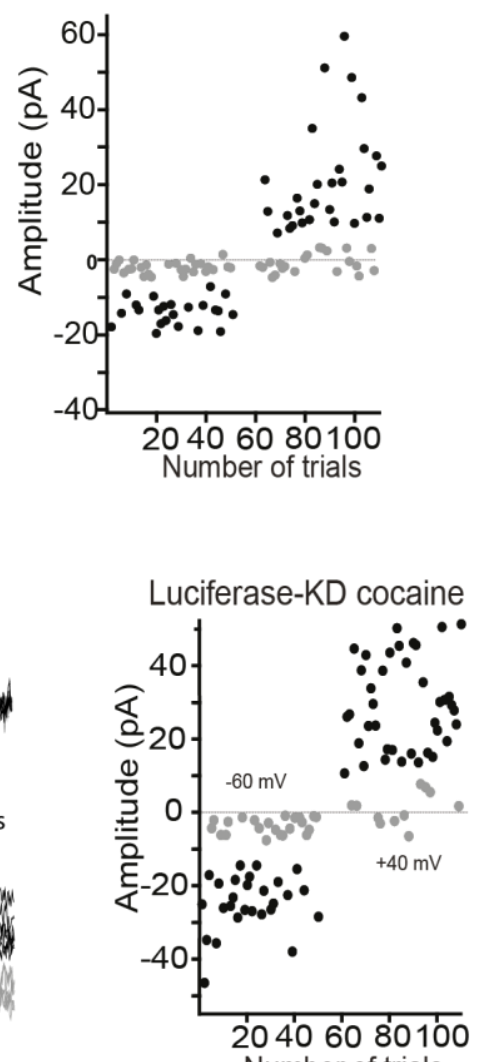

C
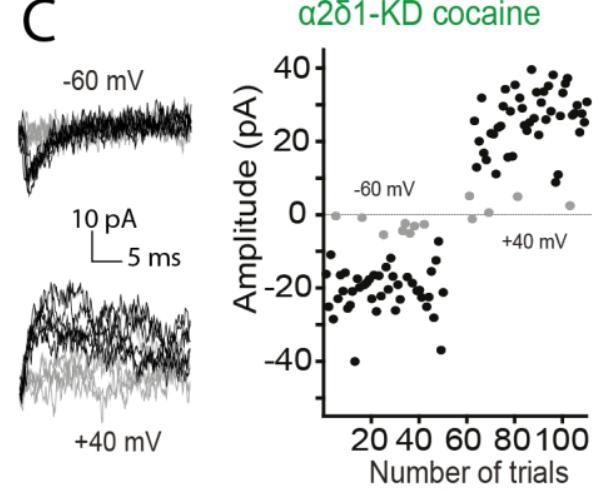

E



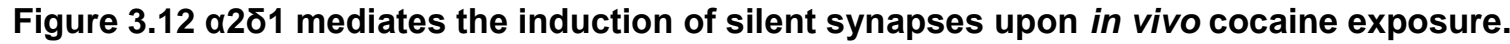

(A) Timeline of the stereotaxic injection of lentivirus into the mouse NAcSh followed by 5 consecutive injections of cocaine and electrophysiology from infected cells on withdrawal day 1. (B), (C) and (D) EPSCs evoked at $-60 \mathrm{mV}$ and $+40 \mathrm{mV}$ by minimum stimulation (left side) over 100 trails from an example recording from saline treated $\alpha 2 \delta 1-K D$ (knockdown) mice $(B)$, cocaine treated $\alpha 2 \delta 1-K D$ mice (C) and cocaine treated luciferase-KD mice (D) at withdrawal day 1. (E) Summarized results showing that cocaine induces a high number of silent synapses in luciferase-KD control mice compared to the baseline of saline treated $\alpha 2 \delta 1-K D$ mice. However $\alpha 2 \delta 1-K D$ completely blocks the induction of silent synapses upon cocaine treatment. $\mathrm{n} / \mathrm{m}$ is indicated in the figure below the respective data points. 
4. Discussion 


\section{Discussion}

It is known from previous studies that the mesolimbic dopamine system that projects from the VTA to the NAc, is the major substrate of neural adaptations underlying reward and reinforcement for addictive drugs (Robbins and Everitt, 1996b; Hyman and Malenka, 2001; Kauer and Malenka, 2007). All drugs of abuse effectively increase dopamine transmission at the NAc, and the glutamatergic transmission at NAc MSNs is of particular importance in the reward circuit. It has been widely reported that drugs of abuse such as cocaine, cause maladaptive plasticity in glutamatergic signaling of the brain's reward pathway thereby hijacking the normal synaptic plasticity mechanisms and controlling drug-seeking behavior (Hyman et al., 2006b; Kalivas, 2004; Kourrich et al., 2007; Lee and Dong, 2011; Lee et al., 2013; Ma et al., 2014).

In this study, I used retention of the preference learned during the cocaine-CPP assay as a measure of drug-associated memory. To decipher the cellular basis of this long-term memory, I studied cocaine-induced synaptic plasticity in the NAcSh MSNs. The results of this study indicate that silent synapses that are induced in the NAcSh upon cocaine exposure, mature into CP-AMPAR containing synapses if the animal learns to associate the drugexperience with the environmental cues provided by the CPP training. However the results also show that this process of maturation of NAcSh silent synapses to CP-AMPAR-bearing synapses is NOT an absolute requirement for long-term retention of drug-associated memory. Additionally, this study shows that the mechanism of generation of silent synapses in NAcSh upon cocaine exposure, involves the role of the thrombospondin receptor $\alpha 2 \delta 1$.

\subsection{The association learnt by the mice in the cocaine-CPP paradigm becomes a robust long-term memory}

The results described in section 3.1 and figure 3.1 clearly show that mice learn to associate the rewarding experience of cocaine with the particular environment or chamber where they were conditioned to experience it. This directly translates to longer amount of time spent in that particular environment and thus increases the place preference score (PPS). Wild type mice not only learn this association well, as inferred by the increase in the PPS at withdrawal day 1 (WD 1) but also remember this association even after prolonged withdrawal from cocaine-CPP (observed till WD 45). This indicates that the memory associated with the 
drug-experience is long-lasting and robust. Therefore, this model successfully represents the cue-related long-term memory that human drug-subjects bear, which often causes them to relapse after prolonged abstinence from the drug, upon encountering drug-related cues.

It should be noted that all measures were taken to avoid causing 'extinction' of drugassociated memory in the mice. The loss of performance that occurs when a Pavlovian signal is no longer paired with a reinforcer is termed as extinction (Bouton, 2002; Self et al., 2004). Therefore for the CPP assay, all the tests were done under 'extinction' conditions as the mice were allowed to explore the CPP apparatus (the environmental cues or Pavlovian signal) without being under the effect of cocaine, the reinforcer. Therefore a number of CPP tests performed repeatedly in close succession would decrease the preference (or PPS score) of the mice for the cocaine-paired chamber (Mueller and Stewart, 2000). That is why on every animal a maximum of 3 CPP tests were performed with a substantial time gap - the pre-test for the initial preference, the test on WD 1 to measure learning of the CPP preference and if kept for long-term withdrawal a final test to measure retention of the CPP preference. All the cohorts of wild type mice on an average show a highly positive preference for the cocainepaired chamber at every withdrawal time point tested, therefore extinction of the CPP preference was NOT observed by conducting these 3 CPP tests. This was the reason for keeping separate cohorts of WD 10-15, WD20-25, WD 30-35 and WD 40-45 instead of using the same mice for repeated CPP tests at the various withdrawal days.

\subsection{Cocaine induces silent synapses in the NAcSh which mature during withdrawal from cocaine}

It is a fairly recent finding that in vivo exposure to cocaine generates AMPA-silent synapses in the adult NAcSh (Huang et al., 2009). The few studies which have shown this phenomenon including this one, use a robust assay to estimate the proportion of silent synapses in a given cell population - minimum stimulation protocol followed by failure analysis (Brown et al., 2011; Huang et al., 2009; Koya et al., 2012; Lee et al., 2013; Ma et al., 2014). The results of this study also clearly show that the number of silent synapses in the NAcSh approximately doubles following 5 cocaine i.p injections with (figure 3.2F) or without (figure 3.12E) CPP. It has been shown that these silent synapses are in fact 'new' and generated by insertion of NR2B-containing NMDARs into new synaptic sites (Brown et al., 2011; Huang et al., 2009) instead of being formed by AMPAR internalization at existing 
synapses. In fact, structural plasticity, in terms of increase in number of dendritic spines and dendritic arborization in the NAc MSNs, has long been known to be induced by repeated cocaine experience (Robinson and Kolb, 1999, 2004; Robinson et al., 2001). Therefore it is possible that newly-formed silent synapses induced by cocaine-exposure, account for the observed morphological alterations of increase in spine density.

\subsection{Silent synapses of the NAcSh mature into CP-AMPAR containing synapses, when animals learns to associate environmental cues to the rewarding experience of cocaine}

In this study I wished to address the fate of the silent synapses that are generated upon cocaine exposure. There is previous evidence that the number of silent synapses decreases during withdrawal from cocaine (Huang et al., 2009). Synaptic pruning could account for the 'disappearance' of these silent synapses, much like the pruning of developing nascent synapses or refinement of pre-existing circuits (Ann M. Lohof, 1996). However I asked whether a large number of cocaine-induced silent synapses in the NAc might eventually be maturing in to non-silent synapses, by incorporation of AMPARs.

To examine the kinetics of the maturation of silent synapses generated in adult animals upon cocaine exposure, I performed a time course experiment by measuring the proportion of silent synapses in NAcSh MSNs at varying time points of withdrawal from cocaine-CPP (figure 3.2F circles). As described in section 3.2, the proportion of these silent synapses was seen to be lowered at 10-15 days of withdrawal from cocaine and subsequently reached baseline by WD 20. At the same time, to check if AMPAR incorporate into silent synapses induced by cocaine I measured the rectification index to calculate the proportion of CPAMPARs, which have long been known to be important for various forms of synaptic plasticity.

The results described in section 3.2 (and figure 3.2F, squares) shows that CP-AMPARs slowly accumulate in NAcSh synapses during the course of withdrawal from cocaine-CPP peaking at WD 30-35. This study shows that CP-AMPARs accumulate in NAcSh synapses following a protocol involving passive administration of cocaine. Previously, it was shown that only contingent procedures like self-administration (SA) of cocaine result in CP-AMPAR accumulation in the NAc and not experimenter-administered cocaine (McCutcheon et al., 
2011a). Although one study did show that 10 consecutive passive injections of cocaine increases rectification index in 4-6 week old mice after 35 days of withdrawal from cocaine, CP-AMPARs are largely considered as a hallmark of incubation of craving following cocaine self-administration (Conrad et al., 2008; Grimm et al., 2001; McCutcheon et al., 2011a; Pickens et al., 2011). It has been proposed that the incorporation of these GluA2-lacking AMPARs which also have higher conductance (as compared to calcium impermeable AMPARs) increases the responsiveness of NAc MSNs to glutamatergic inputs from cortical and limbic regions, thereby mediating incubation of craving (Conrad et al., 2008). However by increasing the reactivity of NAc MSNs to cocaine-associated cues, the accumulated CPAMPARs may be responsible for other aspects of drug-associated behavior as well.

In this study, when cocaine was passively injected into mice in their home cage, without being subjected to the CPP assay, CP-AMPAR were NOT found to be elevated in the NAcSh (figure 3.3, orange). Therefore it is the association learnt during the CPP assay, which makes a difference relative to passive administration of cocaine and is responsible for inducing CPAMPAR accumulation in the NAcSh. This result shows that not just self-administration of the drug, but even the association of the non-contingent drug experience to salient cues, is enough for CP-AMPAR accumulation in the NAcSh. It can be inferred from this data that CPAMPAR accumulation and transmission in the NAc upon prolonged withdrawal from a drugexperience is a relatively more general hallmark of drug-associated behaviors.

The kinetics of incorporation of CP-AMPARs in the NAcSh post cocaine-CPP might differ from what occurs after cocaine SA. It has been shown that CP-AMPAR-mediated transmission in the NAc remains elevated till up to 70 days of withdrawal from cocaine SA (Wolf and Tseng, 2012). However the results of this study show that CP-AMPAR-mediated transmission peaks around 30 days of withdrawal from cocaine-CPP, after which it was seen to decrease again (figure $3.2 \mathrm{~F}$ squares). Thus it is possible that different routes of administration of the same drug cocaine, have varied effects on the duration of drug-induced plasticity. The results of this study exemplify that though CP-AMPARs incorporate into NAcSh synapses upon passive administration of cocaine accompanied by the CPP paradigm, this plasticity is relatively short-lived compared to that seen after cocaine SA wherein, long-lasting CP-AMPARs in the NAc are thought to mediate incubation of craving.

The results described in section 3.2.1 show that the CP-AMPAR incorporation is a gradual process that takes place due to prolonged withdrawal and it is NOT a plasticity mechanism that can be induced in a short time period by exposure to drug-associated cues. When the electrophysiology was done by omitting the step of CPP behavior test, in order to prevent 
exposure to drug-associated cues (the CPP apparatus), CP-AMPARs could still be detected from the NAcSh MSNs (figure 3.3, red). Moreover, a prolonged period of withdrawal was shown to be absolutely required in order for this gradual plasticity to take place. Mice that were subjected to a 30-day long cocaine-CPP protocol did not have accumulated CPAMPARs in the NAcSh one day later, although the time passed since the first cocaine injection was around 30 days. Therefore CP-AMPARs were shown to peak 30 days after cocaine-CPP (figure 3.2F squares), a process which occurs only upon withdrawal and is NOT just dependent on time passed since the first drug exposure.

Furthermore, when the kinetics of silent synapse maturation and CP-AMPAR accumulation during withdrawal from cocaine-CPP was compared, it was found to be strikingly complementary (figure 3.3). As the silent synapse number decreases, the proportion of CPAMPARs in the NAcSh increases. This suggested that silent synapses may be maturing by incorporation of CP-AMPARs. Systemic injections of SYN119, a mGluR1 positive allosteric modulator have been shown to lower the rectification index of (hence remove CP-AMPAR from) NAc MSNs at long-term withdrawal from cocaine-SA (Loweth et al., 2014b). mGluR1 activation has been shown to remove CP-AMPARs from NAc synapses (Loweth et al., 2014a, 2014b; McCutcheon et al., 2011b) probably via a form of mGluR-LTD (Bellone and Lüscher, 2006). Therefore I administered SYN119 systemically, via i.p. injection to mice after 20-30 days of withdrawal from cocaine-CPP as a high number of CP-AMPAR would be accumulated in the NAcSh at that time point (figure 3.2F squares). As expected, this resulted in low rectification index as compared to the vehicle controls, which indicates that SYN119 could mediate CP-AMPAR removal via mGluR1 (section 3.3 and figure 3.4F, squares). This indeed resulted in the re-silencing of NAcSh synapses, as the proportion of silent synapses measured after SYN119 injection was much higher than the vehicle control (figure 3.4F, circles). In fact SYN119 action created 50\% silent synapses in the NAcSh which is very similar to the number of silent synapses observed at the starting point, directly after 5 i.p injections of cocaine (figure 3.2F, grey circle and figure 3.12E, black circle). This indicates that mGluR1 activation via SYN119, leads to full recovery of cocaine-generated silent synapses, to the level observed on withdrawal day 1. This experiment demonstrates that NAcSh synapses can be re-silenced by removal of the CP-AMPARs that accumulate during prolonged withdrawal from cocaine-CPP. This serves as correlative evidence that CPAMPARs incorporate into the same silent synapses that are induced by cocaine, as their removal leads to re-silencing of the synapses. A previous study using a pharmacological block of CP-AMPARs with Naspm, on basolateral amygdala to NAcSh synapses at 45 days 
of withdrawal from cocaine SA, has shown the partial reemergence of silent synapses (Lee et al., 2013). This highlights the importance of mGluR1 tone at NAcSh synapses for druginduced plasticity.

However, it was noteworthy that, the long-term memory of the association learnt in the CPP assay remained intact in the animal even when the accumulated CP-AMPARs were removed and the NAcSh synapses were therefore silent (figure 3.4B). It could however be a possibility, that only one systemic injection of SYN119 might not be enough to abolish a robust memory trace which once formed, may be maintained by multiple brain regions.

The results discussed so far indicate that cocaine-induced silent synapses in the NAcSh mature by incorporation of CP-AMPARs. Since, CP-AMPAR incorporation into NAc has been shown to be critical for development of incubation of cocaine craving, it was interesting to test whether the maturation of NAcSh silent synapses to CP-AMPAR containing ones is essential for other drug-associated behaviors.

\subsection{MAGUKs are essential for maturation of silent synapses but not for retention of drug-associated memory}

Since the DLG-MAGUK protein PSD-95 has been shown to play a crucial role in AMPA receptor retention at glutamatergic synapses (Ehrlich and Malinow, 2004; Schlüter et al., 2006), a former colleague in the lab (Dr. Anna Suska) tested PSD-95 KO mice as described in section 3.4. The behavioral and cellular phenotype of PSD-95 KO mice was distinct from that of WT mice. The results implied that in the absence of PSD-95, AMPAR trafficking and retention at the glutamatergic synapses of NAcSh is affected because even the baseline number of silent synapses were found to be higher in PSD-95 KO mice than in WT mice (figure 3.7). This result is concomitant with earlier findings wherein PSD-95 was implicated to play an important role in maturation of silent or nascent synapses during development (Béïque et al., 2006; Huang et al., 2015a). In spite of many silent synapses in the NAcSh, the PSD-95 KO mice were nevertheless capable of learning the association in the cocaine-CPP paradigm very well, and more NAcSh synapses could be rendered silent by cocaine exposure. The most striking finding was that after 20-25 days of withdrawal from cocaineCPP, the PSD-95 KO mice completely forget the association they had managed to learn well during the CPP training. This was accompanied by a cellular phenotype in the NAcSh, wherein unlike WT animals, CP-AMPARs did NOT accumulate in the MSNs after prolonged 
withdrawal from cocaine-CPP. This data showed that PSD-95 is essential for CP-AMPAR incorporation into silent synapses during withdrawal. It also suggested that in the absence of stable CP-AMPAR incorporation into NAcSh, owing to the lack of PSD-95, the long-term memory retention of drug-associated cues could be impaired. It was therefore necessary to test whether CP-AMPAR incorporation into NAcSh MSNs was essential for long-term retention of drug-associated memory.

The DLG-MAGUK, SAP102, is predominantly present in the PSD during early development and has been shown to compensate for the lack of PSD-95 (Bonnet et al., 2013; Cuthbert et al., 2007; Elias et al., 2006). Similar behavioral and electrophysiological assays were performed using SAP102 KO mice (section 3.5) to test its potential role in the maturation of cocaine-induced silent synapses to CP-AMPAR containing synapses. The cellular phenotype of SAP102 KO and PSD-95 KO was found to be similar. Although SAP102 $\mathrm{KO}$ mice did not have a high number of baseline silent synapses(figure 3.7), cocaine-CPP induced silent synapses, also in SAP102 KO mice as they learnt the association of the CPP paradigm. After long-term withdrawal too, the NAcSh silent synapses did NOT mature and did NOT have significant incorporation of CP-AMPARs, but this however was not enough to impair their long-term drug-associated memory retention (figure 3.6)! Therefore although cellular phenotype of SAP102 KO and PSD-95 KO was very similar, the long-term behavioral phenotype was markedly different. Thus both PSD-95 and SAP102 are required for the conversion of silent synapses to CP-AMPAR containing synapses. This is consistent with previous results showing the role of PSD-95 in maturation of developing synapses and preferential incorporation of GluA2-lacking AMPARs (Ehrlich and Malinow, 2004) as well as functional interplay of SAP102 and PSD-95 (Bonnet et al., 2013). However the key difference shown by this study is that while PSD-95 is required for the long-term CPP memory retention, SAP102 is not.

Since this study was focused on the maturation of cocaine-induced silent synapses to CP-AMPAR bearing synapses specifically in the NAcSh, I did a NAcSh-restricted knock-down of PSD-95 to delineate the specific role of PSD-95 in the NAcSh, in the long-term retention of drug associated memory. The results showed that PSD-95 in the NAcSh alone is not sufficient for mediating long-term retention of the CPP memory, because when it is knocked down, the animal can still remember its preference to the cocaine-paired chamber (figure $3.8 \mathrm{C}$ blue). I confirmed that at the cellular level in the NAcSh, knockdown of PSD-95 in NAcSh MSNs produced a phenotype similar to that of the PSD-95 KO animal (figure 3.9 blue). A compensatory function performed by SAP102 in the cells lacking PSD-95 in the 
NAcSh, could still be a potential explanation for the lack of a behavioral phenotype. Therefore by creating a 'double knock-out' like situation for PSD-95 and SAP102 in the NAcSh, I tested whether PSD-95 and SAP102 both act in concert to facilitate stable CP-AMPAR retention at NAcSh synapses which might govern some of the drug-related behaviors of the animal. This led to an even higher number of silent synapses than the single PSD-95 knock down, that did not mature over the period of long-term withdrawal from cocaine-CPP (figure 3.9G). This and the lack of CP-AMPAR incorporation after long-term withdrawal in the 'double knock-out' like MSNs clearly demonstrates that the two MAGUKs, PSD-95 and SAP-102 are both involved in maturation of cocaine-induced silent synapses to CP-AMPAR bearing synapses in the NAcSh. However the lack of accumulated CP-AMPARs in the NAcSh did not impair the longterm retention of drug-associated memory (figure $3.8 \mathrm{C}$ green). Thus all the above experiments indicate that maturation of cocaine-induced silent synapses to CP-AMPAR containing synapses, is facilitated by PSD-95 and SAP102, but is NOT responsible for the long-term retention of drug-associated memory. It could be possible that enough number of MSNs could not be infected for sufficient PSD-95 knockdown in the NAcSh, however this unlikely given the spread of the virus in the tissue and the high infection rate (figure 3.8B). Moreover, a similar dissociation of the cellular and behavioral phenotype was observed upon SYN 119 systemic injection into WT (section 3.4) as well as in the SAP102 KO mice at longterm withdrawal from cocaine-CPP.

\subsection{PSD-95 in the NAcSh is unlikely to be required for incubation of cocaine craving}

In this study, systemic mGluR1 activation and NAcSh-specific knockdown of PSD-95, resulted in blockade of CP-AMPAR accumulation at the NAcSh synapses, but did NOT impair retention of long-term cue-associated CPP memory (section 3.3, 3.6 and 3.7). However, although the CP-AMPARs which accumulate in the NAcSh during withdrawal from cocaineCPP do NOT mediate long-term retention of drug associated memory, they could nevertheless be responsible for mediating the intensity of a behavioral response to drugassociated cues. Such a behavioral response was studied using the incubation of craving model wherein prolonged withdrawal from cocaine SA leads to elevated cue-induced cocaine seeking in rodents (Grimm et al., 2001; Pickens et al., 2011). The results of this study (described in section 3.8) showed that rats with NAc-restricted knockdown of PSD-95 
displayed incubation of cocaine-craving to a similar extent as the control injected animals (figure 3.11). It is important to note that CP-AMPARs in the NAcSh do NOT mediate the longterm retention of drug-associated memory, in the case of CPP as shown before, as well as in the case of self-administration, as the rats in principle remember the pairing of the active nose poke to the cocaine reward. However it was a surprising finding that the lack of accumulated CP-AMPARs in the NAcSh, did NOT affect the intensity of the behavioral response of the animal to cue-induced cocaine seeking.

CP-AMPAR incorporation into the NAc neuronal circuit can potentially alter synaptic properties owing to activation of intracellular calcium-coupled signaling and the higher single channel conductance of CP-AMPARs (Liu and Zukin, 2007). The accumulation of CPAMPARs in NAc synapses after prolonged withdrawal from cocaine self-administration (SA) has been shown to mediate incubation of cocaine craving (Conrad et al., 2008). In the latter study showed that intra-NAc core injections of Naspm, a selective CP-AMPAR antagonist, significantly impaired incubation of cocaine craving. It was subsequently shown that reducing CP-AMPAR transmission in the NAc by using mGluR1 positive allosteric modulators can attenuate incubation of cocaine craving following cocaine SA (Loweth et al., 2014b). Furthermore, it has been shown that removal of CP-AMPARs from NAc synapses in a pathway-specific manner after prolonged withdrawal from cocaine SA, via in vivo LTD, resilences the synapses and impairs incubation of cocaine craving (Lee et al., 2013; Ma et al., 2014).

In this study, it is possible that enough number of NAcSh MSNSs could NOT be targeted using the stereotaxic injections of sh95 (shRNA against PSD-95) in rats. Indeed the spread of the viral infection was smaller as compared to that seen in mouse NAcSh, owing to the larger size of the brain and hence larger NAcSh region in the rat (compare 3.10B to 3.8B). Efforts were made to increase the volume of virus solution injected into the NAcSh of rats, however this failed to improve the spread of the virus in the NAcSh tissue (data not shown). To check if a better spread of the virus injection led to weaker behavioral responses by the animals, a correlation graph was plotted between the increase in nose-poking after prolonged withdrawal from cocaine-SA (indicating incubation of cocaine craving) and the NAcSh area transduced with sh95 (figure 3.11B). This graph showed NO trend indicating that more number of sh95transduced MSNs would lead to more impairment in incubation of cocaine craving.

Recent evidence indicates that another type of silent synapses can be induced upon in vivo cocaine exposure, which can be detected at 6-11 days of withdrawal from cocaine, upon administering a test or priming injection of cocaine. These silent synapses were shown to be 
preferentially induced ONLY in MSNs of the NAcSh which were strongly activated post cocaine exposure, as indicated by the Fos activation marker. These sparsely-distributed neurons which comprise of only $2-3 \%$ of the total neuronal population of the NAc, were thought to mediate psychomotor sensitization (Koya et al., 2012). Subsequently select neuronal ensembles of Fos activated MSNs in the NAc shell, have also been implicated in mediating context-induced re-instatement of cocaine seeking that occurs when cocaine SA is followed by extinction training in a novel environment (Cruz et al., 2014). Although recent reports claim that silent synapses are formed only in such Fos-activated neuronal ensembles that pertain to associative learning (Whitaker et al.), the data in this study and in previous studies have shown that cocaine-induced silent synapses can be readily detected from randomly selected cells of the NAcSh post in vivo cocaine exposure (Huang et al., 2009; Brown et al., 2011; Lee et al., 2013; Ma et al., 2014). It may however be possible, that these few cells of the NAcSh that form fos-activated neuronal ensembles might be critically important for subsequent steps of synaptic plasticity which contribute to development of incubation of craving. This could suggest that presence of a small number of critical MSNs may be sufficient for the overall drug-induced behavior after prolonged withdrawal. Therefore in this study, it is possible that some MSNs belonging to a critical neuronal ensemble were either left untargeted by the viral injections or they might bear an alternative mechanism to maintain the same behavioral response of the animals, despite changes at the cellular level in majority of the NAcSh neurons.

It is noteworthy that recent studies have shown that removal of CP-AMPARs accumulated in the NAc synapses following withdrawal from cocaine SA, can affect incubation of craving in a pathway-specific manner as dissected by optogenetic approaches (Lee et al., 2013; Ma et al., 2014). These studies indicate that the glutamatergic projections from basolateral amygdala as well as those from the infralimbic region of the pre-frontal cortex to NAc shell serve as 'pro-relapse circuitry', that increase incubation of craving. On the other hand, glutamatergic projections from the pre-limbic region of the pre-frontal cortex to the NAc core, function to oppose incubation of craving and constitute the 'anti-relapse' circuitry. It is therefore possible that multiple mechanisms counter each other to produce the 'net' behavioral response from the animal after prolonged withdrawal from cocaine SA. In this study however, no particular glutamatergic pathway was targeted and therefore glutamatergic afferents from pre-frontal cortex, basolateral amygdala and ventral hippocampus could all have been affected to varying degrees by electrical stimulation. Thus it is not possible to determine the relative contribution of each of these pathways towards the incubation of 
craving in this study. It may well be that lack of PSD-95 and hence the block of maturation of cocaine-induced NAcSh silent synapses to CP-AMPAR containing synapses, affects the different glutamatergic pathways to NAcSh in an anti or pro-relapse manner, to varying extents. Thus the possibility of such parallel antagonizing mechanisms countering each other with the result that 'net' behavior remains unchanged, cannot be ignored.

Although the results of this study indicate that CP-AMPAR accumulation in the NAcSh after long-term withdrawal from cocaine is a general signature of drug-induced synaptic plasticity, it may not be essential for drug-related behavior per se. The approaches that block CP-AMPARs with indirect approaches such as mGluR1 activation and optogenetic in vivo LTD to remove CP-AMPARs (as opposed to a specific pharmacological blocker such as Naspm), may also be having another common effect which may be responsible for alterations in the extent of incubation of craving. Hence it could still be a possibility that removal of CP. AMPARs is a secondary effect of this manipulation and not causal for changes in drug-related behavior.

Another detail to consider is that the manipulations used in this study targeted the shell region of the NAc specifically. Intravenous cocaine was shown to increase extracellular dopamine levels preferentially in the NAc shell, as compared to the NAc core (Pontieri et al., 1995; Rodd-Henricks et al., 2002). However these two regions of the NAc - shell and core, are both known to have differential effects on drug-seeking behavior (Di Ciano et al., 2007; Fuchs et al., 2004; Ito et al., 2004). Some studies report that the core region of the NAc is more important for cue-induced cocaine seeking and that CP-AMPAR expression is more pronounced in this region (Conrad et al., 2008; Everitt and Robbins, 2005). Indeed many of the reports show that specifically targeting the NAc core with Naspm and mGluR1 positive allosteric modulators suppresses incubation of cocaine craving (Conrad et al., 2008; Loweth et al., 2014b) while other studies show that optogenetically targeting specific pathways to the NAc shell for in vivo LTD can also cause a similar impairment (Lee et al., 2013). It may however be possible, that intracranial infusions of pharmacological drugs or in vivo LTD have a far wider spread of influence on the NAc tissue as compared to the specific RNAi-mediated knockdown in transduced MSNs that was used in this study. It is therefore possible that large regions of both shell and core of NAc are affected by strong manipulations such as intracranial infusion of Naspm or mGluR1 positive allosteric modulators which are used at substantially high dosages. In comparison, viral mediated transduction of MSNs which is limited to the NAcSh, is a weaker albeit more specific, manipulation to obtain NAcSh MSNs that lack accumulated CP-AMPARs after prolonged withdrawal from cocaine SA. Hence the 
effect of this manipulation may not have been enough to detect an overall change in the behavioral response of the animals to cue-induced cocaine seeking.

\subsection{Long-term retention of drug-associated memory may be coded by brain regions other than the nucleus accumbens}

There is enough evidence from previous studies which indicates that dopaminergic innervation of the dorsal striatum is important for establishing habitual or compulsive cocaineseeking (Ito et al., 2002, 2002; Vanderschuren et al., 2005; Yin et al., 2004). In fact a shift from goal-directed action to stimulus-response habit, is considered to be reflective of a shift in the control of drug-seeking behavior from ventral striatum (including the NAc) to the dorsal striatum (Belin and Everitt, 2008; Everitt and Robbins, 2005). Therefore during long-term withdrawal from cocaine-CPP, it is possible that the control of the drug-associated behavior that I observed had already shifted from ventral to dorsal striatum. This could explain why the synaptic plasticity that occurred in the NAc could not affect overall drug-associated animal behavior. Furthermore, the neural correlates of long-term drug-associated memory could also be formed in one or more of the cortico-limbic areas that project glutamatergic afferents onto the NAc, namely pre-frontal cortex, basolateral amygdala and ventral hippocampus (Lüscher and Malenka, 2011; Pascoli et al., 2014; Wolf, 2010). Indeed many studies have shown the importance of these individual brain regions in drug-seeking behavior (Lee et al., 2013; Ma et al., 2014; Stefanik and Kalivas, 2013; Stefanik et al., 2015). Perhaps using a similar approach of brain-region-restricted knock-down of PSD-95, in order to abolish the maturation of cocaine-induced silent synapses to CP-AMPAR containing synapses, in the aforementioned brain areas, could affect the retention of long-term drug associated memory. Therefore even though the NAc plays a key role in prioritizing emotional and motivational arousals for behavior, the drug-induced alterations in associated brain regions of the reward circuit may be critical for expression of drug-related behavior. 


\subsection{Role of the thrombospondin receptor $\alpha 2 \delta 1$ in generation of cocaine induced silent synapses : A new direction to explore thrombospondin-mediated adult synaptogenesis}

Along with determining the fate of cocaine-induced silent synapses in drug-associated behaviors, I also addressed the question of how cocaine induces silent synapses in the first place. I tried to explore the possible involvement of the thrombospondin (TSP) protein family in this process, as TSPs secreted by immature astrocytes, have been recently identified to be synaptogenic (Eroglu, 2009). I tested the involvement of the thrombospondin receptor, $\alpha 2 \delta 1$, a voltage-gated calcium channel (Eroglu et al., 2009), in the generation of cocaine-induced silent synapses in the NAcSh of adult mice. The NAcSh-restricted knockdown of $\alpha 2 \delta 1$ completely abolished the induction of silent synapses in this brain region following in vivo cocaine exposure (section 3.9, figure 3.12). Therefore this data shows that $\alpha 2 \delta 1$ receptor function is absolutely required for generation of silent synapses in an adult animal, in response to cocaine. Indeed a recent finding further supports this data, wherein $\alpha 2 \delta 1$ protein levels, as well as that of its ligand thrombospondin-1, were shown to be elevated in the NAc after cocaine SA followed by extinction training (Spencer et al., 2014). This study claims that $\alpha 2 \delta 1$ contributes to the development of cocaine-reinstated drug seeking and I speculate that this may occur through a mechanism of silent synapse generation in the NAc via the thrombospondin- $\alpha 2 \delta 1$ pathway of synaptogenesis. 


\subsection{Conclusions and outlook}

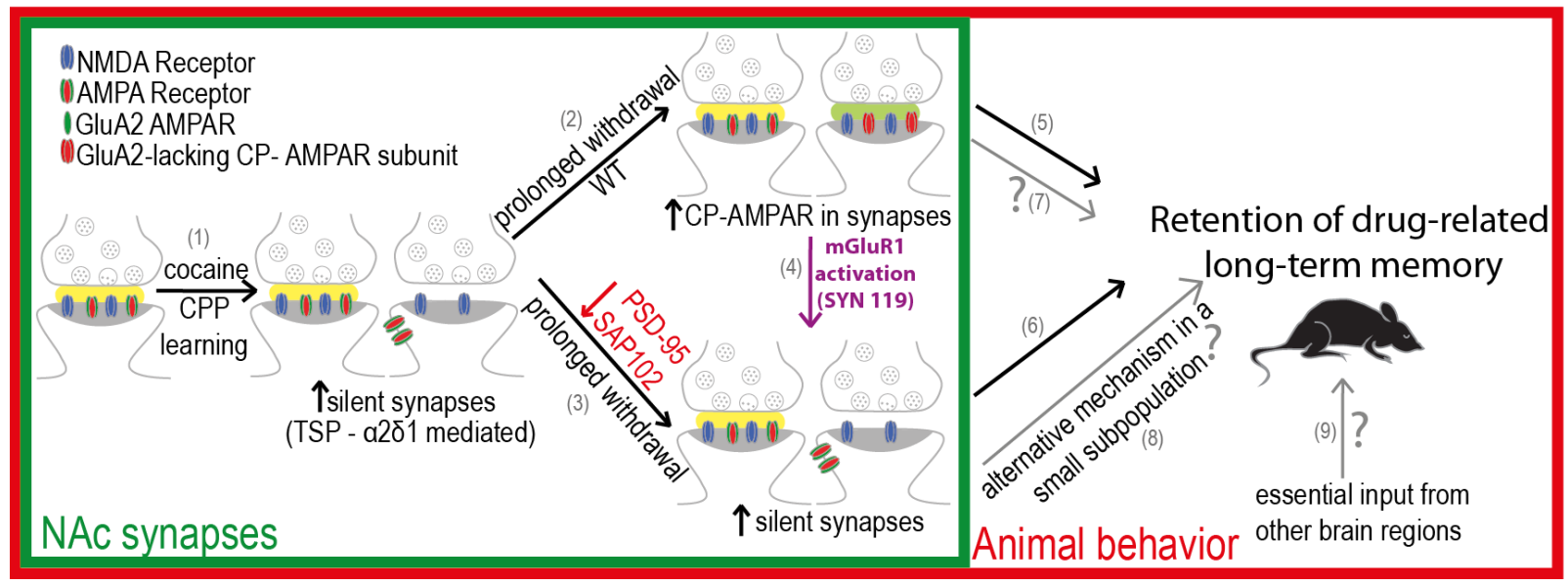

Figure 4.1 The dissociation between cocaine induced synaptic plasticity in the NAc (green box) and drug-associated long-term memory retention (red box).

(1) In vivo cocaine exposure leads to generation of AMPA-silent synapses in a process that may be mediated by interaction of thrombospondins with their receptor a2ठ1. (2) Prolonged withdrawal from cocaine-CPP results in maturation of the cocaine-induced silent synapses to CP-AMPAR containing synapses. (3) In the absence of MAGUK proteins - PSD-95 and SAP102, the cocaine-induced silent synapses do NOT mature and do NOT contain a higher proportion of CP-AMPARs. (4) mGluR1 activation can reverse the process of silent synapse maturation by removal of CP-AMPARs from the synapse, thereby re-silencing the NAc synapses. (5) In WT animals, the maturation of cocaine-induced silent synapses to CP-AMPAR containing synapses in the NAc shell, was accompanied by long-term retention of drug-associated memory as tested in the CPP paradigm. (6) In case of immature or resilenced synapses in the NAc shell, the behavioral phenotype remained unaltered as the animals could retain the long-term drug-associated memory. (7) It could be that the process of maturation of silent synapses and accumulation of CP-AMPARs could contribute in part to the long-term retention of drugrelated memory, however it is not fully responsible for it. (8) Alternative mechanisms may exist in select neuronal ensembles which enable the retention of drug-related memory despite a large number of silent synapses in the majority of NAc neurons. (9) Synaptic plasticity mechanisms induced by cocaine in other brain regions or in specific pathways of the mesocorticolimbic dopaminergic system can account for the unchanged overall behavior of animal despite cellular alterations in the NAc.

Schematic representations of the results obtained in this study, as well as possibilities that can be addressed in the near future are shown in figure 4.1. The electrophysiological assay of minimum stimulation performed on MSNs of the NAcSh clearly showed that cocaine-CPP as well as only i.p cocaine injections in mice can generate a large number of silent synapses (1). This process requires the functioning of $\alpha 2 \delta 1$, the receptor for thrombospondins (TSPs) astrocyte secreted synaptogenic glycoproteins. This is suggestive of an astrocyte-mediated mechanism for generation of silent synapses in adult animals upon in vivo cocaine exposure. To check if thrombospondin proteins are directly involved in this process, the TSP knock-out mice can tested to see if lesser silent synapses are generated upon cocaine exposure. Prolonged withdrawal from cocaine-CPP in WT animals resulted in the maturation of cocaineinduced silent synapses by incorporation of CP-AMPARs (2). CP-AMPARs have been 
thought to render NAc synapses more prone to relapse by mediating incubation of cocaine craving (Conrad et al., 2008). Since the CP-AMPARs in this study were shown to accumulate specifically due to the associative learning of the mice during the CPP paradigm, it was expected that they may be responsible for the concomitant long-term retention of the CPP memory (5). However a series of experiments using different approaches, proved otherwise. Using NAcSh-restricted RNAi mediated knockdown of PSD-95 as well as knock-out of SAP102, the NAcSh synapses were seen to be largely AMPA-silent and did not incorporate CP-AMPARs post withdrawal from cocaine-CPP (3). Furthermore, mGluR1 activation using systemic injections of SYN119, a mGluR1 positive allosteric modulator, resulted in resilencing of WT NAcSh MSNs by synaptic removal of CP-AMPARs that accumulated upon prolonged withdrawal from cocaine-CPP (4). Both of these scenarios resulted in a large number of immature synapses in the NAcSh that did NOT accumulate CP-AMPARs, however the animals could retain the long-term drug-associated memory as tested in the CPP paradigm (6). The only exception was found to be PSD-95 KO mice which did NOT retain the CPP memory after prolonged withdrawal and had a large proportion of silent synapses in the NAc which did not accumulate CP-AMPARs. Therefore it can be safely assumed that CPAMPARs in the NAcSh alone are not responsible for the long-term retention of drugassociated memory in the animal, although they may be partly involved in this process (7). It could be possible that only a small neuronal subpopulation in the NAcSh may bear the full potential to mediate long-term drug-related behavior as proposed by a few recent studies (Koya et al., 2012; Cruz et al., 2014; Whitaker et al.). It is possible that a mechanism other than maturation of silent synapses to CP-AMPAR bearing synapses, may be present in such critical neuronal ensembles that could account for the behavioral phenotype (8). Finally, it can be inferred that NAC alone does not play the major role in storage of long-term drugassociated memory after prolonged withdrawal from cocaine-CPP. Other brain regions of the mesocorticolimbic dopamine system that comprises the reward circuitry of the brain, could be responsible for the long-term retention of drug-related memory (9). 
5. Appendix 


\section{Appendix}

Parts of the experiments described in section 3 (results) were carried out by a former postdoc in the lab Dr. Anna Suska and some were done together with her. These experiments were part of joint efforts towards a common publication. The details of the work carried out by Anna Suska are specified in the table below. It should be noted that in this appendix I am ONLY acknowledging the contribution of other scientists and that all experiments.

Figure 3.1B $\quad$ WT CPP behavior - 16 mice of the saline WD 1 group, 21 mice of the cocaine WD 1 group and 18 mice of the cocaine WD 20 group by A.Suska.

Figure 3.2F $\quad$ WT Saline WD 1 group electrophysiology - minimum stimulation protocol in 5 animals and rectification index in 4 animals by A.Suska.

WT Cocaine WD 1 group electrophysiology - minimum stimulation protocol in 6 animals and rectification index in 6 animals by A.Suska.

WT Cocaine WD 20 group electrophysiology - minimum stimulation protocol in 6 animals and rectification index in 6 animals by A.Suska.

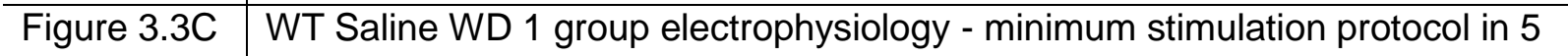
animals and rectification index in 4 animals by A.Suska.

WT home cage WD 20 group electrophysiology - rectification index in 5 animals by $A$.Suska.

WT w/o cue re-exposure group electrophysiology - rectification index in 6 animals by A.Suska.

\begin{tabular}{l|l}
\hline Figure 3.4F & $\begin{array}{l}\text { WT Saline WD } 1 \text { group electrophysiology - minimum stimulation protocol in } 5 \\
\text { animals and rectification index in } 4 \text { animals by A.Suska. }\end{array}$ \\
\hline
\end{tabular}

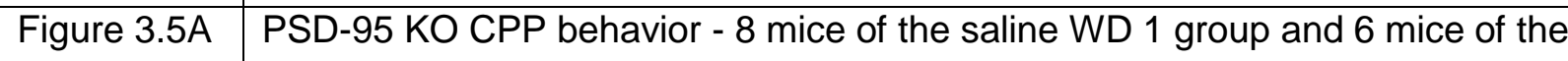
cocaine WD 1 and WD 20 group (5 out of the 6 mice) by A.Suska

Figure 3.5E $\quad$ PSD-95 KO Saline WD 1 group electrophysiology - minimum stimulation protocol in 6 animals and rectification index in 4 animals by A.Suska. PSD-95 KO Cocaine WD 1 group electrophysiology - minimum stimulation protocol in 4 animals and rectification index in 4 animals by A.Suska. PSD-95 KO Cocaine WD 20 group electrophysiology - minimum stimulation protocol in 5 animals and rectification index in 4 animals by A.Suska. 
\begin{tabular}{l|l} 
Figure 3.6A & SAP102 KO CPP behavior - 2 mice of the saline WD 1 group and 9 mice of the
\end{tabular} cocaine WD 1 and WD 20 group (5 out of the 9 mice) by A.Suska

\begin{tabular}{l|l} 
Figure 3.6E & SAP102 KO Saline WD 1 group electrophysiology - minimum stimulation
\end{tabular} protocol in 4 animals and rectification index in 3 animals by A.Suska.

SAP102 KO Cocaine WD 1 group electrophysiology - minimum stimulation protocol in 4 animals and rectification index in 4 animals by A.Suska.

SAP102 KO Cocaine WD 20 group electrophysiology - minimum stimulation protocol in 5 animals and rectification index in 5 animals by A.Suska.

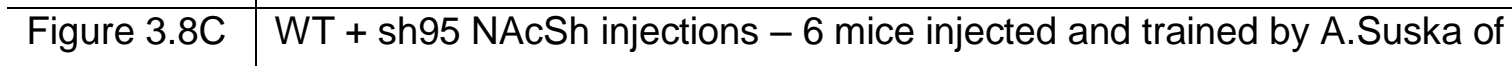
which 3 mice were kept for WD 20.

SAP102 KO + control virus NAcSh injection - 3 mice injected by A.Suska. SAP102 KO + sh95 NAcSh injection - 14 mice injected, out which 6 mice were trained till WD 1 and 2 mice till WD 20 by A. Suska

Figure 3.9G $\quad$ WT + sh95 WD 20 group electrophysiology - minimum stimulation protocol in 4 animals and rectification index in 3 animals by A.Suska.

SAP102 +sh95 WD 1 group electrophysiology - minimum stimulation protocol in 3 animals and rectification index in 3 animals by A.Suska.

SAP102 +sh95 WD 20 group electrophysiology - minimum stimulation protocol in 1 animal and rectification index in 1 animal by $A$.Suska. 


\section{References}

Abraham, W.C., and Bear, M.F. (1996). Metaplasticity: the plasticity of synaptic plasticity. Trends Neurosci. 19, 126-130.

Arendt, K.L., Royo, M., Fernández-Monreal, M., Knafo, S., Petrok, C.N., Martens, J.R., and Esteban, J.A. (2010). PIP3 controls synaptic function by maintaining AMPA receptor clustering at the postsynaptic membrane. Nat. Neurosci. 13, 36-44.

Barres, B.A. (2008). The Mystery and Magic of Glia: A Perspective on Their Roles in Health and Disease. Neuron 60, 430-440.

Beattie, E.C., Carroll, R.C., Yu, X., Morishita, W., Yasuda, H., von Zastrow, M., and Malenka, R.C. (2000). Regulation of AMPA receptor endocytosis by a signaling mechanism shared with LTD. Nat. Neurosci. 3, 1291-1300.

Béique, J.-C., Lin, D.-T., Kang, M.-G., Aizawa, H., Takamiya, K., and Huganir, R.L. (2006). Synapse-specific regulation of AMPA receptor function by PSD-95. Proc. Natl. Acad. Sci. 103, 19535-19540.

Belin, D., and Everitt, B.J. (2008). Cocaine Seeking Habits Depend upon DopamineDependent Serial Connectivity Linking the Ventral with the Dorsal Striatum. Neuron 57, 432441.

Bellone, C., and Lüscher, C. (2005). mGluRs induce a long-term depression in the ventral tegmental area that involves a switch of the subunit composition of AMPA receptors. Eur. J. Neurosci. 21, 1280-1288.

Bellone, C., and Lüscher, C. (2006). Cocaine triggered AMPA receptor redistribution is reversed in vivo by mGluR-dependent long-term depression. Nat. Neurosci. 9, 636-641.

Bellone, C., Lüscher, C., and Mameli, M. (2008). Mechanisms of synaptic depression triggered by metabotropic glutamate receptors. Cell. Mol. Life Sci. 65, 2913-2923.

Bennett, M.V.L., and Zukin, R.S. (2004). Electrical Coupling and Neuronal Synchronization in the Mammalian Brain. Neuron 41, 495-511.

Berridge, M.J. (1998). Neuronal Calcium Signaling. Neuron 21, 13-26.

Birnboim, H.C., and Doly, J. (1979). A rapid alkaline extraction procedure for screening recombinant plasmid DNA. Nucleic Acids Res. 7, 1513-1523.

Bliss, T.V.P., and Lømo, T. (1973). Long-lasting potentiation of synaptic transmission in the dentate area of the anaesthetized rabbit following stimulation of the perforant path. J. Physiol. 232, 331-356.

Boehm, J., and Malinow, R. (2005). AMPA receptor phosphorylation during synaptic plasticity. Biochem. Soc. Trans. 33, 1354-1356. 


\section{References}

Bonnet, S.A.D., Akad, D.S., Samaddar, T., Liu, Y., Huang, X., Dong, Y., and Schlüter, O.M. (2013). Synaptic State-Dependent Functional Interplay between Postsynaptic Density-95 and Synapse-Associated Protein 102. J. Neurosci. 33, 13398-13409.

Bouton, M.E. (2002). Context, ambiguity, and unlearning: sources of relapse after behavioral extinction. Biol. Psychiatry 52, 976-986.

Brown, T.E., Lee, B.R., Mu, P., Ferguson, D., Dietz, D., Ohnishi, Y.N., Lin, Y., Suska, A., Ishikawa, M., Huang, Y.H., et al. (2011). A Silent Synapse-Based Mechanism for CocaineInduced Locomotor Sensitization. J. Neurosci. 31, 8163-8174.

Burns, M.E., and Augustine, G.J. (1995). Synaptic structure and function: Dynamic organization yields architectural precision. Cell 83, 187-194.

Busetto, G., Higley, M.J., and Sabatini, B.L. (2008). Developmental presence and disappearance of postsynaptically silent synapses on dendritic spines of rat layer $2 / 3$ pyramidal neurons. J. Physiol. 586, 1519-1527.

Carroll, R.C., Beattie, E.C., Xia, H., Lüscher, C., Altschuler, Y., Nicoll, R.A., Malenka, R.C., and von Zastrow, M. (1999). Dynamin-dependent endocytosis of ionotropic glutamate receptors. Proc. Natl. Acad. Sci. U. S. A. 96, 14112-14117.

Carroll, R.C., Beattie, E.C., von Zastrow, M., and Malenka, R.C. (2001). Role of ampa receptor endocytosis in synaptic plasticity. Nat. Rev. Neurosci. 2, 315-324.

Cartmell, J., and Schoepp, D.D. (2000). Regulation of Neurotransmitter Release by Metabotropic Glutamate Receptors. J. Neurochem. 75, 889-907.

Catterall, W.A., and Few, A.P. (2008). Calcium Channel Regulation and Presynaptic Plasticity. Neuron 59, 882-901.

Chen, B.T., Bowers, M.S., Martin, M., Hopf, F.W., Guillory, A.M., Carelli, R.M., Chou, J.K., and Bonci, A. (2008). Cocaine but Not Natural Reward Self-Administration nor Passive Cocaine Infusion Produces Persistent LTP in the VTA. Neuron 59, 288-297.

Chen, L., Chetkovich, D.M., Petralia, R.S., Sweeney, N.T., Kawasaki, Y., Wenthold, R.J., Bredt, D.S., and Nicoll, R.A. (2000). Stargazin regulates synaptic targeting of AMPA receptors by two distinct mechanisms. Nature 408, 936-943.

Chen, R., Tilley, M.R., Wei, H., Zhou, F., Zhou, F.-M., Ching, S., Quan, N., Stephens, R.L., Hill, E.R., Nottoli, T., et al. (2006). Abolished cocaine reward in mice with a cocaineinsensitive dopamine transporter. Proc. Natl. Acad. Sci. 103, 9333-9338.

Cho, K.-O., Hunt, C.A., and Kennedy, M.B. (1992). The rat brain postsynaptic density fraction contains a homolog of the drosophila discs-large tumor suppressor protein. Neuron 9, 929942.

Christopherson, K.S., Ullian, E.M., Stokes, C.C.A., Mullowney, C.E., Hell, J.W., Agah, A., Lawler, J., Mosher, D.F., Bornstein, P., and Barres, B.A. (2005). Thrombospondins Are Astrocyte-Secreted Proteins that Promote CNS Synaptogenesis. Cell 120, 421-433. 
Di Ciano, P., Robbins, T.W., and Everitt, B.J. (2007). Differential Effects of Nucleus Accumbens Core, Shell, or Dorsal Striatal Inactivations on the Persistence, Reacquisition, or Reinstatement of Responding for a Drug-Paired Conditioned Reinforcer. Neuropsychopharmacology 33, 1413-1425.

Collingridge, G.L., Kehl, S.J., and McLennan, H. (1983). Excitatory amino acids in synaptic transmission in the Schaffer collateral-commissural pathway of the rat hippocampus. J. Physiol. 334, 33-46.

Collingridge, G.L., Olsen, R.W., Peters, J., and Spedding, M. (2009). A nomenclature for ligand-gated ion channels. Neuropharmacology 56, 2-5.

Conrad, K.L., Tseng, K.Y., Uejima, J.L., Reimers, J.M., Heng, L.-J., Shaham, Y., Marinelli, M., and Wolf, M.E. (2008). Formation of accumbens GluR2-lacking AMPA receptors mediates incubation of cocaine craving. Nature 454, 118-121.

Cruz, F.C., Babin, K.R., Leao, R.M., Goldart, E.M., Bossert, J.M., Shaham, Y., and Hope, B.T. (2014). Role of Nucleus Accumbens Shell Neuronal Ensembles in Context-Induced Reinstatement of Cocaine-Seeking. J. Neurosci. 34, 7437-7446.

Cunningham, C.L., Gremel, C.M., and Groblewski, P.A. (2006). Drug-induced conditioned place preference and aversion in mice. Nat. Protoc. 1, 1662-1670.

Cuthbert, P.C., Stanford, L.E., Coba, M.P., Ainge, J.A., Fink, A.E., Opazo, P., Delgado, J.Y., Komiyama, N.H., O'Dell, T.J., and Grant, S.G.N. (2007). Synapse-Associated Protein 102/dlgh3 Couples the NMDA Receptor to Specific Plasticity Pathways and Learning Strategies. J. Neurosci. 27, 2673-2682.

Dingledine, R., and Conn, P.J. (2000). Peripheral Glutamate Receptors: Molecular Biology and Role in Taste Sensation. J. Nutr. 130, 1039-1039.

Dingledine, R., Borges, K., Bowie, D., and Traynelis, S.F. (1999). The Glutamate Receptor Ion Channels. Pharmacol. Rev. 51, 7-62.

Dong, Y., and Nestler, E.J. (2014a). The neural rejuvenation hypothesis of cocaine addiction. Trends Pharmacol. Sci. 35, 374-383.

Dong, Y., and Nestler, E.J. (2014b). The neural rejuvenation hypothesis of cocaine addiction. Trends Pharmacol. Sci. 35, 374-383.

Dudek, S.M., and Bear, M.F. (1992). Homosynaptic long-term depression in area CA1 of hippocampus and effects of $\mathrm{N}$-methyl-D-aspartate receptor blockade. Proc. Natl. Acad. Sci. U. S. A. 89, 4363-4367.

Durand, G.M., Kovalchuk, Y., and Konnerth, A. (1996). Long-term potentiation and functional synapse induction in developing hippocampus. Nature $381,71-75$.

Edgar, J.M., and Nave, K.-A. (2009). The role of CNS glia in preserving axon function. Curr. Opin. Neurobiol. 19, 498-504. 


\section{References}

Ehrlich, I., and Malinow, R. (2004). Postsynaptic Density 95 controls AMPA Receptor Incorporation during Long-Term Potentiation and Experience-Driven Synaptic Plasticity. J. Neurosci. 24, 916-927.

El-Husseini, A.E.-D., Schnell, E., Chetkovich, D.M., Nicoll, R.A., and Bredt, D.S. (2000). PSD95 Involvement in Maturation of Excitatory Synapses. Science 290, 1364-1368.

Elias, G.M., Funke, L., Stein, V., Grant, S.G., Bredt, D.S., and Nicoll, R.A. (2006). SynapseSpecific and Developmentally Regulated Targeting of AMPA Receptors by a Family of MAGUK Scaffolding Proteins. Neuron 52, 307-320.

Eroglu, C. (2009). The role of astrocyte-secreted matricellular proteins in central nervous system development and function. J. Cell Commun. Signal. 3, 167-176.

Eroglu, Ç., Allen, N.J., Susman, M.W., O'Rourke, N.A., Park, C.Y., Özkan, E., Chakraborty, C., Mulinyawe, S.B., Annis, D.S., Huberman, A.D., et al. (2009). Gabapentin Receptor $\alpha 2 \delta-1$ Is a Neuronal Thrombospondin Receptor Responsible for Excitatory CNS Synaptogenesis. Cell 139, 380-392.

Evans, R.M., and Zamponi, G.W. (2006). Presynaptic Ca2+ channels - integration centers for neuronal signaling pathways. Trends Neurosci. 29, 617-624.

Everitt, B.J., and Robbins, T.W. (2005). Neural systems of reinforcement for drug addiction: from actions to habits to compulsion. Nat. Neurosci. 8, 1481-1489.

Feng, W., and Zhang, M. (2009). Organization and dynamics of PDZ-domain-related supramodules in the postsynaptic density. Nat. Rev. Neurosci. 10, 87-99.

French, S.J., and Totterdell, S. (2002). Hippocampal and prefrontal cortical inputs monosynaptically converge with individual projection neurons of the nucleus accumbens. J. Comp. Neurol. 446, 151-165.

French, S.J., and Totterdell, S. (2003). Individual nucleus accumbens-projection neurons receive both basolateral amygdala and ventral subicular afferents in rats. Neuroscience 119, 19-31.

Fuchs, R.A., Evans, K.A., Parker, M.C., and See, R.E. (2004). Differential involvement of the core and shell subregions of the nucleus accumbens in conditioned cue-induced reinstatement of cocaine seeking in rats. Psychopharmacology (Berl.) 176, 459-465.

Garner, C.C., Zhai, R.G., Gundelfinger, E.D., and Ziv, N.E. (2002). Molecular mechanisms of CNS synaptogenesis. Trends Neurosci. 25, 243-250.

Gawin FH, and Kleber HD (1986). Abstinence symptomatology and psychiatric diagnosis in cocaine abusers: Clinical observations. Arch. Gen. Psychiatry 43, 107-113.

Gee, N.S., Brown, J.P., Dissanayake, V.U.K., Offord, J., Thurlow, R., and Woodruff, G.N. (1996). The Novel Anticonvulsant Drug, Gabapentin (Neurontin), Binds to the Subunit of a Calcium Channel. J. Biol. Chem. 271, 5768-5776. 
Giese, K.P., Fedorov, N.B., Filipkowski, R.K., and Silva, A.J. (1998). Autophosphorylation at Thr286 of the a Calcium-Calmodulin Kinase II in LTP and Learning. Science 279, 870-873.

Goebel, D.J., and Poosch, M.S. (1999). NMDA receptor subunit gene expression in the rat brain: a quantitative analysis of endogenous mRNA levels of NR1Com, NR2A, NR2B, NR2C, NR2D and NR3A. Mol. Brain Res. 69, 164-170.

Golding, N.L., Staff, N.P., and Spruston, N. (2002). Dendritic spikes as a mechanism for cooperative long-term potentiation. Nature 418, 326-331.

Graham, F.L., Smiley, J., Russell, W.C., and Nairn, R. (1977). Characteristics of a human cell line transformed by DNA from human adenovirus type 5. J. Gen. Virol. 36, 59-74.

Granger, A.J., and Nicoll, R.A. (2014). LTD expression is independent of glutamate receptor subtype. Front. Synaptic Neurosci. 6.

Granger, A.J., Shi, Y., Lu, W., Cerpas, M., and Nicoll, R.A. (2013). LTP requires a reserve pool of glutamate receptors independent of subunit type. Nature 493, 495-500.

Gray, E.G. (1963). Electron microscopy of presynaptic organelles of the spinal cord. J. Anat. 97, 101-106.5.

Gray, J.A., Shi, Y., Usui, H., During, M.J., Sakimura, K., and Nicoll, R.A. (2011). Distinct Modes of AMPA Receptor Suppression at Developing Synapses by GluN2A and GluN2B: Single-Cell NMDA Receptor Subunit Deletion In Vivo. Neuron 71, 1085-1101.

Grimm, J.W., Hope, B.T., Wise, R.A., and Shaham, Y. (2001). Neuroadaptation: Incubation of cocaine craving after withdrawal. Nature 412, 141-142.

Groc, L., Gustafsson, B., and Hanse, E. (2006). AMPA signalling in nascent glutamatergic synapses: there and not there! Trends Neurosci. 29, 132-139.

Halbout, B., Bernardi, R.E., Hansson, A.C., and Spanagel, R. (2014). Incubation of Cocaine Seeking following Brief Cocaine Experience in Mice Is Enhanced by mGluR1 Blockade. J. Neurosci. 34, 1781-1790.

Hanse, E., Seth, H., and Riebe, I. (2013). AMPA-silent synapses in brain development and pathology. Nat. Rev. Neurosci. 14, 839-850.

Hollmann, M., and Heinemann, S. (1994). Cloned Glutamate Receptors. Annu. Rev. Neurosci. 17, 31-108.

Huang, X., Stodieck, S.K., Goetze, B., Cui, L., Wong, M.H., Wenzel, C., Hosang, L., Dong, Y., Löwel, S., and Schlüter, O.M. (2015a). Progressive maturation of silent synapses governs the duration of a critical period. Proc. Natl. Acad. Sci. U. S. A. 112, E3131-E3140.

Huang, Y.H., Lin, Y., Mu, P., Lee, B.R., Brown, T.E., Wayman, G., Marie, H., Liu, W., Yan, Z., Sorg, B.A., et al. (2009). In Vivo Cocaine Experience Generates Silent Synapses. Neuron 63, 40-47. 


\section{References}

Huang, Y.H., Schlüter, O.M., and Dong, Y. (2015b). Silent Synapses Speak Up Updates of the Neural Rejuvenation Hypothesis of Drug Addiction. The Neuroscientist 1073858415579405.

Hyman, S.E., and Malenka, R.C. (2001). Addiction and the brain: The neurobiology of compulsion and its persistence. Nat. Rev. Neurosci. 2, 695-703.

Hyman, S.E., Malenka, R.C., and Nestler, E.J. (2006a). NEURAL MECHANISMS OF ADDICTION: The Role of Reward-Related Learning and Memory. Annu. Rev. Neurosci. 29, 565-598.

Hyman, S.E., Malenka, R.C., and Nestler, E.J. (2006b). NEURAL MECHANISMS OF ADDICTION: The Role of Reward-Related Learning and Memory. Annu. Rev. Neurosci. 29, 565-598.

Isaac, J.T.R., Nicoll, R.A., and Malenka, R.C. (1995). Evidence for silent synapses: Implications for the expression of LTP. Neuron 15, 427-434.

Isaac, J.T.R., Crair, M.C., Nicoll, R.A., and Malenka, R.C. (1997). Silent Synapses during Development of Thalamocortical Inputs. Neuron 18, 269-280.

Ito, M. (1989). Long-Term Depression. Annu. Rev. Neurosci. 12, 85-102.

Ito, R., Dalley, J.W., Robbins, T.W., and Everitt, B.J. (2002). Dopamine Release in the Dorsal Striatum during Cocaine-Seeking Behavior under the Control of a Drug-Associated Cue. J. Neurosci. 22, 6247-6253.

Ito, R., Robbins, T.W., and Everitt, B.J. (2004). Differential control over cocaine-seeking behavior by nucleus accumbens core and shell. Nat. Neurosci. 7, 389-397.

Kalivas, P.W. (2004). Glutamate systems in cocaine addiction. Curr. Opin. Pharmacol. 4, 2329.

Kauer, J.A. (2004). Learning Mechanisms in Addiction: Synaptic Plasticity in the Ventral Tegmental Area as a Result of Exposure to Drugs of Abuse. Annu. Rev. Physiol. 66, 447475.

Kauer, J.A., and Malenka, R.C. (2007). Synaptic plasticity and addiction. Nat. Rev. Neurosci. 8, 844-858.

Kelley, A.E., and Berridge, K.C. (2002). The Neuroscience of Natural Rewards: Relevance to Addictive Drugs. J. Neurosci. 22, 3306-3311.

Kelly, L., Farrant, M., and Cull-Candy, S.G. (2009). Synaptic mGluR activation drives plasticity of calcium-permeable AMPA receptors. Nat. Neurosci. 12, 593-601.

Kerchner, G.A., and Nicoll, R.A. (2008). Silent synapses and the emergence of a postsynaptic mechanism for LTP. Nat. Rev. Neurosci. 9, 813.

Kim, E., and Sheng, M. (2004). PDZ domain proteins of synapses. Nat. Rev. Neurosci. 5, 771-781. 
Kistner, U., Wenzel, B.M., Veh, R.W., Cases-Langhoff, C., Garner, A.M., Appeltauer, U., Voss, B., Gundelfinger, E.D., and Garner, C.C. (1993). SAP90, a rat presynaptic protein related to the product of the Drosophila tumor suppressor gene dlg-A. J. Biol. Chem. 268, 4580-4583.

Kleckner, N.W., and Dingledine, R. (1988). Requirement for glycine in activation of NMDAreceptors expressed in Xenopus oocytes. Science 241, 835-837.

Koob, G.F., and Volkow, N.D. (2009). Neurocircuitry of Addiction. Neuropsychopharmacology 35, 217-238.

Kornau, H.C., Schenker, L.T., Kennedy, M.B., and Seeburg, P.H. (1995). Domain interaction between NMDA receptor subunits and the postsynaptic density protein PSD-95. Science 269, 1737-1740.

Kourrich, S., Rothwell, P.E., Klug, J.R., and Thomas, M.J. (2007). Cocaine Experience Controls Bidirectional Synaptic Plasticity in the Nucleus Accumbens. J. Neurosci. 27, 79217928.

Koya, E., Cruz, F.C., Ator, R., Golden, S.A., Hoffman, A.F., Lupica, C.R., and Hope, B.T. (2012). Silent synapses in selectively activated nucleus accumbens neurons following cocaine sensitization. Nat. Neurosci. 15, 1556-1562.

Kristensen, A.S., Jenkins, M.A., Banke, T.G., Schousboe, A., Makino, Y., Johnson, R.C., Huganir, R., and Traynelis, S.F. (2011). Mechanism of Ca2+/calmodulin-dependent kinase II regulation of AMPA receptor gating. Nat. Neurosci. 14, 727-735.

Kullmann, D.M. (1994). Amplitude fluctuations of. Neuron 12, 1111-1120.

Kuroda, H., Kutner, R.H., Bazan, N.G., and Reiser, J. (2009). Simplified lentivirus vector production in protein-free media using polyethylenimine-mediated transfection. J. Virol. Methods 157, 113-121.

Laube, B., Kuhse, J., and Betz, H. (1998). Evidence for a Tetrameric Structure of Recombinant NMDA Receptors. J. Neurosci. 18, 2954-2961.

Lee, B.R., and Dong, Y. (2011). Cocaine-induced metaplasticity in the nucleus accumbens: Silent synapse and beyond. Neuropharmacology 61, 1060-1069.

Lee, B.R., Ma, Y.-Y., Huang, Y.H., Wang, X., Otaka, M., Ishikawa, M., Neumann, P.A., Graziane, N.M., Brown, T.E., Suska, A., et al. (2013). Maturation of silent synapses in amygdala-accumbens projection contributes to incubation of cocaine craving. Nat. Neurosci. 16, 1644-1651.

Lee, H.-K., Kameyama, K., Huganir, R.L., and Bear, M.F. (1998). NMDA Induces Long-Term Synaptic Depression and Dephosphorylation of the GluR1 Subunit of AMPA Receptors in Hippocampus. Neuron 21, 1151-1162.

Lee, H.-K., Barbarosie, M., Kameyama, K., Bear, M.F., and Huganir, R.L. (2000). Regulation of distinct AMPA receptor phosphorylation sites during bidirectional synaptic plasticity. Nature 405, 955-959. 
Lerma, J. (2003). Roles and rules of kainate receptors in synaptic transmission. Nat. Rev. Neurosci. 4, 481-495.

Liao, D., Hessler, N.A., and Malinow, R. (1995). Activation of postsynaptically silent synapses during pairing-induced LTP in CA1 region of hippocampal slice. Nature 375, 400-404.

Lisman, J., and Raghavachari, S. (2006). A Unified Model of the Presynaptic and Postsynaptic Changes During LTP at CA1 Synapses. Sci. Signal. 2006, re11-re11.

Liu, S.J., and Zukin, R.S. (2007). Ca2+-permeable AMPA receptors in synaptic plasticity and neuronal death. Trends Neurosci. 30, 126-134.

Liu, X.-B., Murray, K.D., and Jones, E.G. (2004). Switching of NMDA Receptor 2A and 2B Subunits at Thalamic and Cortical Synapses during Early Postnatal Development. J. Neurosci. 24, 8885-8895.

Lois, C., Hong, E.J., Pease, S., Brown, E.J., and Baltimore, D. (2002). Germline Transmission and Tissue-Specific Expression of Transgenes Delivered by Lentiviral Vectors. Science 295, 868-872.

Loweth, J.A., Tseng, K.Y., and Wolf, M.E. (2013a). Using metabotropic glutamate receptors to modulate cocaine's synaptic and behavioral effects: mGluR1 finds a niche. Curr. Opin. Neurobiol. 23, 500-506.

Loweth, J.A., Tseng, K.Y., and Wolf, M.E. (2013b). Using metabotropic glutamate receptors to modulate cocaine's synaptic and behavioral effects: mGluR1 finds a niche. Curr. Opin. Neurobiol. 23, 500-506.

Loweth, J.A., Scheyer, A.F., Milovanovic, M., LaCrosse, A.L., Flores-Barrera, E., Werner, C.T., Li, X., Ford, K.A., Le, T., Olive, M.F., et al. (2014a). Synaptic depression via mGluR1 positive allosteric modulation suppresses cue-induced cocaine craving. Nat. Neurosci. 17, 73-80.

Loweth, J.A., Scheyer, A.F., Milovanovic, M., LaCrosse, A.L., Flores-Barrera, E., Werner, C.T., Li, X., Ford, K.A., Le, T., Olive, M.F., et al. (2014b). Synaptic depression via mGluR1 positive allosteric modulation suppresses cue-induced cocaine craving. Nat. Neurosci. 17, 73-80.

Lu, W., Shi, Y., Jackson, A.C., Bjorgan, K., During, M.J., Sprengel, R., Seeburg, P.H., and Nicoll, R.A. (2009). Subunit composition of synaptic AMPA receptors revealed by a single-cell genetic approach. Neuron 62, 254-268.

Lujan, R., Nusser, Z., Roberts, J.D., Shigemoto, R., and Somogyi, P. (1996). Perisynaptic location of metabotropic glutamate receptors mGluR1 and mGluR 5 on dendrites and dendritic spines in the rat hippocampus. Eur. J. Neurosci. 8, 1488-1500.

Lüscher, C., and Malenka, R.C. (2011). Drug-Evoked Synaptic Plasticity in Addiction: From Molecular Changes to Circuit Remodeling. Neuron 69, 650-663. 
Lynch, W.J., Nicholson, K.L., Dance, M.E., Morgan, R.W., and Foley, P.L. (2010). Animal Models of Substance Abuse and Addiction: Implications for Science, Animal Welfare, and Society. Comp. Med. 60, 177-188.

Ma, Y.-Y., Lee, B.R., Wang, X., Guo, C., Liu, L., Cui, R., Lan, Y., Balcita-Pedicino, J.J., Wolf, M.E., Sesack, S.R., et al. (2014). Bidirectional Modulation of Incubation of Cocaine Craving by Silent Synapse-Based Remodeling of Prefrontal Cortex to Accumbens Projections. Neuron 83, 1453-1467.

Magee, J.C., and Johnston, D. (1997). A Synaptically Controlled, Associative Signal for Hebbian Plasticity in Hippocampal Neurons. Science 275, 209-213.

Mameli, M., Balland, B., Luján, R., and Lüscher, C. (2007). Rapid Synthesis and Synaptic Insertion of GluR2 for mGluR-LTD in the Ventral Tegmental Area. Science 317, 530-533.

Mameli, M., Halbout, B., Creton, C., Engblom, D., Parkitna, J.R., Spanagel, R., and Lüscher, C. (2009). Cocaine-evoked synaptic plasticity: persistence in the VTA triggers adaptations in the NAc. Nat. Neurosci. 12, 1036-1041.

Mansour, M., Nagarajan, N., Nehring, R.B., Clements, J.D., and Rosenmund, C. (2001). Heteromeric AMPA Receptors Assemble with a Preferred Subunit Stoichiometry and Spatial Arrangement. Neuron 32, 841-853.

Masters, J.R., and Stacey, G.N. (2007). Changing medium and passaging cell lines. Nat. Protoc. 2, 2276-2284.

Matsuzaki, M., Honkura, N., Ellis-Davies, G.C.R., and Kasai, H. (2004). Structural basis of long-term potentiation in single dendritic spines. Nature 429, 761-766.

McCutcheon, J.E., Wang, X., Tseng, K.Y., Wolf, M.E., and Marinelli, M. (2011a). CalciumPermeable AMPA Receptors Are Present in Nucleus Accumbens Synapses after Prolonged Withdrawal from Cocaine Self-Administration But Not Experimenter-Administered Cocaine. J. Neurosci. 31, 5737-5743.

McCutcheon, J.E., Loweth, J.A., Ford, K.A., Marinelli, M., Wolf, M.E., and Tseng, K.Y. (2011b). Group I mGluR Activation Reverses Cocaine-Induced Accumulation of CalciumPermeable AMPA Receptors in Nucleus Accumbens Synapses via a Protein Kinase CDependent Mechanism. J. Neurosci. 31, 14536-14541.

McGee, A.W., Topinka, J.R., Hashimoto, K., Petralia, R.S., Kakizawa, S., Kauer, F., AguileraMoreno, A., Wenthold, R.J., Kano, M., and Bredt, D.S. (2001). PSD-93 Knock-Out Mice Reveal That Neuronal MAGUKs Are Not Required for Development or Function of Parallel Fiber Synapses in Cerebellum. J. Neurosci. 21, 3085-3091.

McLellan A, Lewis DC, O'Brien CP, and Kleber HD (2000). Drug dependence, a chronic medical illness: Implications for treatment, insurance, and outcomes evaluation. JAMA 284, 1689-1695.

Merrill, E.G., and Wall, P.D. (1972). Factors forming the edge of a receptive field: the presence of relatively ineffective afferent terminals. J. Physiol. 226, 825-846. 
Meyer-Franke, A., Kaplan, M.R., Pfieger, F.W., and Barres, B.A. (1995). Characterization of the signaling interactions that promote the survival and growth of developing retinal ganglion cells in culture. Neuron 15, 805-819.

Migaud, M., Charlesworth, P., Dempster, M., Webster, L.C., Watabe, A.M., Makhinson, M., He, Y., Ramsay, M.F., Morris, R.G.M., Morrison, J.H., et al. (1998). Enhanced long-term potentiation and impaired learning in mice with mutant postsynaptic density-95 protein. Nature 396, 433-439.

Ann M. Lohof, N.D.-B. (1996). Synapse Elimination in the Central Nervous System: Functional Significance and Cellular Mechanisms. Rev. Neurosci. 7, 85-101.

Mogenson, G.J., Jones, D.L., and Yim, C.Y. (1980). From motivation to action: Functional interface between the limbic system and the motor system. Prog. Neurobiol. 14, 69-97.

Montague, P.R., Hyman, S.E., and Cohen, J.D. (2004). Computational roles for dopamine in behavioural control. Nature 431, 760-767.

Mu, P., Moyer, J.T., Ishikawa, M., Zhang, Y., Panksepp, J., Sorg, B.A., Schlüter, O.M., and Dong, Y. (2010). Exposure to Cocaine Dynamically Regulates the Intrinsic Membrane Excitability of Nucleus Accumbens Neurons. J. Neurosci. Off. J. Soc. Neurosci. 30, 36893699.

Mueller, D., and Stewart, J. (2000). Cocaine-induced conditioned place preference: reinstatement by priming injections of cocaine after extinction. Behav. Brain Res. 115, 39-47.

Mulkey, R.M., and Malenka, R.C. (1992). Mechanisms underlying induction of homosynaptic long-term depression in area CA1 of the hippocampus. Neuron 9, 967-975.

Mulkey, R.M., Herron, C.E., and Malenka, R.C. (1993). An essential role for protein phosphatases in hippocampal long-term depression. Science 261, 1051-1055.

Mulkey, R.M., Endo, S., Shenolikar, S., and Malenka, R.C. (1994). Involvement of a calcineurin/ inhibitor-1 phosphatase cascade in hippocampal long-term depression. Nature 369, 486-488.

Ngomba, R.T., Santolini, I., Biagioni, F., Molinaro, G., Simonyi, A., van Rijn, C.M., D'Amore, V., Mastroiacovo, F., Olivieri, G., Gradini, R., et al. (2011). Protective role for type-1 metabotropic glutamate receptors against spike and wave discharges in the WAG/Rij rat model of absence epilepsy. Neuropharmacology 60, 1281-1291.

Nicola, S.M. (2010). The Flexible Approach Hypothesis: Unification of Effort and CueResponding Hypotheses for the Role of Nucleus Accumbens Dopamine in the Activation of Reward-Seeking Behavior. J. Neurosci. 30, 16585-16600.

Nicoll, R.A., and Roche, K.W. (2013). Long-term potentiation: peeling the onion. Neuropharmacology 74, 18-22.

Nicoll, R.A., Malenka, R.C., and Kauer, J.A. (1990). Functional comparison of neurotransmitter receptor subtypes in mammalian central nervous system. Physiol. Rev. 70, 513-565. 
Niv, Y., Daw, N.D., Joel, D., and Dayan, P. (2006). Tonic dopamine: opportunity costs and the control of response vigor. Psychopharmacology (Berl.) 191, 507-520.

Nusser, Z., Lujan, R., Laube, G., Roberts, J.D.B., Molnar, E., and Somogyi, P. (1998). Cell Type and Pathway Dependence of Synaptic AMPA Receptor Number and Variability in the Hippocampus. Neuron 21, 545-559.

O'brien, C.P., Childress, A.R., McLELLAN, A.T., and Ehrman, R. (1992). Classical Conditioning in Drug-Dependent Humansa. Ann. N. Y. Acad. Sci. 654, 400-415.

Oh, M.C., Derkach, V.A., Guire, E.S., and Soderling, T.R. (2006). Extrasynaptic Membrane Trafficking Regulated by GluR1 Serine 845 Phosphorylation Primes AMPA Receptors for Long-term Potentiation. J. Biol. Chem. 281, 752-758.

Opazo, P., and Choquet, D. (2011). A three-step model for the synaptic recruitment of AMPA receptors. Mol. Cell. Neurosci. 46, 1-8.

Opazo, P., Sainlos, M., and Choquet, D. (2012). Regulation of AMPA receptor surface diffusion by PSD-95 slots. Curr. Opin. Neurobiol. 22, 453-460.

Palay, S.L. (1956). Synapses in the Central Nervous System. J. Biophys. Biochem. Cytol. 2, 193-202.

Paoletti, P. (2011). Molecular basis of NMDA receptor functional diversity. Eur. J. Neurosci. 33, 1351-1365.

Papp, E., Borhegyi, Z., Tomioka, R., Rockland, K.S., Mody, I., and Freund, T.F. (2011). Glutamatergic input from specific sources influences the nucleus accumbens-ventral pallidum information flow. Brain Struct. Funct. 217, 37-48.

Pascoli, V., Terrier, J., Espallergues, J., Valjent, E., O'Connor, E.C., and Lüscher, C. (2014). Contrasting forms of cocaine-evoked plasticity control components of relapse. Nature 509, 459-464.

Peng, J., Kim, M.J., Cheng, D., Duong, D.M., Gygi, S.P., and Sheng, M. (2004). Semiquantitative Proteomic Analysis of Rat Forebrain Postsynaptic Density Fractions by Mass Spectrometry. J. Biol. Chem. 279, 21003-21011.

Petralia, R.S., Esteban, J.A., Wang, Y.-X., Partridge, J.G., Zhao, H.-M., Wenthold, R.J., and Malinow, R. (1999). Selective acquisition of AMPA receptors over postnatal development suggests a molecular basis for silent synapses. Nat. Neurosci. 2, 31-36.

Pettit, D.L., Perlman, S., and Malinow, R. (1994). Potentiated transmission and prevention of further LTP by increased CaMKII activity in postsynaptic hippocampal slice neurons. Science $266,1881-1885$.

Pfrieger, F.W., and Barres, B.A. (1997). Synaptic Efficacy Enhanced by Glial Cells in Vitro. Science 277, 1684-1687.

Pickens, C.L., Airavaara, M., Theberge, F., Fanous, S., Hope, B.T., and Shaham, Y. (2011). Neurobiology of the incubation of drug craving. Trends Neurosci. 34, 411-420. 
Pomierny-Chamioło, L., Rup, K., Pomierny, B., Niedzielska, E., Kalivas, P.W., and Filip, M. (2014). Metabotropic glutamatergic receptors and their ligands in drug addiction. Pharmacol. Ther. 142, 281-305.

Pontieri, F.E., Tanda, G., and Di Chiara, G. (1995). Intravenous cocaine, morphine, and amphetamine preferentially increase extracellular dopamine in the "shell" as compared with the "core" of the rat nucleus accumbens. Proc. Natl. Acad. Sci. U. S. A. 92, 12304-12308.

Procko, C., and Shaham, S. (2009). Synaptogenesis: New Roles for an Old Player. Curr. Biol. 19, R1114-R1115.

Quinlan, E.M., Olstein, D.H., and Bear, M.F. (1999). Bidirectional, experience-dependent regulation of $\mathrm{N}$-methyl-d-aspartate receptor subunit composition in the rat visual cortex during postnatal development. Proc. Natl. Acad. Sci. U. S. A. 96, 12876-12880.

Reid, C.A., Bekkers, J.M., and Clements, J.D. (2003). Presynaptic Ca2+ channels: a functional patchwork. Trends Neurosci. 26, 683-687.

Rekling, J.C., Shao, X.M., and Feldman, J.L. (2000). Electrical coupling and excitatory synaptic transmission between rhythmogenic respiratory neurons in the preBötzinger complex. J. Neurosci. Off. J. Soc. Neurosci. 20, RC113.

Richmond, S.A., Irving, A.J., Molnár, E., Mcllhinney, R.A.J., Michelangeli, F., Henley, J.M., and Collingridge, G.L. (1996). Localization of the glutamate receptor subunit GluR1 on the surface of living and within cultured hippocampal neurons1. Neuroscience 75, 69-82.

Risher, W.C., and Eroglu, C. (2012). Thrombospondins as key regulators of synaptogenesis in the central nervous system. Matrix Biol. 31, 170-177.

Ritz, M.C., Lamb, R.J., Goldberg, S.R., and Kuhar, M.J. (1987). Cocaine receptors on dopamine transporters are related to self-administration of cocaine. Science 237, 1219-1223.

Robbe, D., Kopf, M., Remaury, A., Bockaert, J., and Manzoni, O.J. (2002). Endogenous cannabinoids mediate long-term synaptic depression in the nucleus accumbens. Proc. Natl. Acad. Sci. 99, 8384-8388.

Robbins, T.W., and Everitt, B.J. (1996a). Neurobehavioural mechanisms of reward and motivation. Curr. Opin. Neurobiol. 6, 228-236.

Robbins, T.W., and Everitt, B.J. (1996b). Neurobehavioural mechanisms of reward and motivation. Curr. Opin. Neurobiol. 6, 228-236.

Robinson, T.E., and Kolb, B. (1999). Alterations in the morphology of dendrites and dendritic spines in the nucleus accumbens and prefrontal cortex following repeated treatment with amphetamine or cocaine. Eur. J. Neurosci. 11, 1598-1604.

Robinson, T.E., and Kolb, B. (2004). Structural plasticity associated with exposure to drugs of abuse. Neuropharmacology 47, Supplement 1, 33-46. 
Robinson, T.E., Gorny, G., Mitton, E., and Kolb, B. (2001). Cocaine self-administration alters the morphology of dendrites and dendritic spines in the nucleus accumbens and neocortex. Synap. N. Y. N 39, 257-266.

Roche, K.W., Standley, S., McCallum, J., Dune Ly, C., Ehlers, M.D., and Wenthold, R.J. (2001). Molecular determinants of NMDA receptor internalization. Nat. Neurosci. 4, 794-802.

Rodd-Henricks, Z.A., McKinzie, D.L., Li, T.-K., Murphy, J.M., and McBride, W.J. (2002). Cocaine Is Self-Administered into the Shell but Not the Core of the Nucleus Accumbens of Wistar Rats. J. Pharmacol. Exp. Ther. 303, 1216-1226.

Roux, S., Froger, C., Porsolt, R.D., Valverde, O., and Maldonado, R. (2003). Place preference test in rodents. Curr. Protoc. Neurosci. Editor. Board Jacqueline N Crawley Al Chapter 9, Unit 9.15.

Saal, D., Dong, Y., Bonci, A., and Malenka, R.C. (2003). Drugs of Abuse and Stress Trigger a Common Synaptic Adaptation in Dopamine Neurons. Neuron 37, 577-582.

Saiki, R.K., Scharf, S., Faloona, F., Mullis, K.B., Horn, G.T., Erlich, H.A., and Arnheim, N. (1985). Enzymatic amplification of beta-globin genomic sequences and restriction site analysis for diagnosis of sickle cell anemia. Science 230, 1350-1354.

Sanchis-Segura, C., and Spanagel, R. (2006). REVIEW: Behavioural assessment of drug reinforcement and addictive features in rodents: an overview. Addict. Biol. 11, 2-38.

Saunders, B.T., and Robinson, T.E. (2012). The role of dopamine in the accumbens core in the expression of Pavlovian-conditioned responses. Eur. J. Neurosci. 36, 2521-2532.

Scannevin, R.H., and Huganir, R.L. (2000). Postsynaptic organisation and regulation of excitatory synapses. Nat. Rev. Neurosci. 1, 133-141.

Schlüter, O.M., Xu, W., and Malenka, R.C. (2006). Alternative N-Terminal Domains of PSD95 and SAP97 Govern Activity-Dependent Regulation of Synaptic AMPA Receptor Function. Neuron 51, 99-111.

Schnell, E., Sizemore, M., Karimzadegan, S., Chen, L., Bredt, D.S., and Nicoll, R.A. (2002). Direct interactions between PSD-95 and stargazin control synaptic AMPA receptor number. Proc. Natl. Acad. Sci. U. S. A. 99, 13902-13907.

Schultz, W. (2011). Potential Vulnerabilities of Neuronal Reward, Risk, and Decision Mechanisms to Addictive Drugs. Neuron 69, 603-617.

Seeburg, P.H., Higuchi, M., and Sprengel, R. (1998). RNA editing of brain glutamate receptor channels: mechanism and physiology1. Brain Res. Rev. 26, 217-229.

Self, D.W., Choi, K.-H., Simmons, D., Walker, J.R., and Smagula, C.S. (2004). Extinction Training Regulates Neuroadaptive Responses to Withdrawal from Chronic Cocaine SelfAdministration. Learn. Mem. 11, 648-657.

Sheng, M. (2001a). Molecular organization of the postsynaptic specialization. Proc. Natl. Acad. Sci. 98, 7058-7061. 
Sheng, M. (2001b). Molecular organization of the postsynaptic specialization. Proc. Natl. Acad. Sci. U. S. A. 98, 7058-7061.

Sheng, M., and Hoogenraad, C.C. (2007). The Postsynaptic Architecture of Excitatory Synapses: A More Quantitative View. Annu. Rev. Biochem. 76, 823-847.

Sheng, M., Cummings, J., Roldan, L.A., Jan, Y.N., and Jan, L.Y. (1994). Changing subunit composition of heteromeric NMDA receptors during development of rat cortex. Nature 368 , 144-147.

Shepherd, J.D., and Huganir, R.L. (2007). The Cell Biology of Synaptic Plasticity: AMPA Receptor Trafficking. Annu. Rev. Cell Dev. Biol. 23, 613-643.

Shi, S.-H., Hayashi, Y., Esteban, J.A., and Malinow, R. (2001). Subunit-Specific Rules Governing AMPA Receptor Trafficking to Synapses in Hippocampal Pyramidal Neurons. Cell 105, 331-343.

Spencer, S., Brown, R.M., Quintero, G.C., Kupchik, Y.M., Thomas, C.A., Reissner, K.J., and Kalivas, P.W. (2014). $\alpha 2 \delta-1$ Signaling in Nucleus Accumbens Is Necessary for CocaineInduced Relapse. J. Neurosci. 34, 8605-8611.

Stefanik, M.T., and Kalivas, P.W. (2013). Optogenetic dissection of basolateral amygdala projections during cue-induced reinstatement of cocaine seeking. Front. Behav. Neurosci. 7, 213.

Stefanik, M.T., Kupchik, Y.M., and Kalivas, P.W. (2015). Optogenetic inhibition of cortical afferents in the nucleus accumbens simultaneously prevents cue-induced transient synaptic potentiation and cocaine-seeking behavior. Brain Struct. Funct. 1-9.

Stein, V., House, D.R.C., Bredt, D.S., and Nicoll, R.A. (2003). Postsynaptic Density-95 Mimics and Occludes Hippocampal Long-Term Potentiation and Enhances Long-Term Depression. J. Neurosci. 23, 5503-5506.

Stern, P., Edwards, F.A., and Sakmann, B. (1992). Fast and slow components of unitary EPSCs on stellate cells elicited by focal stimulation in slices of rat visual cortex. J. Physiol. 449, 247-278.

Straub, C., and Tomita, S. (2012). The regulation of glutamate receptor trafficking and function by TARPs and other transmembrane auxiliary subunits. Curr. Opin. Neurobiol. 22, 488-495.

Streit, W.J. (2002). Microglia as neuroprotective, immunocompetent cells of the CNS. Glia 40, 133-139.

Sudhof, T.C. (2004). The Synaptic Vesicle Cycle. Annu. Rev. Neurosci. 27, 509-547.

Tardin, C., Cognet, L., Bats, C., Lounis, B., and Choquet, D. (2003). Direct imaging of lateral movements of AMPA receptors inside synapses. EMBO J. 22, 4656-4665. 
Thomas, M.J., Beurrier, C., Bonci, A., and Malenka, R.C. (2001). Long-term depression in the nucleus accumbens: a neural correlate of behavioral sensitization to cocaine. Nat. Neurosci. 4, 1217-1223.

Tomita, S., Stein, V., Stocker, T.J., Nicoll, R.A., and Bredt, D.S. (2005). Bidirectional Synaptic Plasticity Regulated by Phosphorylation of Stargazin-like TARPs. Neuron 45, 269-277.

Tong, G., Takahashi, H., Tu, S., Shin, Y., Talantova, M., Zago, W., Xia, P., Nie, Z., Goetz, T., Zhang, D., et al. (2008). Modulation of NMDA Receptor Properties and Synaptic Transmission by the NR3A Subunit in Mouse Hippocampal and Cerebrocortical Neurons. J. Neurophysiol. 99, 122-132.

Traynelis, S.F., Wollmuth, L.P., McBain, C.J., Menniti, F.S., Vance, K.M., Ogden, K.K., Hansen, K.B., Yuan, H., Myers, S.J., and Dingledine, R. (2010). Glutamate Receptor Ion Channels: Structure, Regulation, and Function. Pharmacol. Rev. 62, 405-496.

Ullian, E.M., Sapperstein, S.K., Christopherson, K.S., and Barres, B.A. (2001). Control of Synapse Number by Glia. Science 291, 657-661.

Ullian, E.M., Christopherson, K.S., and Barres, B.A. (2004). Role for glia in synaptogenesis. Glia 47, 209-216.

Ungless, M.A., Whistler, J.L., Malenka, R.C., and Bonci, A. (2001). Single cocaine exposure in vivo induces long-term potentiation in dopamine neurons. Nature 411, 583-587.

Vanderschuren, L.J.M.J., Ciano, P.D., and Everitt, B.J. (2005). Involvement of the Dorsal Striatum in Cue-Controlled Cocaine Seeking. J. Neurosci. 25, 8665-8670.

Volterra, A., and Meldolesi, J. (2005). Astrocytes, from brain glue to communication elements: the revolution continues. Nat. Rev. Neurosci. 6, 626-640.

Wang, Y.T., and Linden, D.J. (2000). Expression of Cerebellar Long-Term Depression Requires Postsynaptic Clathrin-Mediated Endocytosis. Neuron 25, 635-647.

Wenthold, R.J., Petralia, R.S., J, I.B., and Niedzielski, A.S. (1996). Evidence for multiple AMPA receptor complexes in hippocampal CA1/CA2 neurons. J. Neurosci. 16, 1982-1989.

Whitaker, L.R., Carneiro de Oliveira, P.E., McPherson, K.B., Fallon, R.V., Planeta, C.S., Bonci, A., and Hope, B.T. Associative Learning Drives the Formation of Silent Synapses in Neuronal Ensembles of the Nucleus Accumbens. Biol. Psychiatry.

White, F.J. (1996). Synaptic Regulation of Mesocorticolimbic Dopamine Neurons. Annu. Rev. Neurosci. 19, 405-436.

Whitlock, J.R., Heynen, A.J., Shuler, M.G., and Bear, M.F. (2006). Learning Induces LongTerm Potentiation in the Hippocampus. Science 313, 1093-1097.

Wojcik, S.M., and Brose, N. (2007). Regulation of Membrane Fusion in Synaptic ExcitationSecretion Coupling: Speed and Accuracy Matter. Neuron 55, 11-24. 


\section{References}

Wolf, M.E. (2010). The Bermuda Triangle of cocaine-induced neuroadaptations. Trends Neurosci. 33, 391-398.

Wolf, M.E., and Tseng, K.-Y. (2012). Calcium-permeable AMPA receptors in the VTA and nucleus accumbens after cocaine exposure: when, how, and why? Front. Mol. Neurosci. 5, 72.

Wu, G., Malinow, R., and Cline, H.T. (1996). Maturation of a central glutamatergic synapse. Science 274, 972-976.

Xiao, M.-Y., Wasling, P., Hanse, E., and Gustafsson, B. (2004). Creation of AMPA-silent synapses in the neonatal hippocampus. Nat. Neurosci. 7, 236-243.

Xu, J., Xiao, N., and Xia, J. (2010). Thrombospondin 1 accelerates synaptogenesis in hippocampal neurons through neuroligin 1. Nat. Neurosci. 13, 22-24.

Xu, W., Schlüter, O.M., Steiner, P., Czervionke, B.L., Sabatini, B., and Malenka, R.C. (2008). Molecular Dissociation of the Role of PSD-95 in Regulating Synaptic Strength and LTD. Neuron 57, 248-262.

Yao, W.-D., Gainetdinov, R.R., Arbuckle, M.I., Sotnikova, T.D., Cyr, M., Beaulieu, J.-M., Torres, G.E., Grant, S.G.N., and Caron, M.G. (2004). Identification of PSD-95 as a Regulator of Dopamine-Mediated Synaptic and Behavioral Plasticity. Neuron 41, 625-638.

Yin, H.H., Knowlton, B.J., and Balleine, B.W. (2004). Lesions of dorsolateral striatum preserve outcome expectancy but disrupt habit formation in instrumental learning. Eur. J. Neurosci. 19, 181-189.

Yuste, R., and Bonhoeffer, T. (2001). Morphological Changes in Dendritic Spines Associated with Long-Term Synaptic Plasticity. Annu. Rev. Neurosci. 24, 1071-1089.

Zhu, J.J., Esteban, J.A., Hayashi, Y., and Malinow, R. (2000). Postnatal synaptic potentiation: Delivery of GluR4-containing AMPA receptors by spontaneous activity. Nat. Neurosci. 3, 1098-1106. 


\section{Acknowledgements}

I would like to express my heartfelt gratitude towards all those who have motivated, mentored and assisted me during the course of my PhD.

Firstly I would like to sincerely thank, my supervisor Dr. Dr. Oliver Schlüter for his guidance and support throughout my PhD. I have learnt a lot from his scientific rigor and determination. I will forever be grateful for the patience and understanding he has shown towards me.

The IMPRS Molecular Biology Program office and the GGNB office have always been prompt and supportive with every big and small issue, which was a huge help during my stay in Göttingen. I would like to extend a special thanks to the IMPRS Molecular Biology program coordinator Dr. Steffen Burkhardt who made it possible for me to transition from molecular biology to neuroscience rather swiftly and smoothly. His active support enabled me to make it through many rough patches.

I thank my core thesis committee members - Prof. Tobias Moser and Prof. Silvio Rizzoli for their valuable input and encouragement throughout my PhD. I am also very grateful to our collaborator Prof. Yan Dong who gave me the chance to learn cutting edge scientific techniques at his lab in Pittsburgh, PA, USA.

I am greatly indebted to all present and former members of the Molecular Neurobiology Laboratory at ENI-Göttingen for their kind cooperation and advice. The technical (and emotional) support provided by Sandra Ott-Gebauer, I'm sure was the best I could ever receive. I have benefited immensely from Sandra's help and understanding. During my initial days as a PhD, I learnt a lot from Dr. Anna Suska, for which I am very grateful. I have benefited a lot from the presence of all the lab members - Lei, Derya, Huang, Plinio, Yanling, Joanna, Man Ho, Peter, Lisa and Tanmoy, who were all responsible for creating a friendly working environment. I was fortunate to be in a lab where everyone was always willing to offer a friendly word of advice. I am thankful to Myrto who during her master thesis, and my last months of $\mathrm{PhD}$, assisted me with many experiments and cheered up the atmosphere with her positive outlook. I would also like to thank students whom I supervised for a short period Kanishka and Monika, for their contribution in my findings.

My experience at ENI was significantly bettered by members of other research groups who shared the floor with us. Vinita and Ankit were always there to provide compassion, motivation and entertainment of many diverse forms. It was truly wonderful that they were available for a good laugh, a not-so-quick bite, a lengthy word of advice on life and of course 
scientific discussion as well. Markus, Plinio and Charlie also provided good company in and out of lab. The enthusiastic mingling of labs on my institute floor made for a healthy and joyful work environment that I will always cherish.

During my PhD, I am glad to have maintained my friendship with Ishita and Anisha, my closest friends from school, who in spite of living far away for many years, are just as close to me as before. However, I could not have managed my PhD studies without the unending support of my friends in Göttingen. I was lucky to have several Indian friends in Germany who served as my core support group. For me, the apartments of Veena, Vinita, Kundan, Heena, Ankit and Pawan collectively served as a second home. The many evenings spent together with them, the dinners, the movies, the potluck get-togethers, the birthday celebrations, the bollywood dancing and singing and even the argumentative debates will always remain as one of my fondest memories. I thank them all for being there for me always and for making me feel comfortable while confiding in them. I had the good fortune of spending time with more friends like Sumir, Priyanka, Upasana, Ananya, Soham and Arnab who all made for great company during fun times. I also found a confidant in Annie, whose insight helped me sail through some rough waters. Jatin and Mayur are my friends who often took up a mentoring role when it was required, for which I am very thankful. My classmates and other friends namely Metin, Sona, Jonas, Ingrid, Maria and Ömer also offered friendly advice whenever it was sought from them. Meeting up with them occasionally was always very pleasant, as discussions with them broadened my perspective on work and life. My friends have not just been helpful but also inspirational, as I have learnt from them how to tackle various problems in personal and professional life. Making good friends during tough times, I believe is one of the best by-products of my PhD studies.

I thank my fiancé Kunal, the one and only person who has actually lived and witnessed my $\mathrm{PhD}$ period most closely. I consider myself to have a strong pillar of support in him, by my side through all these years. I thank him for listening to my incessant whining and complaining whether it made logical sense or not, and whether he understood it or not. I also thank him for bearing with me, the nerdy and geeky partner speaking non-stop sentences full of scientific jargon. My happiest and also most productive days were those spent with his company in Germany, howsoever few or infrequent they were. He has motivated me throughout all trying and testing times, believed in me when I was at my lowest and also inspired me by his own passion, courage, hard work and self-discipline. I feel I owe this academic achievement to Kunal who helped me make it my personal achievement. 
Last but not the least, my family back in India has been more than patient and supportive towards my PhD. I am blessed to have a family that can never be too far away from me in mind and soul, as I derive mental strength from them. My mother's empathy, father's encouragement and sister's pragmatism has kept me going, even when I thought I was too worn out. Though their understanding of my field of work was very limited, they always did their very best to support me. My family has enabled me to come this far and will continue to motivate me to strive for excellence. 
8. Curriculum Vitae 


\section{Curriculum Vitae}

\section{Personal data}

First name, last name

Date of birth

Avani Shukla

Place of birth

3rd October 1989

Address

New Delhi, India

Email

Ginsterweg 10, Göttingen, 37077, Germany

a.shukla@eni-g.de,shukla.avani@gmail.com

\section{Education}

04/2012 - 05/2016 PhD studies in Molecular Neurobiology

With Dr. Dr. Oliver M. Schlüter

European Neuroscience Institute - Göttingen, Germany

PhD Thesis: "Cocaine induced synaptic plasticity in the nucleus

accumbens and drug-asociated behavior: An unexpected dissociation"

Collaborative research done at Department of Neuroscience, University

of Pittsburgh, PA, USA with Prof. Yan Dong: "Deciphering the nucleus accumbens- specific role of PSD-95 in incubation of cocaine craving"

\section{9/2010 - 03/2012 M.Sc studies in Molecular Biology}

International Max Planck research School, Göttingen, Germany

M.Sc thesis with Prof. Nils Brose and Dr. Jeong-Seop Rhee at Max

Planck Institute for Experimental Medicine, Göttingen Germany:

"Functional analysis of the cell adhesion molecule IgSF11 in primary neuron culture"

\section{7/2007 - 06/2010 B.Sc (Honors) in Biochemistry}

Sri Venkateswara College, University of Delhi, India

3-term summer internship project with Prof. M.R.S. Rao at Jawaharlal

Nehru Centre for Advanced Scientific research, Bengaluru, India:

"Identification of the site of acetylation and methylation on a testis-

specific protein involved in mammalian spermiogenesis" 


\section{Academic awards and scholarships}

2011 - 2012 Awarded GGNB Excellence Stipend by Göttingen Graduate School for

Neurosciences and Molecular Biosciences (GGNB) for PhD project.

2010 - 2011 Secured top rank (grade A, perfect score of 1.0) in the Masters examination of IMPRS Molecular Biology Program, Göttingen, Germany.

Awarded Scholarship of the Excellence Foundation for the Promotion of the Max Planck Society.

2008 - 2010 Awarded a Diploma in Biology by Jawaharlal Nehru Centre for Advanced Scientific Research (JNCASR), Bangalore upon successful completion of Project Oriented Biological Education (POBE) a three-term summer training program for 10 B.Sc students selected from India.

2008 Secured $3^{\text {rd }}$ rank at University of Delhi for B.Sc (Honors) Biochemistry.

\section{Publications and presentations}

Publications "Cocaine-induced synaptic plasticity in the nucleus accumbens and drugassociated behaviour: An unexpected dissociation"

Shukla, A*., Suska A*., Olive F., Olive F., Loweth J., Dong Y. and Schlüter

OM.

European Neuroscience Institute - Göttingen, Germany

(In preparation)

* Authors contributed equally to the work

"Astrocyte-mediated induction of silent synapses upon cocaine exposure" Wang J., Shukla A., Schlüter OM and Dong Y.

University of Pittsburgh, USA and European Neuroscience Institute -

Göttingen, Germany

(In preparation) 
Poster "Fate of silent synapses in cocaine addiction"

presentations Shukla A., Suska A. and Schlüter OM.

$9^{\text {th }}$ FENS forum of Neuroscience, Milan, Italy - 2014

"Fate of silent synapses in cocaine addiction"

Shukla A., Suska A. and Schlüter OM.

Science day of Göttingen Graduate School for Neurosciences and Molecular Biosciences (GGNB), Göttingen, Germany -2013

"Analysis of compartment specific and cell autonomous loss-of-function of MeCP2"

Shukla A. and Schlüter OM

Tenth Göttingen Meeting of the German Neuroscience Society, Göttingen, Germany - 2013

Conference "Fate of silent synapses in cocaine addiction"

talks Graduate student workshop in Molecular Neuroscience, Boğaziçi University Istanbul, Turkey -2014 